(C) Copyright 2016

Chrissie Lim 


\title{
Alternative splicing takes charge of interleukin-22 and interferon lambda 4 signaling
}

\author{
Chrissie Lim
}

\begin{abstract}
A dissertation
submitted in partial fulfillment of the

requirements for the degree of
\end{abstract}

Doctor of Philosophy

University of Washington

2016

Reading Committee:

Dr. Ram Savan, Chair

Dr. Edward A. Clark

Dr. Mohamed Oukka

Program Authorized to Offer Degree:

Immunology 


\title{
University of Washington
}

\begin{abstract}
Chrissie Lim

Chair of the Supervisory Committee:

Assistant Professor, Dr. Ram Savan

Department of Immunology
\end{abstract}

Alternative splicing takes charge of interleukin-22 and interferon lambda 4 signaling

Immune responses require the tight control of dose, location, strength and duration through genetic, epigenetic or biochemical regulation. Of these, the generation of alternatively-spliced constructs increases transcriptional and proteomic diversity in post-transcriptional modification, localization and functional domain integrity. Specifically, this thesis explores how splice variation engenders profound differences in the biological functions of interleukin-22 (IL-22) binding protein (IL-22BP) and interferon lambda 4 (IFN $\lambda 4$ ), which are both central components of distinct cytokine pathways in mucosal immunity and inflammation. IL-22BP is a soluble receptor for IL-22 that is expressed as three isoforms in humans, though the physiological relevance of the three human isoforms has remained a mystery due to the absence of this variation in mice. We present novel findings that IL-22BPi1 is inactive due to intracellular retention by its unique exon, while IL-22BPi3 is also an antagonist but with differential activity from IL-22BPi2. Importantly, while IL-22BPi3 has widespread expression in steady-state homeostatic conditions, IL-22BPi2 is the only isoform induced by inflammatory TLR2/retinoic acid stimulation, highlighting important spatiotemporal control of the two isoforms that exploit their differential activities. IFN $\lambda 4$ presents a different mystery in which the protein-coding 
variant is genetically associated with poorer clearance, but the mechanism for this association remains unclear. We investigated several non-canonical functions proposed by the field, including intrinsic differences in activity of the three protein isoforms and their interference with antiviral activites of other type I or III interferons. Establishing an overexpression system and purifying recombinant proteins, we found that only the full-length isoform is active and exhibits similar effects to canonical type III IFN IFN $\lambda 3$, without any blockade of other IFN signaling. Simultaneously, functional IFN $\lambda 4$ expression is suppressed in hepatocytes and dendritic cells through preferential splicing to increase intron retention and expression of inactive isoforms. Therefore, alternative splicing in IFN $\lambda 4$ is an important mechanism to control IFN $\lambda 4$ bioactivity. The divergent manners in which alternative splice forms impact the activity of both IL-22BP and IFN $\lambda 4$ highlight the important contributions of this process to cytokine biology and bigger implications that escape detection by genomic analyses. 


\section{TABLE OF CONTENTS}

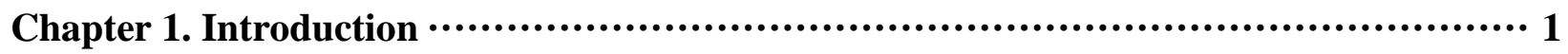

1.1 Regulation of protein expression and isoform by alternative splicing $\ldots \ldots \ldots \ldots \ldots \ldots \ldots 2$

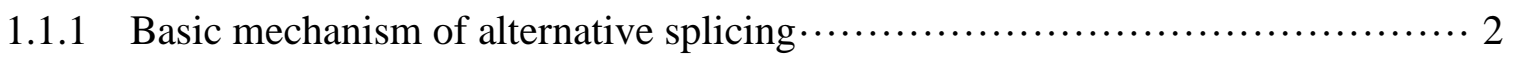

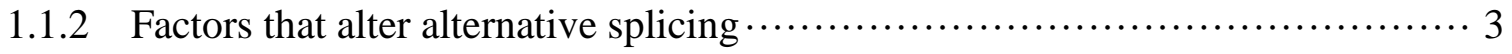

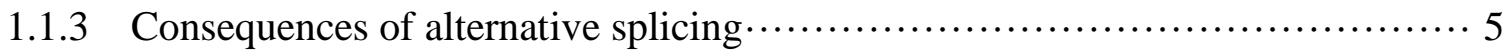

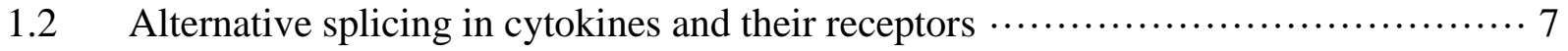

1.2.1 Splicing of IL-6 and its receptor diversifies signaling and regulatory modalities $\cdot 7$

1.2.2 Splicing of IL-15 and its receptor controls stability and secretion $. \ldots \ldots \ldots \ldots \ldots . . . .9$

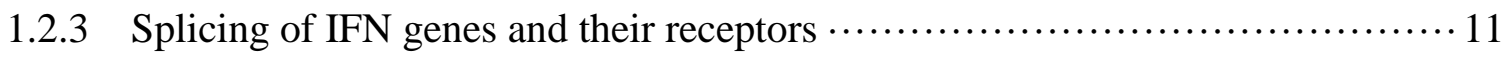

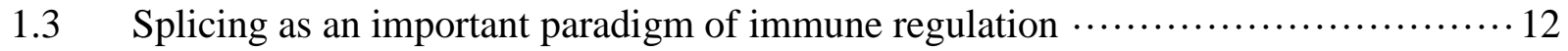

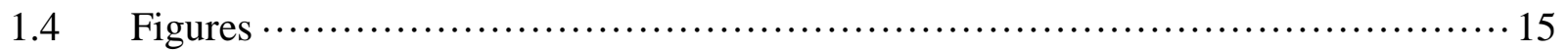

Chapter 2. The human IL-22 binding protein isoforms are a rheostat for IL-22 signaling. 20

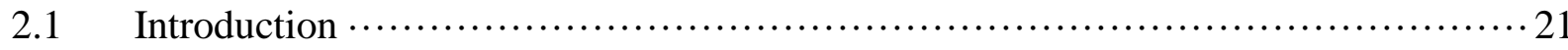

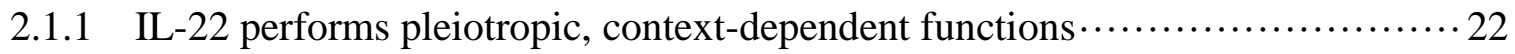

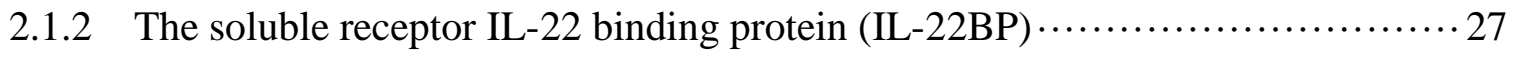

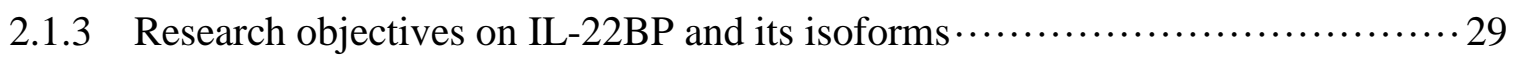

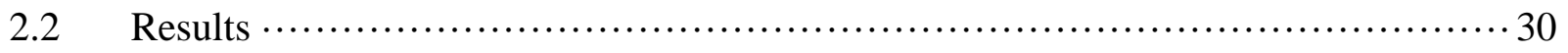

2.2.1 IL-22BP isoforms exhibit distinct activities against IL-22 signaling. $\cdots \ldots \ldots \ldots . \cdots 30$

2.2.2 Inactive IL-22BPi1 is retained intracellularly due to its third exon. $\cdots \cdots \ldots \ldots \ldots . \cdots 31$

2.2.3 Functional IL-22BP isoforms differentially modulate IL-22 activity............. 31

2.2.4 IL-22BPi2 and IL-22BPi3 differentially skew the IL-17/IL-22 axis.............. 32

2.2.5 IL22RA2v3 is the widely expressed isoform during homeostasis. $\cdots \ldots \ldots \ldots \ldots . \ldots 33$

2.2.6 IL22RA2v2 is the only isoform induced by the TLR2-retinoic acid pathway in

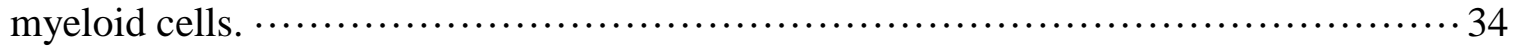

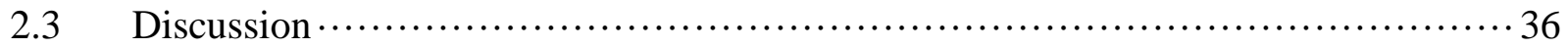

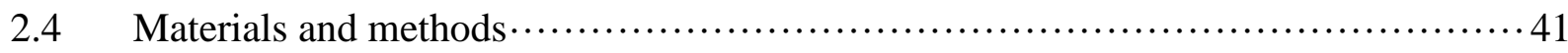

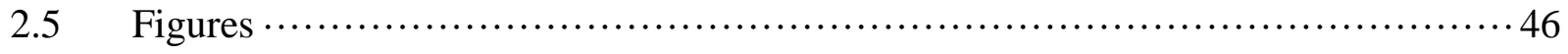


Chapter 3. Evolutionary adaptations suppress interferon lambda 4 expression during viral

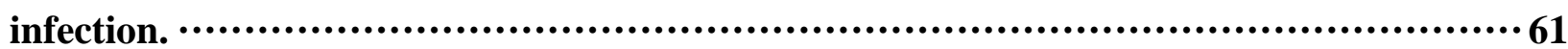

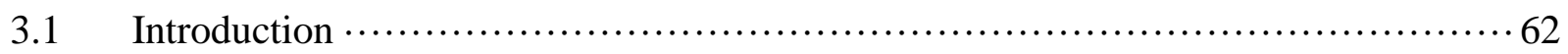

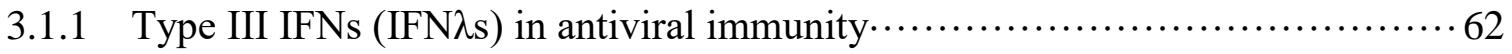

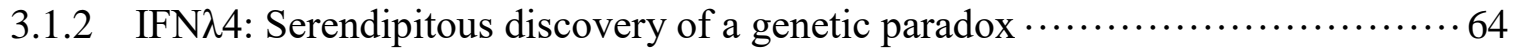

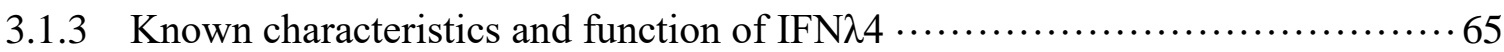

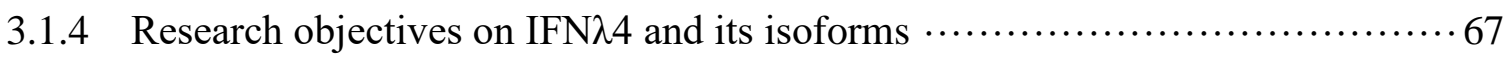

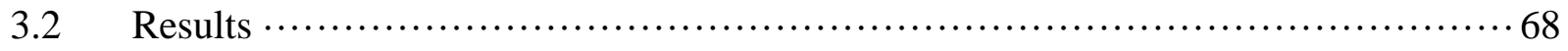

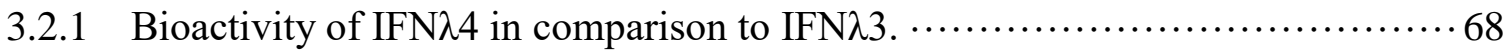

3.2.2 IFN $\lambda 4$ signals exclusively through the extracellular IFN $\lambda$ R1:IL-10R2 receptor

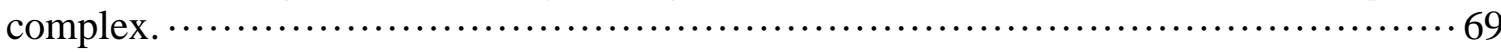

3.2.3 Intracellular IFN $\lambda 4$ isoforms do not affect type I and III IFN signaling. $\cdots \cdots \cdots \cdot 70$

3.2.4 Overexpressed IFN $\lambda 4$ p 179 and IFN $\lambda 3$ have comparable antiviral activity on HCV 71

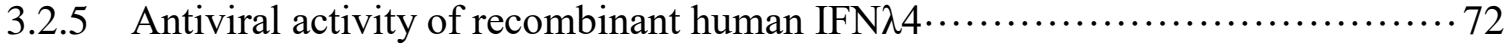

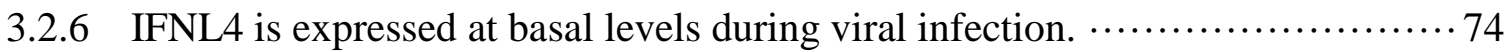

3.2.7 Endogenous IFNL4 isoforms are not translated efficiently during infection $\cdots \cdots 75$

3.2.8 Non-functional IFNL4 splice variants arose before the $\Delta \mathrm{G}>\mathrm{TT}$ frame-shift variant

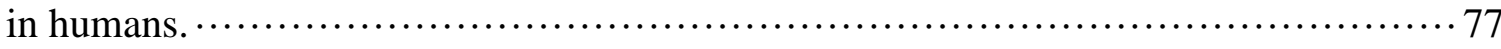

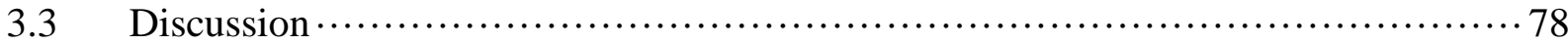

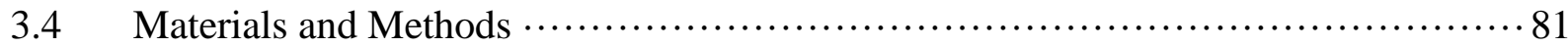

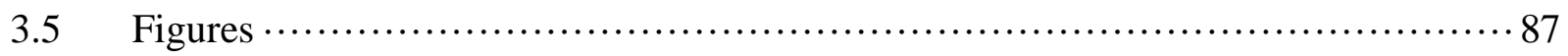

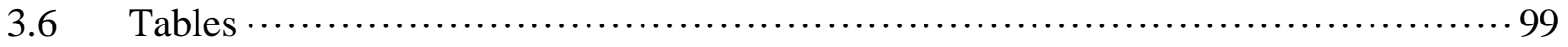

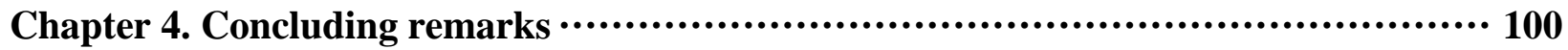

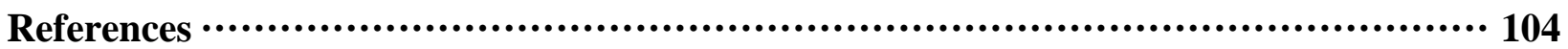




\section{LIST OF FIGURES}

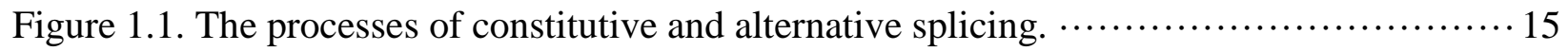

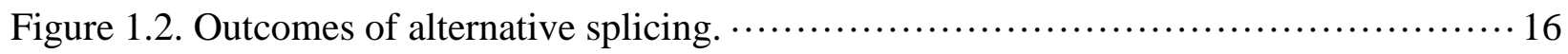

Figure 1.3. Alternative splicing of the IL-6 receptor increases diversity in IL-6 signaling. ...... 17

Figure 1.4. Alternative splicing of IL-15 changes its localization and post-translational

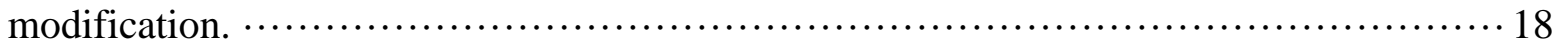

Figure 1.4. Alternative splicing of IL-15R $\alpha$ generates a soluble receptor that stabilizes IL-15. .19

Figure 2.1. Sources of IL-22 and its principal downstream signaling pathways. .............. 47

Figure 2.2. Known characteristics of IL-22 binding protein (IL-22BP) isoforms. .............. 46

Figure 2.1. IL-22BP isoforms differentially modulate IL-22 activity when overexpressed. …. 48

Figure 2.2. Recombinant human IL-22BPi2 and IL-22BPi3 proteins modulate IL-22 activity. . 50

Figure 2.3. IL22RA2v3 is the most widely expressed isoform, while IL22RA2v2 is the principal

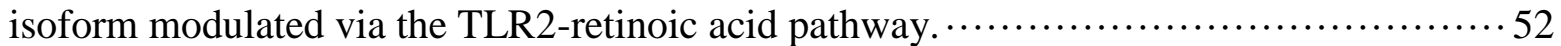

Supplementary Figure 2.1. Alignment of IL-22BPi1, IL-22BPi2 and IL-22BPi3 protein

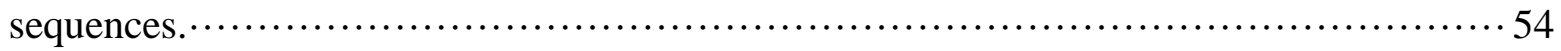

Supplementary Figure 2.2. Expression of IL-22BP isoforms in phospho-STAT3 immunoblot assays and optimization of dose for STAT3 luciferase reporter activity. $\cdots \cdots \cdots \cdots \cdots \cdots \cdots \cdots$

Supplementary Figure 2.3. Efficiency and specificity of the IL22RA2 Taqman probes. ……56

Supplementary Figure 2.4. Correlation between expression levels of IL-22 receptor and

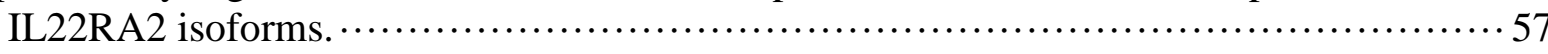

Supplementary Figure 2.5. IL22RA2 and ALDH1A2 expression in HL-60-derived neutrophils,

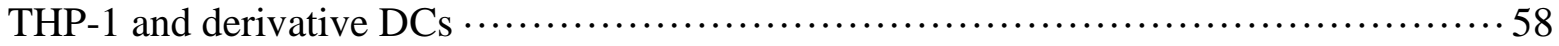

Supplementary Figure 2.6. Effects of IL-22BPi2 and IL-22BPi3 on IL-22 ELISA. ……......5 58

Supplementary Figure 2.7. IL-22BPi2 and IL-22BPi3 collaborate to form a rheostat that tunes

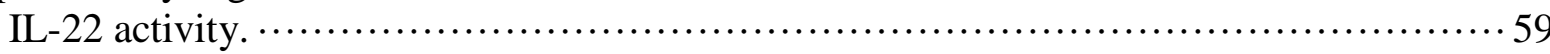

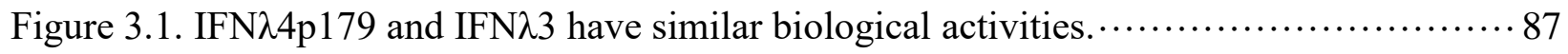

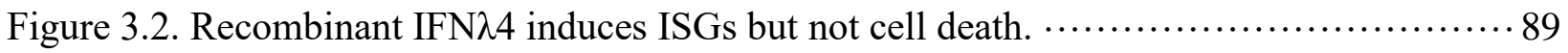

Figure 3.3. Recombinant human IFN $\lambda 4$ shows similar antiviral activity to IFN $\lambda 3 . \cdots \ldots \ldots \ldots \ldots 1$

Figure 3.4. Low IFNL4 induction during PAMP stimulation and viral infection. ...............92

Figure 3.5. IFNL4 mRNA are poorly loaded onto polyribosomes for translation and suppressed

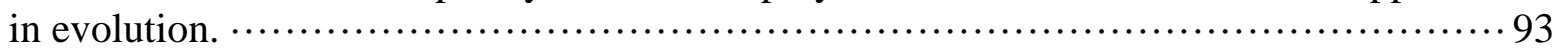

Figure 3.6. IFN $\lambda 4$ expression is suppressed during viral infections. ........................ 94

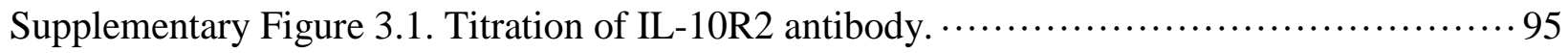

Supplementary Figure 3.2. Generation and characterization of $I F N L R 1^{-/-}$hepatocytes. ..........96 
Supplementary Figure 3.3. Sequence alignment of intron-retaining IFNL4 isoforms identified from a cDNA library from HepG2 cells treated with poly(I:C). ….................... 97

Supplementary Figure 3.4. Alignment of 3' UTR sequences of human IFNL1, IFNL2 and IFNL3

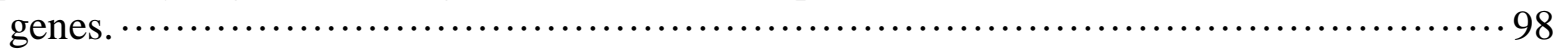

\section{LIST OF TABLES}

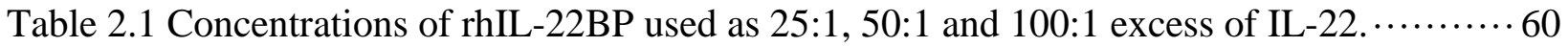

Table 2.2. Sequences of the qPCR probes and primers used to quantify IL22RA2 isoforms. $\cdots \cdots 6$

Table 3.1. Sequences of primers and probes used to clone, detect and genotype IFNL4. …....99 


\section{ACKNOWLEDGEMENTS}

I owe immense gratitude to my mentor Ram Savan for taking me on, in extreme faith, as one of his first two graduate students. He has gone out of his way to provide me with opportunities to succeed and has given me unwavering support in the tough endeavors of graduate school. Thanks also to him, I am a very fortunate member of a fun-loving lab, past and present, that is always ready to assist me in matters big and small, yet nonetheless pushes me hard to grow in science. In particular, Adelle P. McFarland provided inestimable help in setting up my project on interleukin-22 signaling and was always there with a smile throughout my graduate career even after starting her own.

The completion of my dissertation would not have been possible without the kindness, patience and generosity of many members of the biomedical community in Seattle. My thesis committee comprising Mohamed Oukka, Edward A. Clark and Joshua J. Woodward have provided invaluable input on the projects and made them a work I am truly proud of. Guidance and resources for protein purification were kindly provided by Marion Pepper and her trainees Akshay Krishamurty and Karen Kim, Joshua J. Woodward, Abigail Dietrich, Rachel Werther and Jazmine P. Hallinan. Members of Edward A. Clark's and Michael Gale Jr.'s labs have provided many satisfying scientific discussions and often rescued me during experimental emergencies. Last but not least, my graduate student community at UW Immunology, especially my classmates, have provided a beautifully welcoming and supportive environment that made coping with graduate school in a new country so much easier. Thank you, my Seattle family, for being the best home away from home I could ask for. 


\section{DEDICATION}




\section{Chapter 1. INTRODUCTION}

Generating alternative isoforms of a single protein or gene is a major mechanism by which the complexity of the proteome is magnified in multicellular eukaryotes. Isoforms can be created at the transcript level via alternative splicing of pre-messenger RNA (mRNA)s or the use of alternative transcription start sites. This process can be regulated post-transcriptionally by noncoding RNAs that bind to splice sites and create steric hindrance or alter the secondary structure of pre-mRNA that prevents binding by spliceosomal factors. Lastly, at the post-translational level, truncations can be generated by proteolytic cleavage that often change protein localization or function. Naturally, these processes that alter a genetic program's passage through the central dogma have profound effects in all central physiological processes including but not limited to development, homeostasis, immunity and regeneration. While exciting new technologies now enable us to profile splicing events on a more global, unbiased scale, it is still often impossible to predict the biological significance of alternative protein isoforms without targeted experimental validation.

A major interest of the Ram Lab is the control and function of cytokines, protein messengers in the immune system that coordinate immune responses against infection, and how they are controlled at the transcriptional and post-transcriptional levels to suit inflammatory context and kinetics. In this thesis, I investigate how the generation of multiple isoforms of cytokine or receptor is used to regulate two distinct signaling pathways involved in mucosal immunity and viral infection. 


\subsection{REGULATION OF PROTEIN EXPRESSION AND ISOFORM BY ALTERNATIVE SPLICING}

\subsubsection{Basic mechanism of alternative splicing}

It is estimated that $95 \%$ of multi-exon genes in humans undergo alternative splicing, and that 100,000 intermediate-to-high abundance programs alternative splicing events in major human tissues ${ }^{1}$. The core process of alternative splicing has been long established and details well covered in numerous reviews ${ }^{2-4}$; a brief summary of the process is presented below.

The process of pre-mRNA splicing process depends on a hierarchy of multiple elements. At its essential foundation, consensus splice sites at intron-exon boundaries are identified by the five small nuclear ribonuclear proteins (snRNPs): U1, U2 and the U4-U5-U6 tri-snRNP complex. These form the spliceosomal macromolecular assembly together with several auxiliary factors including U2AF65 and U2AF35m. The exon-intron junctions are recognized by snRNPs at the 5' "donor" GU site in the upstream exon and the 3' "acceptor" site at the AG in the downstream exon, usually guided by a polypyrimidine tract just upstream of the $3^{\prime}$ site. After activation by RNAdependent helicases and ATPases, the spliceosome performs the two transesterification reactions: the first to generate the lariat at a branch point adenosine within an intron which allows its excision, and the second to ligate the two adjacent exons (Fig. 1.1).

Each exon is included only if there is the spliceosome is able to recognize and bind the splice sites. Splice sites vary in strength for this selection: "strong" sites are fully used and lead to constitutive splicing, while choice of "weak" sites leads to alternative splicing, i.e. the generation of multiple, distinct mature mRNAs from a single pre-mRNA sequence. This is partially dependent on the similarity or divergence of the splice sites themselves from consensus sequences. The likelihood of exon usage is adjusted further by intronic or exonic splice enhancer (ISE, ESE) and 
suppressor (ISS, ESS) sequences lie close to the splice junctions and recruit proteins and ribonucleoproteins that in turn interact with the spliceosomal complexes on the splice sites (Fig. 1.1B). The most common trans-acting factors associated with this alternative splicing process are the Ser/Arg-rich protein families (SR) and heterogeneous nuclear ribonucleoproteisn (hnRNPs), although tissue-specific factors such as the FOX and neuro-oncological ventral antigen (NOVA) proteins may also participate. Together, these elements guide the spliceosomal protein machinery to the appropriate splice junctions required, preventing their random binding to cryptic splice sites.

\subsubsection{Factors that alter alternative splicing}

About $80 \%$ of splicing variability falls within the open reading frame, while the remaining occur in untranslated regions and affect cis-regulating elements ${ }^{3}$. Several mutations in exon 12 of the well-studied gene Cystic Fibrosis Transmembrane conductance Regulator (CFTR) result in drastic changes of exon inclusion down to $15 \%{ }^{5}$. While the effects of changes in splice junction sequences can be difficult to predict due to the promiscuity of surrounding enhancer motifs, it is known that some mutations in intronic variants can increase exon skipping, for example of exon 22 in $B R C A 2$ [REF]. In addition, deep intronic variants can generate new splice sites and therefore, pseudoexons; for instance, a variant in intron 20 of ataxia-telangectasia mutated (ATM) more than $0.5 \mathrm{~kb}$ from the nearest exons disrupts an intron-splicing processing element and creates an aberrant transcript with a cryptic exon ${ }^{6}$.

Interestingly, while exonic mutations on open reading frame are usually expected to alter protein function solely by nonsense or missense effects, synonymous mutations CFTR have been shown to change splicing efficiency of exons 9-12 as they alter 3' splice site and ESE sequences

to promote exon skipping ${ }^{7,8}$. Several supposedly neutral or synonymous mutations of ATM and 
neurofibromin-1 (NF1) also change their isoform expression. It is likely that many genomic variants that affect splicing in other genes remain unaccounted for.

Alternative splicing can also be altered by changes in trans-acting elements required for normal splicing which have broader effects on multiple genes. More than 150 proteins have been identified to be trans-acting factors in splicing, including kinases, phosphatases, helicases, mRNA transport factors and transcription factors ${ }^{3}$. While some of these factors are universal, such as the SR proteins, many have heterogeneous tissue expression that impact the exon usage of each single gene in multiple sites. For example, in activated murine macrophages, splicing during activation by cytokine stimulation or infection have been shown to be highly correlated with activation of additional myeloid factors including POU, CREBP and CEBP factors ${ }^{9}$. Meanwhile, many exon configurations are unique to the placental tissues and highly correlated with the expression of splicing factors ESRP and MBNL3 in the amnion and decidua, respectively ${ }^{10}$. Thanks to the co-occurrence of transcription, there is interaction between the spliceosome and transcription factors, initiation factors, elongation factors, RNA polymerase II and modified nucleosomes that determine exon inclusion or exclusion ${ }^{2}$. More recently, it has been recognized that non-coding RNAs including microRNAs and long non-coding RNAs can regulate binding of spliceosome machinery. In addition, secondary structure of RNA can also affect splicing by steric hindrance that alters accessibility to the spliceosomal complex ${ }^{11}$.

A final caveat remains in the use of animal models to study alternative splicing. Changes in alternative exon usage evolve quickly, with much larger and quicker transitions between species than gene expression largely due to changes in cis-acting elements ${ }^{12}$. In support of this, 6-8\% of exons are spliced differently in human and chimpanzee frontal cortex and heart tissue, despite the two species having $99 \%$ genomic identity ${ }^{13}$. This presents a major challenge to endogenous 
expression and regulation in vivo that also applies to our study of IL-22 binding protein and interferon lambda 4, as the isoforms of interest are both absent in popular animal models.

\subsubsection{Consequences of alternative splicing}

Alternative splicing of a transcript can occur in several modes including (1) inclusion of alternative cassette exons; (2) inclusion of mutually exclusive exons; (3) retention of introns leading to nonsense-mediated decay (NMD) and (4) use of alternative $5^{\prime}$ or $3^{\prime}$ splice sites (Fig. 1.2).

Choice of splice site affects both transcriptional and translational regulation, as well as the inclusion of functional domains in the resultant protein. For example, many soluble cytokine and growth factor receptors are generated from the same genes as membrane-bound receptors simply by exclusion of exons encoding the transmembrane domains. Often, this generates a soluble receptor that competitively blocks signaling by the membrane bound receptor from the same gene, as in the case of interleukin-1 (IL-1) receptor accessory protein (IL-1RacP) for IL-1 ${ }^{14}$. Immediately downstream of the cytokine receptor, the proximal mediator Myeloid differentiation primary response gene $88(\mathrm{MyD} 88)$ also undergoes splicing to generate a shorter isoform that excludes exon 2 that prevents activation of the next downstream inflammatory mediator, the innate signal transducer IL-1 receptor-associated kinase 1 (IRAK1), but preserves activation of the distinct pathway driven by c-Jun N-terminal kinase (JNK) and activator protein (AP-1 ${ }^{15-17}$. In turn, IRAK-1 naturally undergoes alternative splicing in humans to disrupt kinase activity of two isoforms and NFKB-dependent cytokine production without changing their association with signaling cofactors ${ }^{18,19}$. Alternative splicing in MyD88, IRAK-1 and other links of the chain including the Toll-like receptors, tumor necrosis factor receptor associated (TRAF) proteins and inhibitor of $\kappa \mathrm{B}$ kinase (IKK) subtly offer the immune system many opportunities to modulate the 
magnitude of activation of the inflammatory transcription factor nuclear factor $\kappa \mathrm{B}(\mathrm{NF} \kappa \mathrm{B})-$ all through the selective disruption of functional domains through alternative splice site and exon usage.

Excluding intron retention, about $1 / 3$ of all alternative splicing events lead to premature stop codons that cause mRNA degradation by nonsense mediated decay ${ }^{20}$. While NMD can function as a surveillance process that detects and eliminates the misfolded products of aberrant splicing, it is increasingly recognized as a targeted, efficient process coupled with alternative splicing to quickly and tightly regulate gene expression in many developmental and physiological pathways $^{21,22}$. For example, NMD is used to reduce expression of specific transcripts in adult tissue, while during development the exons containing premature stop codons are skipped to increase expression ${ }^{23}$.

Naturally, alteration of splicing events often have impacts on disease and the establishment of immunity. It is estimated that $60 \%$ of deleterious mutations cause disease by changing splicing patterns ${ }^{24}$. Mutations in snRNP assembly complexes give rise to neural diseases such as spinal muscular atrophy (SMA) and retinitis pigmentosa, caused by mutations in survival of motor neuron-1 (SMN-1) and pre-mRNA-processing factor gene homologues PRPF31, PRPF8 and HPRP3 respectively ${ }^{3}$. Changes in alternative splicing also have a major hand in alterations of important survival, antiapoptotic, metastatic and metabolic pathways that drive cancer progression, such as the tumor suppressor genes BRCAl and adenomatous polyposis coli $(A P C)^{25,26}$. In innate immunity, extensive alternative splicing of the Toll-like receptors (TLRs) generates new variants that change signaling strength or act as antagonists to the full-length protein, keeping runaway inflammatory signals in check $^{27}$. Alternative splicing also naturally abounds in adaptive immunity, which uses multiple methods to generate diversity required to 
detect an ever growing list of pathogens. Notably, alternative splicing of major histocompatibility complex molecules and other proteins in the antigen presentation pathway, such as the TAP1/2 and tapasin required to load peptides onto them, greatly increase the complexity of antigen presentation $^{28}$. Further downstream, the strong genetic association of IRF5 with systemic erythematosus lupus (SLE) implicates haplotypes of specific exon usage and splice sites that are likely to alter conveyance of interferon signaling. Despite the continuing challenges of parsing out a splicing code to accurately predict exon usage and splicing patterns, it remains a paramount consideration as a potential effect of RNA modifications and genomic variation.

\subsection{ALTERNATIVE SPLICING IN CYTOKINES AND THEIR RECEPTORS}

Many of the interleukins between IL-1 and IL-32 have known alternative isoforms that, when expressed, exhibit altered biological activity ${ }^{29}$. In addition, their receptors also undergo extensive alternative splicing to yield multiple forms that either compete for the ligand to suppress functional signaling, or otherwise synergize with the membrane-bound receptor to enhance downstream signaling ${ }^{29}$. Of this extensive list, I will expand on two specific examples below that demonstrate the profound and complex effects of splicing of cytokines and their receptors on the nature and strength of signaling. They are summarized in Fig. 1.3-1.5.

\subsubsection{Splicing of IL-6 and its receptor diversifies signaling and regulatory modalities}

An example of a cytokine signaling pathway heavily subject to regulation by alternative isoform usage is the inflammatory cytokine IL-6. It is expressed by many cells including activated monocytes, T cells and fibroblasts. IL-6 binds to its specific receptor IL-6R and the common family receptor chain gp130, to activating principally STAT3 but also NFKB and PI3K pathways ${ }^{30}$. IL-6 itself has several splice variants in both human and mouse: isoforms of human IL-6 lacking 
exons 2 and 4 have been identified in lung tissue; exclusion of exon 2 completely prevents translation of the protein, while IL-6 lacking exon 4 competitively inhibits full-length IL-6 binding to IL-6R and thus suppresses functional IL-6 signaling ${ }^{31}$.

In addition, both chains of the IL-6 receptor have classical membrane-bound forms as well as soluble forms (sIL-6R and sgp130) that can either be generated by alternative splicing or limited proteolysis of the membrane bound receptors by the proteases a disintegrin and $\underline{\text { metalloproteinase }}$ (ADAM)10 ADAM10 and ADAM17 ${ }^{32-35}$. Soluble IL-6R is able to bind IL-6 with high affinity and signal on cells expressing only gp130 in a process known as trans-signaling, in contrast to classical signaling that is mediated by IL-6 binding to mIL-6R and gp130. This process has several important implications that diversifies the biological functions and regulatory mechanisms of IL6 signaling. Firstly, while mIL-6R expression is limited to hepatocytes, megakaryocytes, neutrophils and some T cells, gp130 is expressed on all cells; therefore, IL-6 trans-signaling can occur on several populations that do lack mIL-6R, including human smooth muscle cells, neural cells, the endothelium and embryonic stem cells. Furthermore, the gene expression signatures of classical and trans-signaling differ greatly; while classical signaling is required for antimicrobial defenses, it appears that many of the pathological effects of IL-6-driven inflammation can be attributed to trans-signaling ${ }^{36}$. For example, it is mostly trans-signaling that drives gliosis, vasculopathy and neurodegeneration in IL-6-overexpressing mice ${ }^{37}$. Trans-signaling by IL-6 is also largely responsible for pathological immune cell infiltrate in murine arthritic models as well as osteoarthritis and rheumatoid arthritis patients ${ }^{38}$; indeed, sIL-6R expression is normally elevated in rheumatoid arthritis and cancers ${ }^{39}$.

Herein sgp130 presents an interesting layer to regulation. Notably, trans-signaling can be selectively blocked by sgp130, which acts as a sink for free sIL-6R but does not affect membrane- 
bound IL-6R. While free IL-6 expression is low in the steady state, sIL-6R occurs at about a 10fold excess while, in turn, sgp130 is often present in 5-10-fold excess. Since sgp130 also has a high affinity for the IL-6:sIL-6R complex with a $\mathrm{K}_{\mathrm{D}}$ of $1 \mathrm{pM}$, it is able to tightly bind the complex and thus tightly control trans-signaling. Since sgp130 interacts only with the IL-6:sIL-6R without any effect on classical signaling by mIL-6R, it is an excellent experimental tool to determine the dominant signaling pathway in IL-6-dependent disease phenotypes and is currently under clinical trial for selective blockade of IL-6-driven inflammation ${ }^{37,38}$. Several splice forms of sgp130 exist that are generated by alternative splicing or alternative polyadenylation that all bind IL-6:sIL-6R, albeit at different affinities and with different dimerization properties ${ }^{34,40,41}$.

\subsubsection{Splicing of IL-15 and its receptor controls stability and secretion}

IL-15 and its specific receptor chain, IL-15R $\alpha$, also exhibit interesting splicing patterns that modulate the strength of IL-15 signaling. IL-15 signals on many cells, though its main biological function is the homeostasis, function and activation of $\mathrm{CD}^{+}$lymphocytes and natural killer cells ${ }^{42}$. Two splice variants exist that encode either a long signal peptide or short signal peptide upstream of the same full-length IL-15 (LSP-IL-15 and SSP-IL-15) and have wildly different tissue expression patterns ${ }^{43-46}$ (Fig. 1.4). In addition, while the isoform with the short signal peptide is efficiently translated, it remains in the cytoplasm and nucleus; the isoform encoded with the long signal peptide is poorly translated, but it is transported through the Golgi apparatus, undergoes post-translational modifications and is efficiently secreted. Furthermore, SSP-IL-15 competes with LSP-IL-15 for binding to IL-15R $\alpha$ when all three are expressed in the same cell and destabilizes the IL-15:IL-15R $\alpha$ complex ${ }^{47}$. These highlight a major role for splicing in the regulation of IL-15 translation and secretion. Alternative exon configurations of IL-15 isoforms in mice are generated in activated immune cells that change expression and activity, 
including an internally spliced exon 5 that improves translational efficiency, a variant lacking exon 6 that competitively inhibits IL-15 receptor binding, and an isoform lacking $5^{\prime}$ end of exon 7 that ameliorates dermal infiltrates of immune cells and acanthosis normally induced by full-length IL$15^{48-50}$.

The cytokine IL-15 is normally not secreted in a monomeric form but rather held tightly by its unique receptor IL-15R $\alpha$, which is expressed on many cell types ${ }^{51}$. This complex further requires binding to the IL-2/15 $\beta \mathrm{R}$ and $\gamma \mathrm{C}$ subunits (hereafter referred to as the $\beta / \gamma \mathrm{C}$ complex) as to signal (Fig. 1.5). Early intracellular association mutually increases stability of both variants of IL-15 as well as IL-15R $\alpha$, allowing their secretion as a complex ${ }^{47,52}$. Interestingly, membranebound complexes of IL-15 and IL-15R $\alpha$ on monocytes are recycled in endosomes and able to transactivate neighboring $\mathrm{T}$ cells expressing only the complementary the $\beta / \gamma \mathrm{C}$ complex ${ }^{53}$. IL- $15 \mathrm{R} \alpha$ undergoes diverse splicing in human monocytes and dendritic cells to yield 8 different isoforms with several combinations of an exon 2 insertion and alternative C-termini that alter glycosylation, maturation, secretion and trans-presentation of the receptor ${ }^{54}$. Additional isoforms generate alternative start sites and exclude exon 2, disrupting the sushi domain which is essential for posttranslational modification and cytokine binding ${ }^{55}$ (Fig. 1.5A).

Like IL-6R, IL-15R $\alpha$ is expressed in both transmembrane and soluble forms, and shedding of the soluble form from IL-15R $\alpha$-expressing cells can occur in proteolysis-dependent and independent mechanisms ${ }^{56}$. The heterodimeric complex of soluble IL-15 and soluble IL-15Ra comprises the majority of bioactive IL-15 in serum, as the soluble IL-15 is comparatively more unstable and less agonistic in its monomeric form ${ }^{57,58}$ (Fig. 1.5B). Indeed, addition of soluble IL$15 \mathrm{R} \alpha$ is able to enhance IL-15 agonism of lymphocyte proliferation, unlike the closely related soluble IL-2Ra which antagonizes IL-2 signaling ${ }^{59}$. 


\subsubsection{Splicing of IFN genes and their receptors}

Interferons are a family of cytokines that have essential, particular antiviral activities. They are largely categorized as type I (IFN $\alpha 1-14, \beta, \varepsilon, \kappa, \omega)$, type II (IFN $\gamma$ ) and type III (IFN $\lambda$ ). As one of the genes in interest of this thesis is the most recently discovered of these classes, IFN $\lambda 4$, a brief discussion of known splice variation in the cytokine and receptor genes follows to provide context for the unique splicing properties of IFN $\lambda 4$.

Compared to other cytokine families, splicing and alternative isoform generation of IFN genes is highly limited. Generally, splicing diversity and the biological effects of splice variation has not been explored in IFNs, although it has been sporadically examined in their receptors. Importantly, the question is moot in type I IFNs because nearly all transcripts arise from a single exon; thus, variation by splice variation is impossible. The contrasting multi-exon configuration of type III IFNs may hint at functional differences between the families despite highly similar downstream effector pathways. Of these, IFN $\lambda 1$ and IFN $\lambda 2$ only have one transcript annotated, while IFN $\lambda 3$ has two transcripts, although their functional differences have not been discovered. IFN $\gamma$, the sole representative of type II IFNs, is a multi-exon gene for which only one transcript exists. Therefore, IFN $\lambda 4$ is highly unique among all the IFN families for having several endogenously expressed splice variants. These are further discussed in Section 3.1.

The IFN family receptors demonstrate more splicing diversity than their ligands, although the true extent of variation and consequences are also poorly understood. In the case of type I IFN receptors, no natural variant of IFNAR1 has been identified; in contrast, multiple isoforms of IFNAR2 have been described that are generated by alternative splicing and polyadenylation. A soluble receptor lacking the transmembrane domain has been characterized in both humans and mice, and the latter has both agonistic and antagonistic roles in IFN signaling in vivo ${ }^{60-64}$. The type 
III IFN receptor, IFNLR1, also has two additional known isoforms: one isoform excludes exon 6 and generates a frame-shifted, truncated soluble receptor that inhibits type III IFN-dependent signaling, while a second isoform uses an alternative splice site to delete part of of exon 7. Both bind IFN $\lambda$ but do not signal ${ }^{65,66}$. Given that these isoform transcripts have been detected in human hepatocytes, keratinocytes, airway epithelium and immune cells, with the soluble receptor expressed at different proportions in different tissue, it is tempting to speculate that the choice of splice variant for IFNLR1 impacts these cells' responsiveness to IFN $\lambda$ stimulation and, therefore, their propensity for antiviral effector functions. The two receptor chains for type II IFNs, namely IFN $\gamma \mathrm{R} 1$ and IFN $\gamma \mathrm{R} 2$, have multiple annotated splice forms that have not been explored in detail. Perhaps the increased splice variation of IFN receptors in general compensates for the lack of ligand isoforms to preserve alternative splicing as a mechanism for controlling IFN signaling.

\subsection{SPLICING AS AN IMPORTANT PARADIGM OF IMMUNE REGULATION}

Lessons from IL-6 and IL-15 reveal a large variety of ways in which splicing can change immune function. These are particularly complex examples, although it is clear from other immune genes and cytokines that splicing causes the removal of important functional domains and generates competitive inhibitors for signaling ${ }^{27,29,67}$. Furthermore, as discussed in section 1.1.3, we still lack a "splicing code" for computational prediction of the strength of each splicing site, much less combinations of exons used under specific environments. Therefore, the expression of specific isoforms in a given context still has to be determined empirically for each specific gene under experimental conditions. While some genes, such as $C F T R$, have been extensively studied thanks to comprehensive annotation of databases,

In my thesis, we have chosen to examine two molecules for which alternative splicing is likely to have important functional effects but remains poorly studied. IL-22 binding protein (IL- 
22BP; gene symbol IL22RA2) is a soluble receptor for the IL-10 family cytokine IL-22 that has two special properties: first, it is the only soluble receptor known in the IL-10 family of cytokines, and second, it is encoded on a separate gene from the membrane-bound receptor for IL-22. While IL-6R, gp130 and IL-15R $\alpha$ have provided excellent examples for complex roles of soluble receptors, they are generated by alternative splicing (or in the case of the IL-6 receptor, limited proteolysis) from the same gene as the membrane-bound receptors. While genetic associations have implicated a role for $I L 22 R A 2$ in autoimmune disease, these do not distinguish between the three known splice variants of the receptor. It is likely that the alternative splicing of IL-22BP changes its function, localization or secretion, and these would be important to determine in the context of other alternatively spliced cytokine receptors.

Our second gene of interest, IFNL4, is a novel member of the IFN pathway that uniquely has multiple naturally-expressed transcripts; apart from the full-length protein, the functions of the isoforms are not known. The endogenous expression of isoforms contrasts other type III IFN genes that do not appear to undergo functional splicing. Since genetic associations already implicate a non-canonical function for IFN $\lambda 4$, it is likely that alternative splicing is an important mechanism that controls its gene expression as well as the canonical or non-canonical functions of IFN $\lambda 4$ that may be affected by exon inclusion or exclusion.

In our explorations of IL-22BP and IFN $\lambda 4$ in the following chapters, we will characterize both the functions of the alternatively spliced isoforms and their expression, in order to determine when these isoforms are expressed and what their effects are on the overall signaling pathways of these genes. In doing so, we add new complexity to the regulation of IL-22 signaling and the control of IFN activity. We expect that these mechanisms, which have parallels to known 
phenomena in other genes yet are relatively novel in the field of cytokines and interferons, will lay foundations for new perspectives on the control of immune signaling. 


\section{$1.4 \quad$ FIGURES}

A
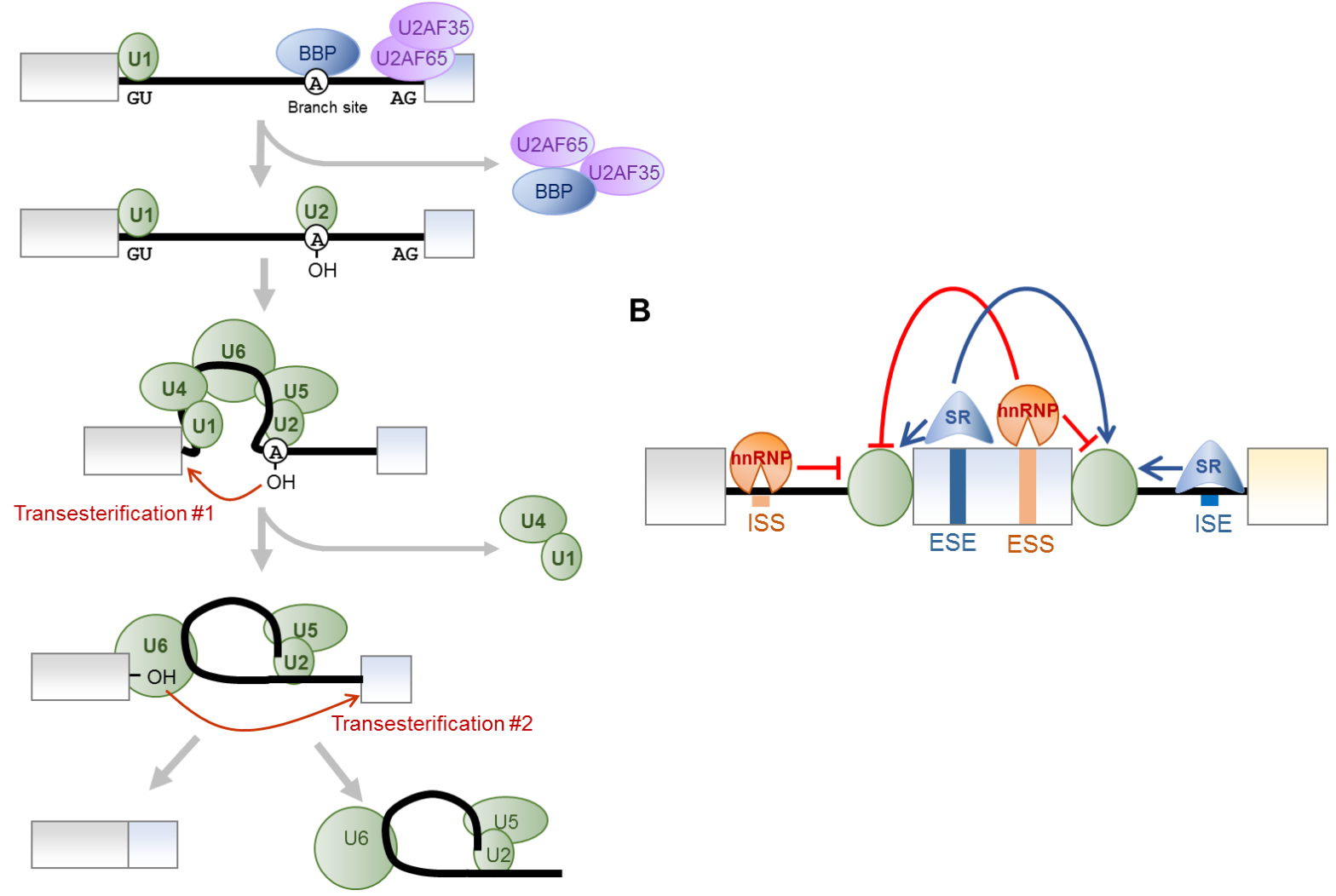

Figure 1.1. The processes of constitutive and alternative splicing.

(A) General mechanism of constitutive splicing. The core spliceosomal complex comprises the $\mathrm{U} 1, \mathrm{U} 2, \mathrm{U} 4, \mathrm{U} 5$ and U6 snRNPs and associated proteins that identify several important sequences: the $5^{\prime}$ splice site (GU), the $3^{\prime}$ splice site (AG) and an intronic branch site proximal to the 3' splice site. Upon recognition of the splice sites and branch site, U2 is recruited to the branch site where it and hydrolyzes the branch point adenosine residue. This initiates a cascade that recruits the U4-U5-U6 tri-snRNP complex and catalyzes the first transesterification reaction to yield the intron lariat. The U2, U5 and U6 snRNPs then guide the second transesterification process that ligates the two exons and releases the intron lariat.

(B) Mechanism of decision-making during alternative splicing. Not all splice sites are fully used. Choice of "weaker" splice sites depends on the association of trans-acting factors, such as hnRNPs and SR proteins, with cis-regulatory elements: intronic and exonic splice enhancers (ISE/ESE) and suppressors (ISS/ESS). The positive and negative signals are processed by the spliceosome to define whether the exon is included or excluded. 
Alternative cassette exon (exon inclusion/skipping)

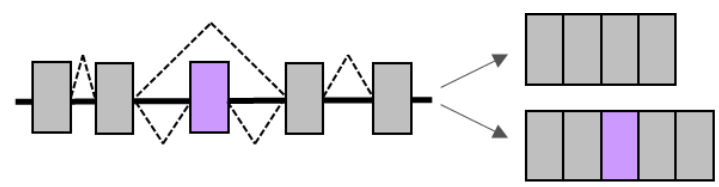

Mutually exclusive exons (exon inclusion/skipping)
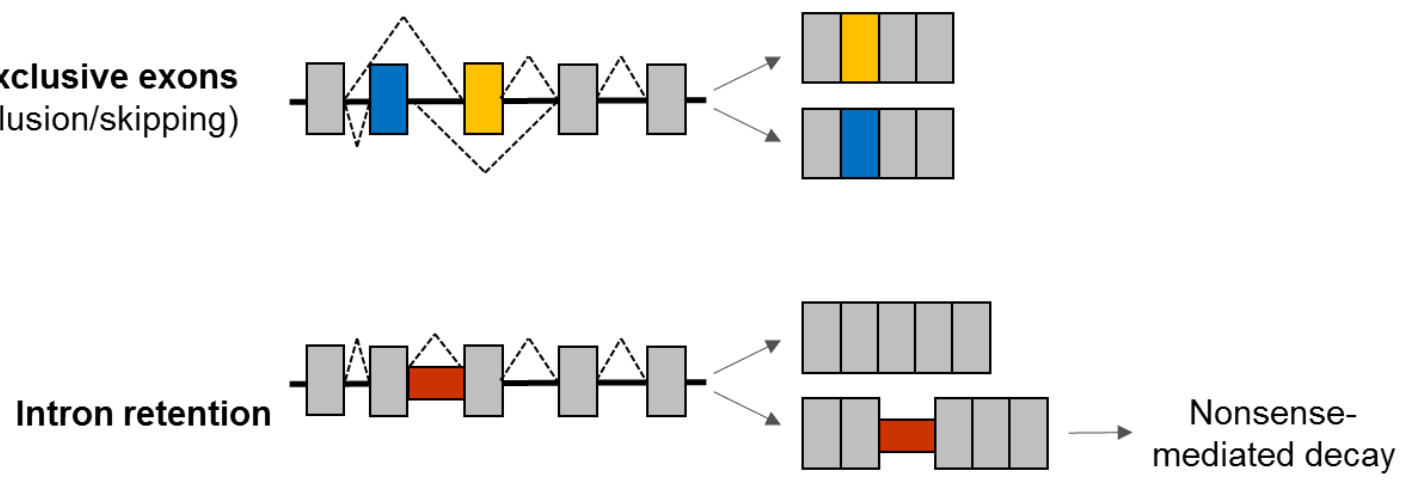

Alternative 5' splice site

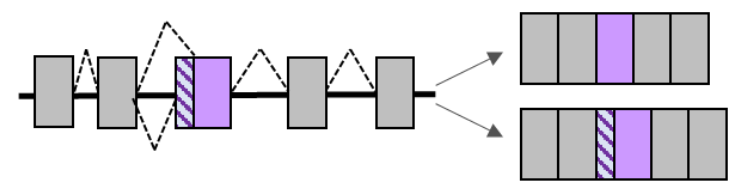

Alternative 3' splice site

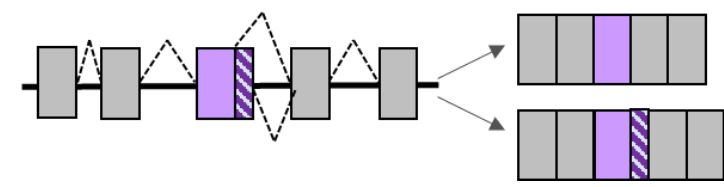

Figure 1.2. Outcomes of alternative splicing.

Schematics showing multiple ways in which alternative splicing can occur, and their effects on the resulting transcript. Alternative exon usage allows exon inclusion or exclusion; mutually exclusive exons generate different combinations; intron retention targets the transcript for nonsense-mediated decay; and alternative $5^{\prime}$ or $3^{\prime}$ splice site usage generates insertions or deletions that may keep the transcript in-frame or create a frame-shift that sometimes truncates the protein. Multiple mechanisms can be used the same gene individually or in combination. Grey boxes represent constitutive exons; purple, blue, gold and shaded boxes represent alternative exons; shorter red boxes represent retained introns. 


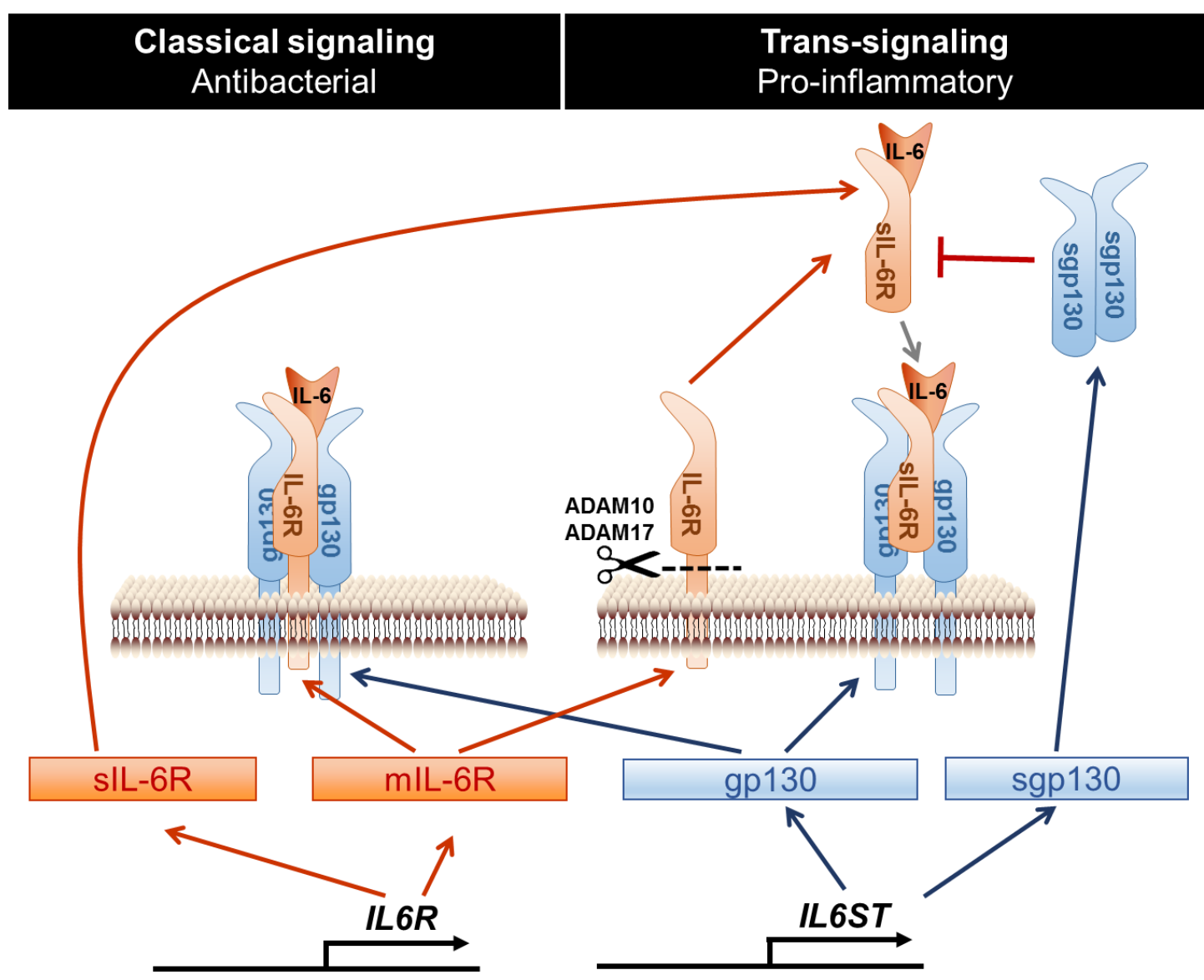

Figure 1.3. Alternative splicing of the IL-6 receptor increases diversity in IL-6 signaling.

Schematic of IL-6 membrane-bound receptors, soluble receptors and how they regulate one another to cause either classical or trans-signaling. Both IL-6R and gp130 (gene symbol IL6ST) have membrane-bound and soluble isoforms that can be generated by alternative splicing or, in the case of IL-6R, proteolysis by ADAM10 or ADAM17. Classical signaling through both membrane-bound receptors is limited to hepatocytes... that express full-length IL-6R (mIL-6R) expression. In contrast, trans-signaling occurs when soluble IL-6R (sIL-6R) binds IL-6 and then interacts with gp130 on cells lacking mIL-6R. Due to the ubiquitous expression of gp130, transsignaling can occur on many cell types. Importantly, each mode of signaling has divergent effector functions: while classical signaling is required for the beneficial antimicrobial activites of IL-6, pathogenic pro-inflammatory signaling associated with autoimmunity and neoplasia seems to be solely dependent on trans-signaling. Soluble gp130 (sgp130) specifically inhibits IL6 trans-signaling but leaves classical IL-6 signaling intact; its high concentration provides a physiological buffer to limit IL-6 trans-signaling in the steady state. 


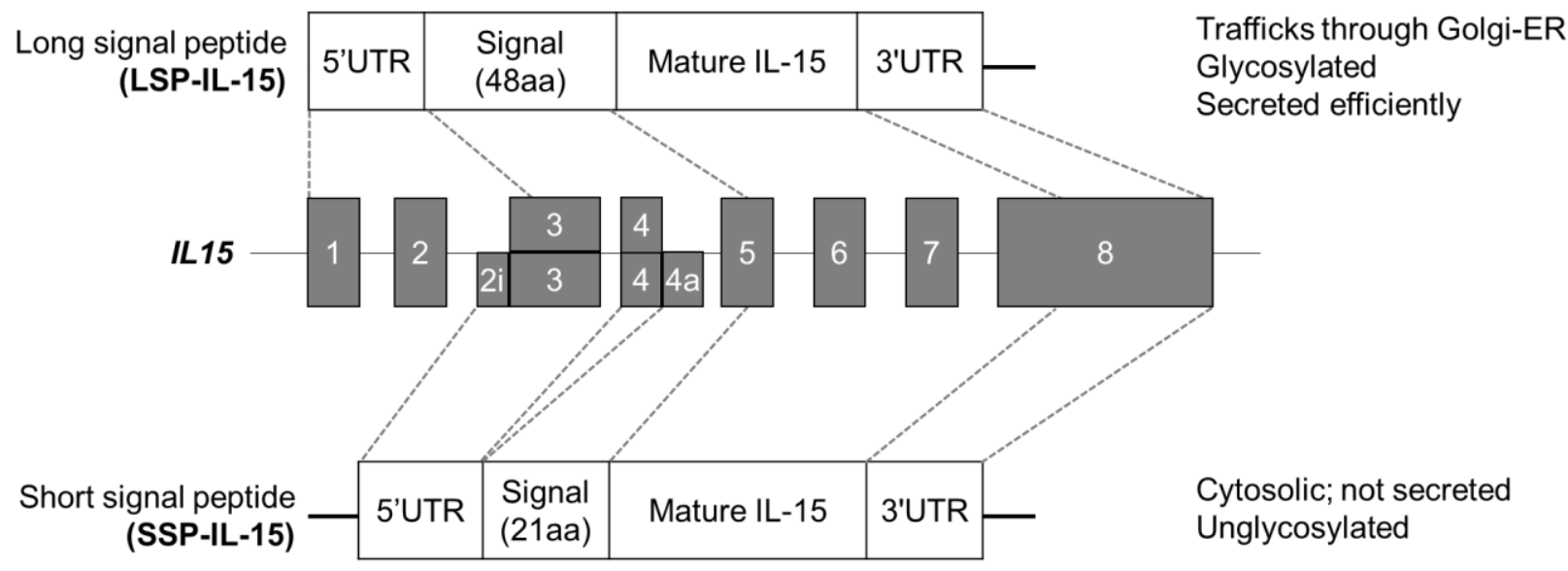

Figure 1.4. Alternative splicing of IL-15 changes its localization and post-translational modification.

Schematic of the IL15 transcript and generation of two major isoforms by alternative splicing. Use of different transcription start sites generates two transcripts of IL-15 encoding the same mature peptide with two different lengths of signal peptide (long and short, for LSP-IL-15 and SSP-IL-15 respectively). The long signal peptide is able to target the protein for transport through the endoplasmic reticulum and Golgi apparatus, but not the short peptide. As a result, LSP-IL-15 is glycosylated and efficiently secreted, while SSP-IL-15 remains unglycosylated and intracellular. Relative expression of LSP-IL-15 and SSP-IL-15 regulates the export of bioactive IL-15. In addition, SSP-IL-15 competes with LSP-IL-15 for binding to IL-15R $\alpha$ intracellularly and destabilizes the receptor. Therefore, alternative splicing to generate SSP-IL-15 instead of LSP-IL-15 creates a natural antagonist of IL-15 signaling. 


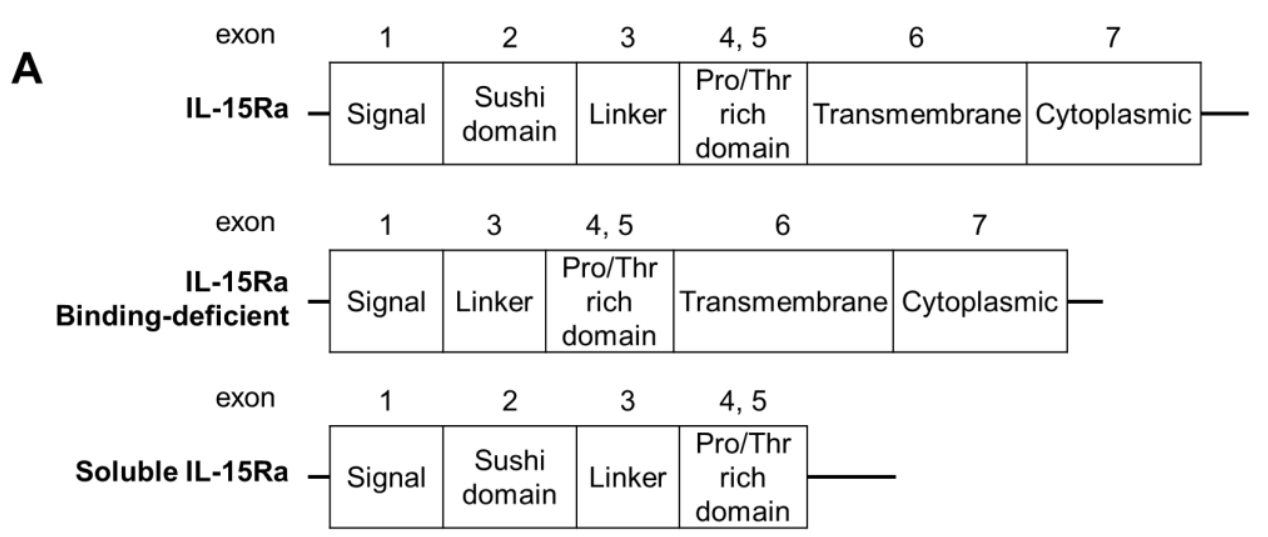

B

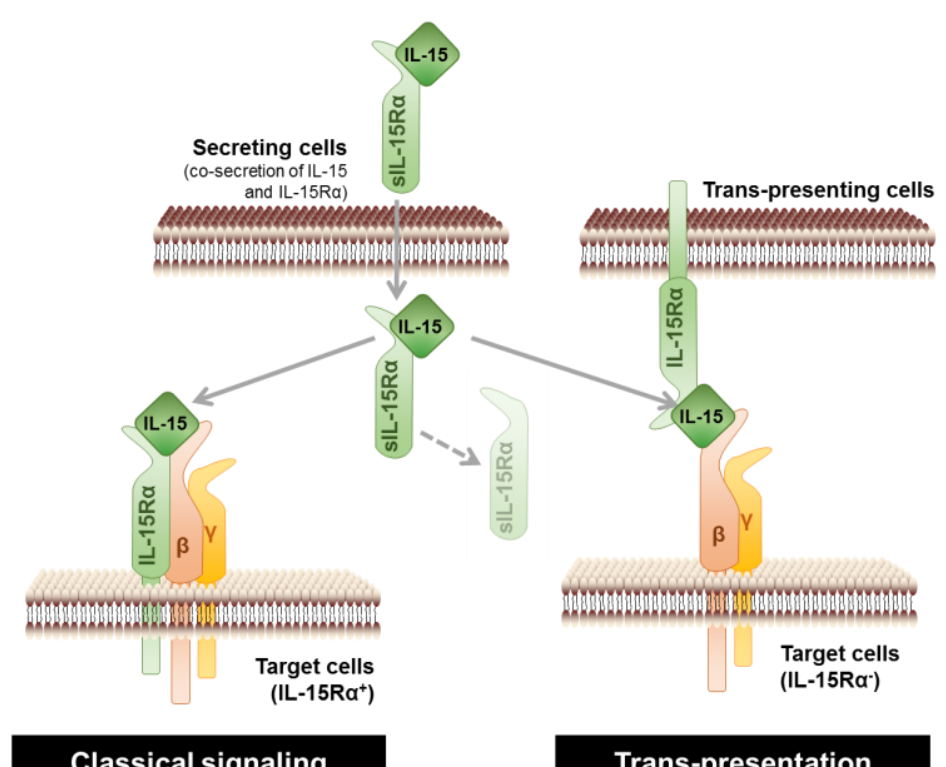

Figure 1.5. Alternative splicing of IL-15R $\alpha$ generates a soluble receptor that stabilizes IL15.

(A) Major domains encoded by exons of IL-15R $\alpha$ and splice variants. Two major isoforms have been studied: one lacking the sushi domain that is essential for cytokine binding (exon 2), and another lacking the transmembrane and intracellular domain (exons 6 and 7). (B) Schematic of signaling modes through IL-15/IL-15R $\alpha$, and the role of soluble IL-15R $\alpha$. This complex associates intracellularly to increase stability of both proteins and remains bound in serum. IL-15 binds membrane-bound IL-15R $\alpha$, IL-2/15R $\beta$ and common $y$ chain (the $\beta y$ complex) on the target cell to signal. In classical signaling, IL-15R $\alpha$ is expressed on the same cell as the $\beta \gamma$ complex. In trans-presentation. IL-15 is able to bind the $\beta \gamma$ complex on a target cell due to stabilization and presentation by an adjacent cell. Trans-presentation is an important mechanism for IL-15 signaling in lymphocytes, which require IL-15 activity for homeostasis, activation and memory formation despite a lack of cell-intrinsic IL-15R $\alpha$ expression. 


\section{Chapter 2. THE HUMAN IL-22 BINDING PROTEIN ISOFORMS ARE A RHEOSTAT FOR IL-22 SIGNALING.}

Parts of the following work has been accepted for publication in Science Signaling as a scientific article by authors Chrissie Lim, MeeAe Hong and Ram Savan. Additionally, sections of the introduction have been published in Cytokines and Growth Factor Reviews as a review by authors Chrissie Lim and Ram Savan. Text and figures have been reproduced with permission. (C) American Association for the Advancement of Science, 2016; Elsevier, 2013.

The cytokine interleukin-22 (IL-22) activates signal transducer and activator of transcription 3 (STAT3) to drive various processes central to tissue homeostasis and immunosurveillance; its dysregulation causes inflammatory diseases. IL-22 has a soluble receptor, IL-22 binding protein (IL-22BP; IL22RA2), which antagonizes IL-22 activity and has genetic associations with autoimmune diseases. Humans express three IL-22BP isoforms, whereas mice only express an IL22BPi2 homolog. Given the lack of IL-22BPi1 and IL-22BPi3 expression in mice, there has not been a thorough characterization of their activities to date. In this study, we show that IL-22BPi2 and IL-22BPi3 differentially modulate IL-22 activity: IL-22BPi3 has lower inhibitory activity than IL-22BPi2, but is expressed more abundantly throughout the body during homeostasis. IL-22BPi2 and IL-22BPi3 also differentially alter the cooperative actions between IL-22 and IL-17, which are commonly co-expressed in autoimmune settings. In addition, we demonstrate that IL-22BPi1 is inactive because it is not secreted. Importantly, while IL-22BPi3 is the dominant isoform expressed constitutively, only IL-22BPi2 is modulated when myeloid cells are activated by TLR2 or retinoic acid. These data suggest that the IL-22BP isoforms have distinct spatial and temporal roles and coordinately fine-tune IL-22-dependent STAT3 responses in tissues as a rheostat. This work is the first to date to perform a thorough biochemical and molecular characterization of the human IL-22BP isoforms. 


\subsection{INTRODUCTION}

IL-22 is an $\alpha$-helical class II cytokine of the IL-10 family cytokine secreted mainly by CD4 ${ }^{+}$ helper T cells, $\gamma \delta \mathrm{T}$ cells, innate lymphoid cells and NKT cells ${ }^{68-70}$. IL-22 signaling through its unique receptor IL-22R1 and the common IL-10 family receptor chain IL-10R2 activates principally STAT3 to induce transcription of genes with context-specific pro- or antiinflammatory, pro-survival, regenerative and anti-fibrotic effects ${ }^{71-78}$. IL-22 also activates STAT1 in specific contexts, particularly in the presence of interferons, to mediate antiviral activity and inflammation in the gut epithelium ${ }^{74,79-81}$. Therefore, IL-22 plays important roles in tissue repair, mucosal immunity and metabolic activities ${ }^{75,78,82-93}$. Dysregulated expression of IL-22 drives inflammation in psoriasis and Crohn's disease $\mathrm{e}^{72,74,76}$. IL-22 also has complex, stage-specific proand anti-tumor effects ${ }^{94}$. Consequently, IL-22 inhibitors are of significant clinical interest and currently under trial in the treatments of psoriasis, rheumatoid arthritis and atopic dermatitis (www.clinicaltrials.gov).

IL-22 also has a soluble receptor, IL-22 binding protein (IL-22BP; gene symbol IL22RA2). Studies in $I l 22 \mathrm{ra}^{-\digamma}$ mice have revealed striking effects in colitis and experimental autoimmune encephalitis $(\mathrm{EAE})^{78,95-97}$, pointing to a crucial role for IL-22BP in the control of pathological IL22 signaling. However, data from mouse models have not translated into understanding the mechanisms behind the associations with human disease. This is likely because IL22RA2 encodes three alternatively-spliced isoforms in humans named as IL22RA2v1 (IL-22BPi1), IL22RA2v2 (IL22BPi2) and IL22RA2v3 (IL-22BPi3) but only one isoform in mice, Il22ra2 (Il-22bp), which is homologous to human IL22RA2v2/IL-22BPi2 (Fig. 2.1; Suppl. Fig. 2.1). Knowledge about IL22BP is largely limited to the shared murine and human homolog, IL-22BPi2, which inhibits IL- 
22 signaling in vitro ${ }^{98,99}$. However, the control of IL-22BP expression remains poorly understood. Furthermore, the biological functions of IL-22BPi1 and IL-22BPi3 remain uncharacterized.

In this chapter, we use molecular and biochemical approaches to define the functions of IL-22BPi1 and IL-22BPi3 in comparison to IL-22BPi2, and go on to profile the distinct expression of the isoforms in homeostasis and inflammation. We expect that our findings will add invaluable insight to how these human-specific isoforms contribute to disease progression in patients and begin to provide possible functional explanations for genetic associations for disease.

\subsubsection{IL-22 performs pleiotropic, context-dependent functions}

The IL-22 gene (gene symbol IL22) is located on chromosome 12 in humans, in close proximity to the loci encoding interferon-gamma (IFN- $\gamma$; gene symbol IFNG) and IL-26 ${ }^{100}$. IL-22 has also been cloned from non-mammals such as fish, where its chromosomal location between the IFNG and IL26 loci remains conserved ${ }^{101}$. Thus we can infer that its importance to homeostasis and immunity has persisted for a long time in evolutionary history.

Principal sources of IL-22 include $\mathrm{CD}^{+}$helper T cells, CD8 ${ }^{+}$cytotoxic T cells, $\gamma \delta \mathrm{T}$ cells, innate lymphoid cells (ILCs) encompassing natural killer (NK) cells and lymphoid tissue inducer (LTi)-like cells ${ }^{68,69}$, and NKT cells which express both NK cell markers and T cell receptor ${ }^{70}$. It is important to bear in mind that the principal cellular sources of IL-22 varies widely between tissues, so regulation of IL-22 production and signaling may involve targeting different immune cell populations or niches. For example, the expression of CCR10 on Th22 cells but not Th17 cells causes preferential recruitment of Th22 cells to the skin where they are the primary source of IL$22^{102-104}$, whereas they are overshadowed by $\gamma \delta \mathrm{T}$ cells and ILCs in the gut ${ }^{105-107}$. In immune challenge, kinetics also dictate the main cellular sources of IL-22: during early response, IL-22 production primarily comes from ILCs and tissue-resident $\gamma \delta \mathrm{T}$ cells which are poised for rapid 
activation, while later on the adaptive immune system provides a second wave of IL-22 from conventional $\alpha \beta T$ cells ${ }^{108,109}$. This is likely why different IL-22-producing subsets are major players for different models of colitis, particularly with respect to the innate/adaptive immune divide $^{110}$. The local milieu and disease kinetics will be major factors to account for when dissecting IL-22-dependent mechanisms of disease. A summary of principal sources of IL-22 and its downstream signaling effects is presented in Fig. 2.2.

Although IL-22 is secreted exclusively by immune cells, it signals solely on non-immune cells including hepatocytes, keratinocytes, lung epithelial cells, colonic epithelial cells and pancreatic acinar cells, to which IL-22R1 expression is restricted ${ }^{71,75-77,85,111-117}$. IL-22R1 is also expressed in some other tissue cells such as hepatic stellate cells ${ }^{118}$, colonic subepithelial myofibroblasts ${ }^{119}$, smooth muscle cells ${ }^{120}$ and thymic epithelial cells ${ }^{121}$, but their sensitivity to IL22 stimulation appears to vary. IL-22 signals through a heterodimeric receptor comprising its unique receptor IL-22R1 (gene symbol IL22RA1) and IL-10R2 $2^{114,122}$ to activate Signal Transducers and Activators of Transcription (STATs) 1, 3 and 5, nuclear factor kappa B (NF-кB), mitogenactivated protein kinase (MAPK) and phosphatidylinositide 3-kinase-Akt-mammalian target of rapamycin (PI3K-Akt-mTOR) pathways ${ }^{109,123}$. The consequences of these signaling pathways are detailed below. Their variety demonstrate the profound effects of IL-22 signaling throughout the body as well as the great need to learn how it can be controlled in specific tissues and situations.

\subsubsection{Signaling effects downstream of IL-22/IL-22R1}

IL-22 stimulation induces expression of a medley of genes principally downstream of STAT3, with pro-inflammatory, anti-inflammatory, mitogenic, pro-survival, anti-apoptotic or anti-fibrotic effects depending on specific tissue and disease context $\mathrm{t}^{108,109,124}$. Because immune cells are not responsive to IL-22, it has generated immense interest as a therapeutic target with 
likely fewer side effects than IL-6, IL-17, IL-21 or other principal STAT3-activating cytokines ${ }^{109}$. However, its pleiotropic functions throughout the body still make it a challenging cytokine to manipulate for treatment of disease. For example, the IL-22/IL-22R1 has complex effects on carcinogenesis and tumor progression that are origin- and stage-specific; while anti-inflammatory effects of IL-22 can reduce initial tumorigenesis, prolonged dysregulation and activation can act as a proliferative driver of established cancers ${ }^{94}$. Furthermore, IL-22 can either be protective or pathogenic in different murine colitis models ${ }^{110}$. Therefore, care must be taken in interpreting the contribution of IL-22 to disease progression.

The principal downstream effects of IL-22 are mediated via JAK/STAT signaling, mainly STAT3, which IL-22 activates through phosphorylation at both Tyr-705 and Ser-727 ${ }^{125}$. Constitutive association of STAT3 molecules with the intracellular domain of IL-22R1 poises the cell for rapid activation, though activation also requires association of Src Homology Phosphatase 2 (SHP2) with activated IL-22R1 at phosphorylated residues Tyr-251 and Tyr-301 ${ }^{126,127}$. IL-22 signaling enacts negative feedback through induction of suppressors of cytokine signaling 1 and 3 (SOCS1/3), which inhibiting STAT3 activity $^{123}$. On the other hand, since STAT3 enhances IL-22 production by Th17, enrichment of IL-6, IL-21, IL-23 and IL-1 $\beta$ in a STAT3-driven niche sustains local differentiation and maintenance of IL-22 signaling $^{102,128,129}$.

To a smaller extent, STAT1 activation by IL-22 is observed in some cells including hepatocytes, colonic epithelial cells and renal carcinoma cells ${ }^{74,75,81,114,115,125,130-132}$, and it can be enhanced by IFN $\alpha$ costimulation ${ }^{81}$. Rare STAT5 activation has also been observed in hepatocytes and kidney cells, but downstream effects, if any, are yet unknown ${ }^{113,114,123,125}$. Multiple MAPK pathways are activated downstream of IL-22R1 including the p38, extracellular signal-related kinase (ERK) and c-Jun N-terminal kinase (JNK) cascades, though sensitivity to induction, exact 
pathways activated and subsequent downstream events are varied and cell-type specific $^{119,120,125,127,130,131,133-136}$. In addition, IL-22 activates the PI3K-Akt-mTOR pathway in some cells. Akt activation via IL-22 is necessary for proliferation in normal human epithelial keratinocytes and fibroblast-like synoviocytes, while in renal tubular epithelial cells it inhibits mitochondrial apoptosis ${ }^{133,137}$. PI3K activation is also required for migration of colonic epithelial cells and hepatocytes ${ }^{74,123}$.

Lastly, IL-22 is able to functionally activate NF- $\kappa B^{119,120,138,139}$. Notably, NF- $\kappa \mathrm{B}$ can promote expression of genes involved in cell cycle, proliferation and prevention of apoptosis, particularly in synergy with STAT3 $3^{138,140,141}$. So far, inflammatory cytokine induction and proliferation have been observed downstream of IL-22 signaling via NF-kB ${ }^{119,120,138,139}$; the intersection of these pathways with STAT3 signaling may yet prove interesting especially for the role of IL-22 in transformation and cancer progression.

\subsubsection{Physiological roles of IL-22 signaling}

Since many IL-22-responsive cell populations are epithelia linings of mucosal barriers, it is no surprise that IL-22 has many pro-inflammatory effects to rapidly defend against invading pathogens. In hepatocytes and colonic epithelial cells, IL-22 potently induces an acute phase response including the induction of serum amyloid protein, $\alpha$-chymotrypsin and haptoglobin ${ }^{77,100}$. IL-22 also induces the production of $\mathrm{ELR}^{+}$chemokines CXCL1, CXCL2 and CXCL5 which recruit neutrophils to the site of invasion ${ }^{85,107,118,142,143}$. IL-22 instigates the production of inflammatory cytokines such as IL-6, IL-8, TNF $\alpha$ in various cells including hepatic stellate cells, myofibroblasts and keratinocytes; enhanced processing of the NLRP3 inflammasome also yields IL-1 $\beta^{118,119,144}$. This further hints at oxidative signaling induced by IL-22, which employs NADPH oxidase to activate STAT3 and may synergize with IFN- $\gamma$ to produce nitric oxide species ${ }^{132,145}$. 
Furthermore, IL-22 stimulates the production of an impressive repertoire of antimicrobial peptides, including several members of the S100A family, the regenerating protein (RegI, RegII, RegIII) families, lipocalin, defensins and the cathelicidin LL-37 in the skin and colon ${ }^{71-73,84,85,146,147}$. IL-22 suppresses differentiation-related tissue factors including loricrin, involucrin and calmodulin in keratinocytes, which together with its inflammatory activites drives progression of psoriasis $72,76,83,143,148,149$. In addition, IL-22 stimulates mucin production in colonic epithelium cells 78. While these actions are transiently pro-inflammatory, clearance of pathogens decreases the duration and extent of inflammatory signals ${ }^{109}$. IL-22 also suppresses IFN- $\gamma$-dependent induction of pro-inflammatory chemokines in vitro ${ }^{150}$.

In particular, IL-22 has specialized roles the maintenance of barrier immunity in the gut. IL-22 produced by ILCs in the lamina propria enhance lymphotoxin-alpha production as one method to increase barrier protection; commensal microbiota enhance IL-22 activity to increase fucosoylation that prevents colonization by invasive Salmonella typhimurium and Enterrococcus faecalis, preventing systemic septicaemia caused by spread of the latter ${ }^{89,151}$. Serum amyloid protein induction by IL-22 from innate lymphoid cells is also essential for the maintenance of Th17 barrier immunity ${ }^{152}$.

Metabolic functions of IL-22 have been more recently characterized. IL-22 induces hepcidin in hepatocytes, an enzyme which decreases iron bioavailability in blood ${ }^{153,154}$. IL-22 also decreases expression of lipogenetic transcription factors and genes in the liver in a hepatic steatosis $\operatorname{model}^{155}$.

While IL-22 itself has pleiotropic pro- and anti-inflammatory roles in different sites and environments, interactions and cooperation with other cytokines in the environment can alter its function. The balance of pathogenic and protective functions of IL-22 in a bleomycin-induced lung 
inflammation model depended on the presence of IL-17 in the microenvironment: while IL-22 enhanced inflammation in the presence of IL-17, IL-22 was required for tissue protection in the absence of IL-1 $17^{156}$. Thus, IL-22 is able to exert both pro- and anti-inflammatory actions in synergy with the local cytokine milieu. IL-22 is also able to synergize with type III IFNs to enhance antiviral immunity against rotaviral infection ${ }^{80}$. Interactions between IL-22 and other cytokines are a major factor in determining the outcomes of its signaling.

\subsubsection{The soluble receptor IL-22 binding protein (IL-22BP)}

IL-22 is unique among the IL-10 family of cytokines to have a soluble receptor, IL-22 binding protein (IL-22BP; gene symbol IL22RA2) ${ }^{98,157-160}$. IL-22BP is highly expressed in the placenta and mammary gland; found at intermediate levels in the stomach, lung, skin, spleen, thymus and lymph nodes; and modestly expressed in peripheral blood leucocytes, prostate, heart and brain tissues ${ }^{98,157-160}$. Only recently have myeloid dendritic cells been confirmed as a specific source, from which IL-22BP is induced by all-trans retinoic acid and regulated by IL-18 through the NLRP3/NLRC4 inflammasomes ${ }^{96,161}$. While genetic associations between IL22RA2 and autoimmune disease, inflammatory disease or cancer have been identified ${ }^{131,162-166}$, the role of IL22BP in vivo remains an enigma. IL-22 and IL-22BP expression is inversely regulated in dextran sodium sulfate (DSS) colitis models ${ }^{78,96}$. IL-22BP protein levels were elevated in the cerebrospinal fluid of patients with active multiple sclerosis, while there was a trend of increased transcript expression in their monocytes alongside increased IL-22 levels in their PBMCs ${ }^{167}$. Patients with active lupus nephritis in a small study also had increased levels of IL-22BP in urine to accompany increased IL-22 in serum, although IL-22BP levels in serum did not differ from controls ${ }^{168}$. Given that IL-22BP was protective in a colitis-associated colon carcinoma model yet detrimental to the progression of $\mathrm{EAE}^{96,97}$ despite a pathological role for IL-22 in both, it is likely an active member 
of the IL-22/IL-22R1 axis in health and disease and its role is likely dependent on tissue and inflammatory context, as is that of IL-22.

Notably, IL-22BP exists as three splice variants in humans (Fig. 2.1; Suppl. Fig. 2.1). Human isoforms of IL-22BP have demonstrated different binding affinities to IL-22 ${ }^{169}$, which will be referred to from here as IL-22BP isoforms 1, 2 and 3 (IL-22BPi1, 2 and 3). Murine IL22BP is homologous to IL-22BPi2, which is the isoform that has been studied almost exclusively. Compared to IL-22BPi2, IL-22BPi1 contains a unique exon 3 insertion while IL-22BPi3 lacks exon 5 and has a truncated exon 6 which comprises 5 frame-shifted amino acids with a premature stop codon. IL22RA2 sequences have been annotated for many vertebrates, but splice variation appears to be relatively recent. Indeed, exon 3 of IL-22BPi1 is encoded in a long terminal repeat retrotransposon introduced in rhesus macaques, which was later exonized in orang utans through the generation of a cryptic splice site by an $A>G \operatorname{transition~}^{170}$. This source explains why the isoform is absent in rodent models, which completely lack the genomic sequence containing exon $3^{159,170}$.

Studies to date have nearly exclusively studied IL-22BPi2, likely due to its homology with the murine protein for which the crystal structure has been solved. Despite poor amino acid sequence identity and location on a different chromosome from both IL22 and IL22RAI, it folds in a highly similar structure to the extracellular domain of IL-22R1, sharing crucial amino acid interactions for IL-22 binding but with much higher affinity and specificity ${ }^{99,169,171}$ (Fig. 2.1). IL22BPi2 has therefore been employed extensively in vitro as a competitive inhibitor of IL-22 signaling. The sole biochemical characterization of the other isoforms remains a surface plasmon resonance study which found that IL-22BPi3 binds to IL-22 with a similar affinity to the membrane bound receptor IL-22R1, both a thousand fold lower than that for IL-22BPi2 $2^{169}$. 


\subsubsection{Research objectives on IL-22BP and its isoforms}

Clearly, plenty of questions remain on the functional relevance of IL-22BP and its isoforms. Much of current knowledge about IL-22BP and IL22RA2 is restricted to genetic associations and biochemical assays, with little information of how it functions in biological systems. Even so, the small number of studies performed in vitro and in vivo study only exclusively IL-22BPi2; while its antagonism of IL-22 is consistent and reproducible in vitro, the divergent effects of IL-22BP on IL-22-driven murine colitis and EAE suggest a more complicated role in vivo.

Furthermore, the question of the isoforms in humans adds an important layer to the study of IL-22BP and how it interacts with IL-22. The roles of IL-22BP isoforms have largely been ignored due to the lack of isoform-specific reagents in humans and splice variants in the mouse; indeed, many present studies employ immunostaining or quantitative PCR approaches that do not distinguish between the isoforms. Unfortunately, neither genomic variants nor $I l 22 \mathrm{ra}^{-1}$ mice effects or dominance of these human-specific isoforms. Functionally, pharmacological binding affinity also does not determine biological relevance due to localization of the protein or more complicated receptor-ligand interactions that can cause trans-presentation, as in IL-6 where cisand trans-activation results in different gene transcription programs. Determining the separate functions of IL-22BP isoforms will be essential for mechanistic insights into the genetic associations linking IL22RA2 and human disease. Lastly, whether the expression of each isoform is distinctly expressed needs to be determined so that it is clear where the signaling effect of each isoform is dominant.

In this chapter, we identify distinct roles for the IL-22BP isoforms by investigating their molecular and biochemical activities. We demonstrate that IL-22BPi3 is able to functionally block 
IL-22 signaling, but is more dominant in weak homeostatic control of IL-22 while expression of the stronger antagonist IL-22BPi2 is modulated in inflammatory conditions. By combining function and expression to determine how, where and when the IL-22BP isoforms affect IL-22 signaling, we propose a model in which IL-22BPi2 and IL-22BPi3 collaborate to set the threshold for activation of IL-22 signaling and cooperation with other cytokines in human health and disease.

\subsection{Results}

\subsubsection{IL-22BP isoforms exhibit distinct activities against IL-22 signaling.}

The functions of IL-22BPi1 and IL-22BPi3 and their effects on IL-22 signaling remain unknown. As alternative splicing of IL22RA2 generates structural differences between IL-22BP isoform proteins (Fig. 2.1A, Suppl. Fig. 2.1), we compared their biochemical properties and functions. We over-expressed IL-22BPi1, IL-22BPi2 and IL-22BPi3 and observed expression of all the isoforms in cell lysates, but only IL-22BPi2 and IL-22BPi3 in the supernatants, even after concentrating supernatant proteins by trichloroacetic acid (TCA) (Fig. 2.1B). Treatment of lysates with PNGase F also revealed that all three isoforms are N-linked glycosylated, with double bands for IL-22BPi1 and IL-22BPi2 (Fig. 2.1C).

To assess how the IL-22BP isoforms affect IL-22 signaling, we tested the activity of IL-22 in the presence of IL-22BP isoforms. Briefly, we harvested the supernatants from cell cultures overexpressing IL-22BP isoforms and pre-incubated IL-22 with the supernatants to allow association. This mixture was transferred onto Huh7 hepatocytes. To measure downstream signaling, we performed immunoblots for the phosphorylation of STAT3, the main proximal signaling protein activated by IL-22 signaling ${ }^{72,74,75}$. We also measured expression of the IL-22inducible gene SOCS3 by real-time quantitative PCR (qPCR). IL-22BPi2 was able to block phosphorylation of STAT3 (Fig. 2.1D; Suppl. Fig. 2.2A) and IL-22-dependent SOCS3 induction 
(Fig. 2.1E) in Huh7 cells. Thus, overexpressed IL-22BPi2 effectively blocked IL-22 signaling in vitro, confirming prior studies ${ }^{98,99}$. IL-22BPi1 did not significantly alter IL-22 signaling (Fig. 2.1, D-E), which was expected given its absence in supernatants. More intriguingly, while IL-22BPi3 also antagonized IL-22 signaling, this blockade was weaker than that of IL-22BPi2.

\subsubsection{Inactive IL-22BPil is retained intracellularly due to its third exon.}

We were intrigued by the intracellular retention of IL-22BPi1 and its inactivity against IL22. We observed that the defect was at the stage of secretion as all the isoforms were detected in the cell lysates, but only IL-22BPi1 was absent from the supernatant (Fig. 2.1B). Since the coding sequences of IL-22BPi1 and IL-22BPi2 differ only by exon 3, we hypothesized that this sequence could be responsible for IL-22BPi1 retention within the cell. To test this, we sub-cloned exon 3 from IL-22BPi1 into IL-22BPi3-HA to generate an artificial 162 aa open reading frame, termed IL-22BPi3-ex3 (Fig. 2.1F). We then transfected this vector, along with those encoding IL-22BPi1 and IL-22BPi3, into cells to determine if this protein is secreted in the supernatant. We found that the insertion of exon 3 was sufficient to retain IL-22BPi3 intracellularly, even though it is normally secreted (Fig. 2.1G). We also found that insertion of exon 3 into IL-22BPi3 abolished the suppressive effect of IL-22BPi3 on IL-22 signaling as read out by a STAT3-dependent luciferase reporter (Fig. 2.1H; Suppl. Fig. 2.2B).

\subsubsection{Functional IL-22BP isoforms differentially modulate IL-22 activity.}

Overexpression systems do not allow us to control the molarity of IL-22BP isoform proteins provided in each experiment or to measure dose-dependent responses. To accurately compare the dose-dependent effects of human IL-22BP isoforms on IL-22 signaling, we purified recombinant human IL-22BPi3, using a eukaryotic Schneider 2 (S2) cell Drosophila expression 
system to retain glycosylation. We expressed IL-22BPi3 with a Histidine (6x) tag in the C-terminus under control of a copper (II) ion-inducible metallothionein promoter so that recombinant protein could be harvested and extracted from cell supernatant by affinity purification (Fig. 2.2, A-C). We then stimulated Caco-2 intestinal epithelial cells and Huh7 hepatocytes with IL-22 in the presence of recombinant human (rh)IL-22BPi3 in varying molar ratios to IL-22 as a dose curve of its effects on IL-22 activity. The blocking effect of rhIL-22BPi3 was compared against rhIL-22BPi2 applied in the same dose curve. We found that recombinant IL-22BPi3 is able to block IL-22 signaling in a dose-dependent fashion as assayed by phospho-STAT3 immunoblot in Huh7 and Caco-2 cells (Fig. 2.2D, Table 2.1). To precisely compare the antagonistic efficacy of the two isoforms, we measured the $\mathrm{IC}_{50}$ values of IL-22BPi2 and IL-22BPi3 acting on IL-22-dependent induction of STAT3-dependent luciferase reporter activity (Fig. 2.2E); for this constant dose of $10 \mathrm{ng} / \mathrm{ml}(0.606$ $\mathrm{nM}), \mathrm{IL}-22 \mathrm{BPi} 2$ had an $\mathrm{IC}_{50}$ value of $1.47 \mathrm{nM}$ (2.426-fold molar excess of IL-22, $\left.75.42 \mathrm{ng} / \mathrm{ml}\right)$, while IL-22BPi3 had an IC 50 of 40.22 nM (66.37-fold molar excess of IL-22, 58.32 ng/ml, $)$, giving an $\mathrm{IC}_{50}$ shift in 27.36 between the two isoforms. Similarly, IL-22BPi3 suppressed the induction of IL-22-dependent induction of SOCS3 in Huh7 cells (Fig. 2.2F) as well as CXCL5, DMBT1 and MUC1 (Fig. 2.2G) in Caco-2 cells. As expected from the $\mathrm{IC}_{50}$ values, IL-22BPi3 consistently showed weaker antagonistic efficacy than IL-22BPi2 supplied at the same molar ratio against IL22.

\subsubsection{IL-22BPi2 and IL-22BPi3 differentially skew the IL-17/IL-22 axis.}

Many IL-22-producing lymphocytes co-express IL-17, which has overlapping and cooperative functions with IL-22 but is a more potent inducer of pro-inflammatory genes ${ }^{73,143,172}$. Therefore, we assessed if the presence of IL-17 altered the effects of IL-22BP isoforms on IL-22 signaling. We incubated IL-17 and IL-22, individually and in combination, with either IL-22BPi2 
or IL-22BPi3 100-fold in excess of IL-22, then added the pre-incubated mixtures to Caco-2 cells to model co-stimulation of epithelial cells in physiological settings. We used DMBT1 as a readout for IL-22 activity, while $\beta 2$-defensin (gene symbols $D E F B 4 A / B$ ) indicated IL-17-specific activity. In turn, CXCL5 could be induced by either cytokine. While $\beta 2$-defensin induction has been shown in IL-22-stimulated keratinocytes ${ }^{71,73}$, we did not observe this induction in Caco-2 (Fig. 2.2H). $D M B T 1, D E F B 4 A / B$ and $C X C L 5$ were synergistically induced upon stimulations with IL-17 and IL-22. IL-22BPi2 and IL-22BPi3 acted specifically on IL-22 and did not affect IL-17 activity. IL22BPi2 was able to completely eliminate IL-22 activity while IL-17 signaling was retained. Incubation with IL-22BPi3 resulted again in a partial blockade, which dampened some of the synergy between IL-22 and IL-17 but did not abolish it (Fig. 2.2H).

\subsubsection{IL22RA2v3 is the widely expressed isoform during homeostasis.}

Given that IL-22BPi2 and IL-22BPi3 differentially modulate IL-22 activity, we surveyed the expression of all IL-22BP isoforms to determine where each signaling mode was dominant. To quantify the expression of IL22RA2 isoforms in humans, we designed qPCR probes that were specific for each isoform based on their unique exon usage (Fig. 2.3A). We obtained similar probe

efficiencies and specificity using expression vectors for each isoform and established standard curves for each probe to quantify copy numbers (Suppl. Fig. 2.3). We then performed quantitative PCR on a human tissue RNA panel to determine the expression of IL22RA2 isoforms (Fig. 2.3B). We found that IL22RA2v1 was expressed solely in the placenta. IL22RA2v2 expression was also highest in the placenta, but it was also detected at low levels in the spleen, stomach, thymus and thyroid gland. In contrast, IL22RA2v3 expression was widespread across many tissues and at higher copy numbers than the other isoforms even where co-expressed. 
To examine if there was any correlation between the expression of any IL22RA2 isoform and that of local tissue responsiveness to IL-22, we amplified the same tissue RNA for expression of the two membrane-bound receptor chains for IL-22, IL22RAI and IL10RB. Surprisingly, we found no correlation between the expression levels of any of the IL-22BP isoforms and the two membrane-bound receptor chains (Suppl. Fig. 2.4).

\subsubsection{IL22RA2v2 is the only isoform induced by the TLR2-retinoic acid pathway in myeloid cells.}

In addition to steady state expression, we sought to identify dominant IL-22BP functions in other immune environments. Myeloid cells were recently shown to be the main sources of IL22BP in the gut. Huber et al. (2012) showed that expression of Il22ra2 transcripts in the murine gut was correlated with that of CD11c, a commonly-used dendritic cell marker. Martin et al. (2014) then found IL-22BP was induced during in vitro differentiation of primary human monocytes into dendritic cells (DCs), and that expression was enhanced by retinoic acid treatment during differentiation $^{161}$. The same group also identified intestinal eosinophils as an additional source of human IL-22BP 95 .

Based on these reports, we used myeloid cells to determine if IL22RA2 isoforms were differentially regulated. We used undifferentiated THP-1 cells and THP-1 cells differentiated with GM-CSF and IL-4, which have been previously used as a model of DCs in vitro ${ }^{173,174}$. We confirmed induction of IL22RA2 in THP-1 cells upon retinoic acid stimulation, matching prior observations in primary monocyte-derived DCs ${ }^{161}$. Previously, IL22RA2v1 and IL22RA2v2 were observed in primary monocyte-derived DCs by reverse-transcriptase PCR ${ }^{161}$. While we detected IL22RA2v1 and IL22RA2v3 constitutively at low levels in all treatments, IL22RA2v2 was the predominant isoform expressed in myeloid cells (Fig. 2.3C). Furthermore, when treated with 
retinoic acid during differentiation, IL22RA2v2 was also strongly induced by stimulation, followed by marginal increases of IL22RA2v1, with minimal effect on IL22RA2v3 expression.

Retinal dehydrogenase 2 (RALDH2, gene symbol ALDH1A2) is an essential component to retinoic acid biosynthesis that is induced by stimulation of the pathogen sensor Toll-like receptor $2\left(\right.$ TLR2) ${ }^{175,176}$. Since retinoic acid enhances IL22RA2v2 expression, we hypothesized that TLR2 stimulation would induce IL22RA2v2 through RALDH2 activity (Fig. 2.3D). Indeed, stimulation of THP-1 cells with TLR2 agonist Pam3CSK4 induced the expression of IL22RA2v2 alongside $A L D H 1 A 2$, with no change in IL22RA2v1 or IL22RA2v3 (Fig. 2.3, E-F). THP-1-derived DCs, which basally express much higher levels of $A L D H 1 A 2$ than their undifferentiated counterparts, also further increased $A L D H 1 A 2$ and specifically IL22RA2v2 expression in response to Pam3CSK4 and lipopolysaccharide derived from E.coli 0111:B4 (LPS-EB) that activates both TLR2 and TLR4 (Fig. 2.3, G-H; Suppl. Fig. 2.5A). Since retinoic acid metabolism is important for multiple immune cell processes, particularly myeloid cell maturation ${ }^{177-179}$, we investigated whether IL22RA2v2 was also induced by TLR2 stimulation of other myeloid cell models. We differentiated HL-60 myeloid cells using dimethyl sulfoxide (DMSO) into a neutrophil model ${ }^{180-}$ 182 and stimulated them with Pam3CSK4 and LPS-EB. Again, while we detected all three isoforms before stimulation, only IL22RA2v2 was induced by treatment with Pam3CSK4 and LPS-EB (Suppl. Fig. 2.5, B-C). Importantly, stimulation of THP-1 monocytes and THP-1 derived DCs in the presence of a pan-retinoic acid receptor (RAR) inverse agonist greatly reduced IL22RA2v2 induction by Pam3CSK4 stimulation, revealing dependence of this induction on the retinoic acid biosynthesis pathway (Fig. 2.3, I-J).

We examined whether induction patterns of IL22RA2 observed in our myeloid cell models were consistent with expression in primary cells by stimulating CD14+ 
derived dendritic cells from healthy donors with Pam3CSK4 over 48 hours (Fig. 2.3K). Monocytes did not express IL22RA2 at 12 hours after stimulation, while IL22RA2v1 and IL22RA2v2 were detected at 24-48 hours. On the other hand, monocyte-derived dendritic cells expressed high basal levels of $I L 22 R A 2 v 1$ and IL22RA2v2 that decreased initially upon Pam3CSK4 stimulation, although recovery was achieved by 48 hours after stimulation. IL22RA2v3 was not detected in both cell types.

\subsection{DISCUSSION}

In this study, we identify the distinct properties of the IL-22BP isoforms and their effects on IL-22 signaling, alone and in concert with IL-17. While IL-22BPi2 and IL-22BPi3 differentially modulate IL-22 activity, IL-22BPi1 is retained intracellularly and inactive. We show that IL22BPi3 is the most abundantly expressed isoform in homeostatic conditions, while IL-22BPi2 alone is induced upon stimulation of myeloid cells. This study sheds light on the properties of human IL-22BP that are not reflected by murine models expressing only the IL-22BPi2 homolog.

We began by defining the biochemical characteristics and cellular localization of the IL22BP isoforms. Bioinformatics sequence analysis strongly predicts two N-linked glycosylation sites each for IL-22BPi1 and IL-22BPi2 and one site for IL-22BPi3 (Suppl. Fig. 2.1), suggesting that the double bands in IL-22BPi1 and IL-22BPi2 lysates arise from alternative glycosylation. Many cytokines and inteferons undergo glycosylation, which enhances their activity and stability. Indeed, IL-22 requires N-linked glycosylation for optimal binding to IL-10R2 ${ }^{183}$. We expect that the glycosylation of IL-22BP isoforms is similarly important to their function. Therefore, we used a eukaryotic Drosophila expression system to generate glycosylated recombinant IL-22BPi3 and accurately assess signaling effects. 
We found that IL-22BPi1 is retained intracellularly by exon 3, which is unique to this isoform. Since insertion of exon 3 into IL-22BPi3 is sufficient to prevent its secretion and ablate its suppressive capacity, lack of secretion is likely the principal reason why IL-22BPi1 fails to significantly alter IL-22 signaling. Previous evolutionary analysis found that exon 3 is a long terminal repeat retrotransposon introduced in the ape lineage ${ }^{170}$, which also explains the absence of IL-22BPi1 in rodents. Our data have now identified that a sequence in exon 3 changes the localization of IL-22BPi1 from that of its splice variants and prevents it from modulating IL-22 activity. Indeed, inclusion of exon 3 by alternative splicing may be a regulatory mechanism that diverts functional IL-22BPi2 or IL-22BPi3 expression to favor production of the inactive, intracellular isoform IL-22BPi1 instead. Also interesting is the location of exon 3 immediately upstream of Tyr-67 of IL-22BPi2 (Tyr99 of IL-22BPi1; Suppl. Fig. 2.1), which is alone responsible for four of six vital interactions between IL-22 and IL-22BPi2 ${ }^{99}$. Thus, exon 3 has the potential to alter conformation and affinity of the IL-22:IL-22BP bond. However, as IL-22BPi1 did not significantly alter IL-22 activity, any effects of exon 3 on the IL-22:IL-22BP interaction must be overridden by prevention of IL-22BPi1 release and hence opportunity to bind IL-22.

Our data from overexpression systems and recombinant proteins both present compelling evidence that IL-22BPi2 and IL-22BPi3 have differential antagonistic activity against IL-22 signaling. IL-22BPi2 comprises two fibronectin III domains ${ }^{99}$, while IL-22BPi3 lacks the Cterminus domain save for 5 frame-shifted residues (Suppl. Fig. 2.1). Prior structural analysis has shown that IL-22BPi2 interacts with IL-22 through 7 residues in its N-terminus fibronectin III domain and 3 residues in its C-terminus fibronectin III domain ${ }^{99,184}$. Retention of interactions in the N-terminus domain may explain the ability of IL-22BPi3 to antagonize IL-22 signaling, while the absence of the $\mathrm{C}$-terminus interactions likely causes its weaker strength of blockade compared 
to IL-22BPi2. Together, our signaling data show that IL-22 signaling can be inhibited by both IL22BPi2 and IL-22BPi3. However, the relative ability for the isoforms to inhibit IL-22 is distinct, creating two levels of blockade. This reflects the different binding kinetics shown by a previous surface plasmon resonance (SPR) study, which found the dissociation constant for IL-22BPi2 with IL-22 a thousand fold lower than that for IL-22BPi $3^{169}$. Notably, we found an IC $_{50}$ shift of 27.36 between IL-22BPi2 and IL-22BPi3 using a STAT3-luciferase reporter for biological IL-22dependent activation. The different ratios found between SPR and our luciferase assay may be due to different sources of recombinant protein; unlike proteins from the Drosophila expression system we used, recombinant protein from bacterial sources are not glycosylated and may therefore have different binding properties. They may also reflect the different temperatures at which measurements were performed or competition with the membrane-bound receptor IL-22R1 in a biological setting. While SPR provides insight into the bimolecular interaction, our readout of downstream signaling quantifies the biological function of IL-22BPi2 and IL-22BPi3 and therefore more closely reflects physiological effects. Differential blockade of IL-22 signaling extends to its synergy with IL-17, such that IL-22BPi2 and IL-22BPi3 skew the IL-17/IL-22 axis to different degrees in Th17 environments: complete blockade achieved by IL-22BPi2 favors expression of IL-17-dependent genes, while partial blockade by IL-22BPi3 retains co-operative actions between IL-22 and IL-17. Therefore, the composition of IL-22BP isoforms in an immune microenvironment can have a profound effect on the expression profile of inflammatory genes associated with barrier immunity. During these investigations, we also documented a loss of detection of IL-22 protein by ELISA in the presence of IL-22BPi2 but not IL-22BPi3 (Suppl. Fig. 2.6). Less sensitive detection of IL-22 in the presence of IL-22BPi2 might be due to the blockade of epitopes at the interface. Poor binding of detection or capture antibodies to IL-22 due to 
interference by IL-22BPi2 may be one explanation why IL-22 has proven difficult to detect in clinical samples by ELISA.

While quantifying expression of IL22RA2 isoforms in healthy human tissues, we found the first clues that $I L 22 R A 2$ isoforms are independently regulated. Previous studies have carried out semi-quantitative expression analyses of IL22RA2v1 and IL22RA2v2, but not IL22RA2v3, in human tissues ${ }^{98,157-160}$. Our expression data for IL22RA2v1 and IL22RA2v2 concur with previous observations in the placenta, stomach, spleen and thymus ${ }^{98,157,185}$; while significant IL22RA2v2 expression has also been reported in mammary gland and lung, we did not see this in our samples. This was surprising as we expected $I L 22 R A 2 v 2$ to be the major isoform based on previous studies, which likely missed these differences by failing to examine IL22RA2v3 gene expression. Based on the differential biological activity of IL-22BPi2 and IL-22BPi3 against IL-22, we speculate that these isoforms provide spatial and temporal control of IL-22 activity during homeostasis and inflammation. Since expression levels of IL22RA2 isoforms do not correlate with those of the membrane-bound receptors for IL-22, IL-22BP expression may not necessarily reflect local paracrine signaling effects and that IL-22BP may have a more complex role modulating IL-22 signaling at a distal site.

Other groups have shown that myeloid cells are an important source of IL22RA2 transcripts ${ }^{95,96,161}$. We found that stimulation with retinoic acid, either directly or indirectly through upstream activation of TLR2, induced only IL22RA2v2 in three different models representing different major populations: THP-1 monocytes, dendritic cells derived from THP-1 and granulocytic neutrophils from HL-60. IL22RA2vI and IL22RA2v3 expression were much lower and largely unaltered in these models. We found that IL22RA2v2 was similarly the principal isoform expressed and modulated by TLR2 stimulation in primary monocytes and monocyte- 
derived DCs, while IL22RA2v3 was not detected. In primary monocytes, IL22RA2v1 and IL22RA2v2 were only induced late at 24-48 hours after Pam3CSK4 stimulation, but undetectable at 12 hours after activation; on the other hand, monocyte-derived DCs had high basal expression levels of these isoforms that decreased by 12 hours after stimulation, but recovered over the course of 48 hours. Our results in monocyte-derived DCs concur with previous findings that TLR4 and TLR9 stimulation of these cells results in loss of IL22RA2 expression ${ }^{161}$, while we are the first to observe that expression is increased in primary monocytes stimulated via TLR2. The differing kinetics of IL22RA2 expression in monocytes (late) and monocyte-derived DCs (early) may reflect different physiological niches they occupy. Since IL-18 can be induced downstream of TLR stimulation and contributes to the suppression of IL-22BP in mice ${ }^{96}$, differences in its maturation particularly by the NLRC4 inflammasome may contribute to discrepancies between our immortalized and primary monocyte-derived DCs. It is intriguing that $I L 22 R A 2 v 1$ is coexpressed with IL22RA2v2 in primary cells despite its inactivity; its expression may be one way in which levels of active IL-22BPi2 are controlled.

Combining our data on the functions of exogenous IL-22BP and the regulation of endogenous IL-22BP, we conclude that IL-22BPi2 and IL-22BPi3 are the dominant isoforms actively controlling IL-22 signaling in humans, while IL-22BPi1 is inactive as it is intracellular. Therefore, we propose a model where IL-22BPi2 and IL-22BPi3 collaborate to form an IL-22BP rheostat that tunes IL-22 activity (Suppl. Fig. 2.7). During homeostasis, the rheostat is set low with IL-22BPi3 as the principal isoform regulating IL-22 activity, allowing low level IL-22 signaling and synergy with IL-17 for barrier immunity. When TLR2 stimulation or retinoic acid biosynthesis signal infection or inflammation, expression levels of the much stronger antagonist IL-22BPi2 are either increased or decreased in various myeloid populations, causing much larger changes to IL-22 
signaling that determine IL-17 dominance in Th17 settings. This rheostat presents a novel method by which IL-22 signaling is stringently controlled along a gradient specifically in humans, with likely impact on systemic IL-22 activity. Our characterization of the context-specific expression of IL22RA2 isoforms and their differential modulation of IL-22 activity also adds crucial understanding of genetic associations between IL22RA2 and various inflammatory conditions, which could not otherwise have been identified by genome-wide associations which do not distinguish the contributions of splice variants. Among these, one single nucleotide polymorphism has been identified in the IL22RA2 3'UTR, although the authors failed to identify a regulatory element that affected $I L 22 R A 2$ expression ${ }^{186}$. Other polymorphisms identified in these genetic association studies are not in the open reading frame. Based on our data, it would be interesting to identify additional polymorphisms in linkage disequilibrium that could affect the splicing of IL22RA2 isoforms.

Altogether, our results open avenues to understanding how IL-22BP acts in concert with IL-22 to modulate inflammation, and begin to parse the complex picture of how the genetic association of IL22RA2 with various inflammatory diseases translates into functions of IL-22BP in patients.

\subsection{MATERIALS AND METHODS}

\section{Cell culture conditions.}

Mammalian cell lines for signaling studies. Caco-2 cells were grown in complete Dulbecco's modified Eagle’s medium (DMEM, Sigma, St. Louis, MO) containing 20 \% (v/v) heatinactivated fetal bovine serum (HI-FBS, Atlanta Biologicals, Norcross, GA) and 1X penicillinstreptomycin-glutamine (P/S/G, Mediatech, Manassas, VA), $1 \mathrm{X}$ non-essential amino acids 
(NEAA) and 1mM sodium pyruvate (Gibco, Carlsbad, CA). Huh7 hepatocytes were cultured in DMEM containing $10 \%$ HI-FBS and $1 \mathrm{X}$ P/S/G.

Myeloid cells for expression studies. THP-1 monocytes were cultured in complete RPMI containing $10 \%$ (v/v) HI-FBS, 1X P/S/G, 10 mM HEPES, 1X NEAA, 1 mM sodium pyruvate and $50 \mu \mathrm{M}$ 2-mercaptoethanol (Sigma). To obtain THP-1-derived myeloid dendritic cells, THP-1 cells were seeded at $0.25 \times 10^{6}$ cells $/ \mathrm{ml}$ in tissue culture (TC)-treated T25 or T75 tissue culture flasks (Thermo Scientific) and stimulated with 100 ng/ml GM-CSF and 100 ng/ml IL-4 (Shenandoah Biotechnology, Warwick, PA) over 6 days, with half the medium replaced on day 3. HL-60 cells were grown in Iscove's modified Eagle's Medium (Sigma) containing $20 \%$ (v/v) HI-FBS. To differentiate them into a neutrophil model, HL-60 cells were seeded at $0.25 \times 10^{6}$ cells $/ \mathrm{ml}$ in tissue culture-treated T25 or T75 tissue culture flasks in the presence of $1.25 \%$ (v/v) DMSO (Sigma) for 5 days. Medium was replaced on day 3 and cells were diluted to $0.5 \times 10^{6}$ cells $/ \mathrm{ml}$ to maintain their capacity for differentiation. Stimulations were performed with $100 \mathrm{ng} / \mathrm{ml}$ Pam3CSK4, 1 $\mu \mathrm{g} / \mathrm{ml}$ LPS-EB from E.coli 0111:B4 (Invivogen, San Diego, CA) and $100 \mathrm{mM}$ all-trans retinoic acid (ACROS Organics, NJ) in 12-well TC-treated plates.

Primary monocytes and DCs. Primary cells were thawed and cultured in RPMI 1640 containing $10 \%(\mathrm{v} / \mathrm{v})$ HI-FBS, 1X P/S/G, $25 \mathrm{mM}$ HEPES and additional $2 \mathrm{mM}$ glutamine. Positively-isolated primary $\mathrm{CD} 14^{+}$monocytes were purchased frozen from a vendor. Monocytes were thawed and seeded at $1 \times 10^{6} / \mathrm{ml}$ in T25 flasks for stimulations. To obtain monocyte-derived DCs, monocytes were seeded at $3 \times 10^{6} / \mathrm{ml}$ in 6-well plates and treated with $178 \mathrm{ng} / \mathrm{ml} \mathrm{GM-CSF}$, $30 \mathrm{ng} / \mathrm{ml} \mathrm{IL-4}$ and $50 \mu \mathrm{M}$ 2-mercaptoethanol for 7 days, with half the medium replaced on days 2 and 4. On day 5, monocyte-derived DCs were re-seeded at $1 \times 10^{6} / \mathrm{ml}$ in 6 -well plates for stimulations. 
Drosophila S2 cells for recombinant IL-22BPi3 expression. S2 cells (Life Technologies, Carlsbad, CA) were cultured in complete S2 medium containing Schneider's Drosophila Medium (Life Technologies), $10 \%$ (v/v) HI-FBS, 1X P/S/G and $20 \mu \mathrm{g} / \mathrm{mL}$ gentamycin (Amresco, Solon, $\mathrm{OH}$ ). Complete medium was replaced one day after transfection. Cells were selected with 25 $\mu \mathrm{g} / \mathrm{mL}$ blasticidin (Invivogen) in complete medium for one passage, and then permanently transferred into ExpressFive Serum-Free medium (Life Technologies) supplemented with 1X $\mathrm{P} / \mathrm{S} / \mathrm{G}, 20 \mu \mathrm{g} / \mathrm{ml}$ gentamycin and $25 \mu \mathrm{g} / \mathrm{ml}$ blasticidin. Cultures were grown at $28{ }^{\circ} \mathrm{C}$ in a humidified incubator without additional $\mathrm{CO}_{2}$.

RNA tissue samples. RNA tissue samples were obtained from the Human Total RNA Panel II and Human Mammary Gland Total RNA (Clontech, Mountain View, CA).

RNA extraction and quantitative PCR. RNA was extracted using either the RNEasy Mini kit (QIAgen, Valencia, CA) or the Nucleospin RNA kit (Macherey-Nagel, Bethlehem, PA) and cDNA synthesized using the Quantitect Reverse Transcriptase Kit (QIAgen) according to manufacturers' instructions. Samples were then amplified by qPCR using Taqman reagents on the ViiA7 qPCR system (Life Technologies). Gene expression levels for cell lines were normalized to HPRT or $G A P D H$, and those for human primary tissue or cellular RNA were normalized to $A C T B$ ( $\beta$-actin). Gene expression was measured by qPCR using CXCL5, DEFB4A, DMBT1, MUC1 and SOCS3 Taqman probes (Life Technologies). Specific qPCR probes for IL22RA2 isoforms were custommade (Integrated DNA Technologies) with the sequences listed in Supplementary Table S2. 
Transfections and stimulations. The IL-22BP isoforms were cloned on a pUNO backbone (Invivogen) under control of EF1-HTLV promoters and transfected using Xtremegene 9 (Roche, San Francisco, CA) or calcium phosphate transfection (Life Technologies) according to manufacturers' instructions. Additionally, IL-22BPi1, IL-22BPi3 and IL-22BPi3-ex3 were cloned into pC4-FV1E (Clontech) backbones under control of CMV promoters. Supernatants were harvested 24 hours after transfection and incubated for 1 hour in the presence of recombinant cytokines prior to stimulation.

Untransfected cells were serum-starved for 4-6 hours before medium was replaced with supernatants containing cytokines pre-incubated with overexpressed or recombinant IL-22BP. Cells were stimulated with 10 ng/ml IL-22 (Peprotech, Rocky Hill, NJ), 10 ng/ml IL-17 (Shenandoah Biotechnology) or both. Whole cell lysates were harvested after 15 min of stimulation for phospho-STAT3 immunoblotting and after 24 hours for gene induction. Luciferase reporter cells were transfected in 48 -well plates using Xtremegene with a constitutively active eGFP vector and a luciferase reporter with a STAT3-responsive promoter (pGL4.47, Promega, Madison, WI) 24 hours before serum starvation.

Purification of recombinant IL-22BPi3. S2 cells were stably co-transfected with plasmids encoding IL-22BPi3-Histag and a blasticidin resistance gene in a ratio of 19:1 using calcium phosphate. Cell cultures were expanded in serum-free medium under constant blasticidin selection to a $500 \mathrm{ml}$ culture suspended at 125 r.p.m. When density exceeded 5.0 x $10^{6}$ cells $/ \mathrm{ml}$, we induced IL-22BPi3 expression with $0.8 \mathrm{mM} \mathrm{CuSO}_{4}$ over 8 days. Recombinant IL-22BPi3 was isolated from the filtered supernatant by affinity chromatography onto $\mathrm{Ni}^{2+}-\mathrm{NTA}$ resin (Thermo Pierce, Rockford, IL) and eluted in an imidazole (Sigma) gradient. Enriched eluate fractions were 
identified by SimplyBlue Safestain (Life Technologies), concentrated by ultracentrifugation and desalted by dialysis in PBS. Single-use aliquots were frozen in $20 \%$ glycerol and $0.1 \%$ BSA.

Coomassie staining and immunoblotting. 15-30 $\mu \mathrm{g}$ of cell lysates, $20 \mu \mathrm{L}$ of untreated supernatant, or $1 \mathrm{ml}$ of supernatant concentrated by TCA (Sigma) was subjected to SDS-PAGE and transferred to PVDF membranes (Thermo Scientific, Waltham, MA). After blocking in $5 \%$ (w/v) non-fat milk dissolved in Tris buffer saline containing Tween-20 (Sigma), staining was performed in either $5 \%$ milk or $5 \%$ bovine serum albumin (BSA, Sigma) for phospho-STAT3 (Tyr705), total STAT3, $\beta$-actin (13E5) (Cell Signaling, Danvers, MA) or IL-22BP (R\&D Systems, Minneapolis, MN). Coomassie staining was performed on SDS-PAGE gels with SimplyBlue SafeStain and destained overnight in $20 \%(\mathrm{w} / \mathrm{v}) \mathrm{NaCl}$.

ELISA assays. ELISA was performed using the IL-22 Quantikine kit (R\&D Systems) and the IL22 LEGEND MAX kit (Biolegend, San Diego, CA) according to the manufacturers' instructions. Samples containing IL-22 only or IL-22 in the presence of rhIL-22BP were preincubated for 1 hour in PBS before application to assay plates.

Statistics. Statistics were performed for quantitative assays in GraphPad Prism using one-way analysis of variance (ANOVA) with multiple comparisons against IL-22 stimulated samples, except in Fig. 2.2H where comparisons are made against IL-17 and IL-22 co-stimulated samples. 


\section{$2.5 \quad$ FIGURES}

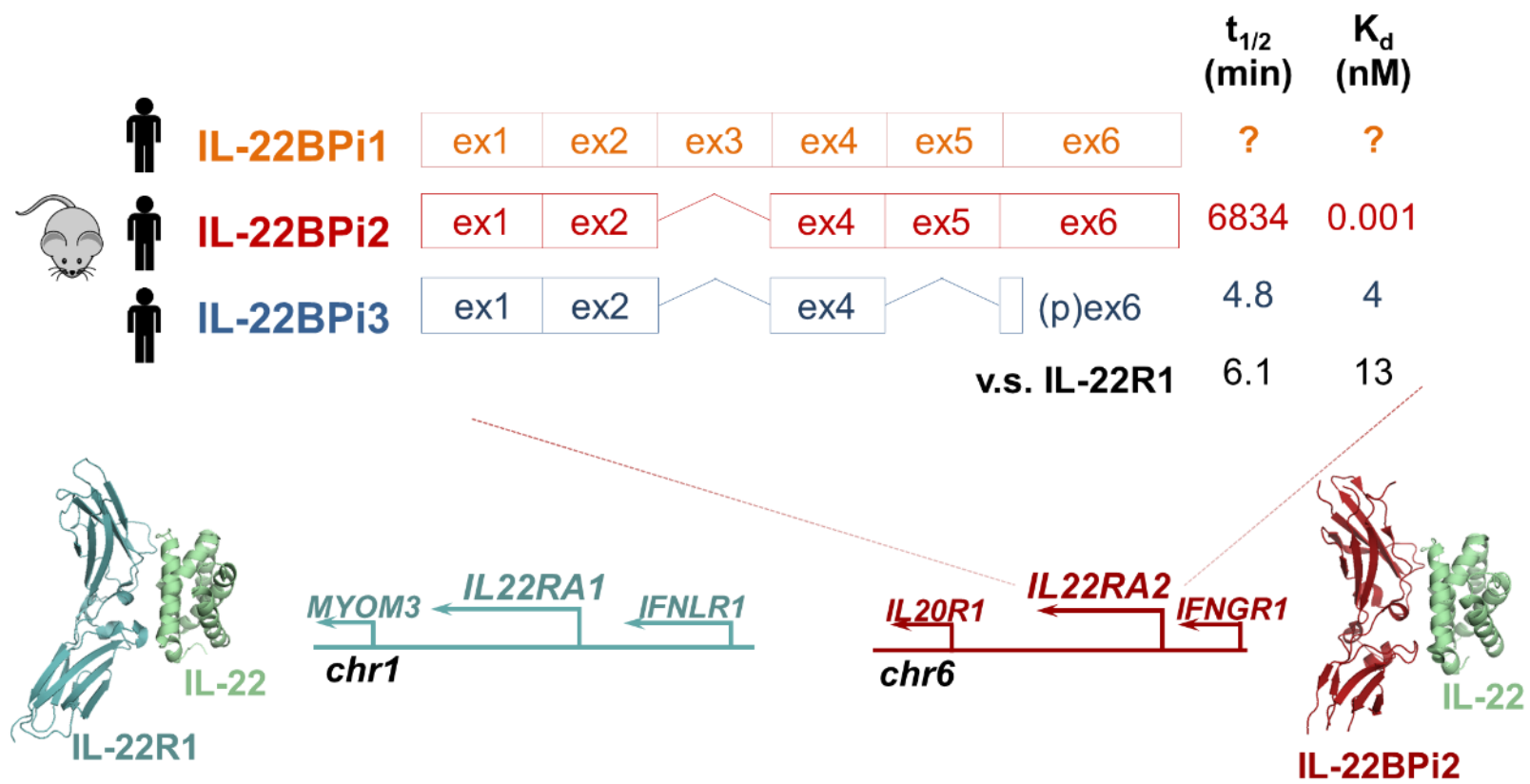

Figure 2.1. Known characteristics of IL-22 binding protein (IL-22BP) isoforms.

IL-22BP, encoded by IL22RA2, is a soluble receptor for IL-22 expressed as three alternatively spliced transcripts in the human, of which only IL-22BPi2 is found in mice. IL-22BPi2 and IL22BPi3 have different binding affinities for IL-22 determined by surface plasmon resonance. IL22BPi2 has a similar three-dimensional structure to the membrane bound receptor IL-22R1, which is encoded on a different gene and chromosome. The locations of IL22RAI and IL22RA2 on different chromosomes contrast many cytokine receptor soluble/transmembrane pairs, which are usually encoded on the same gene and generated by alternative splicing or enzymatic cleavage. 


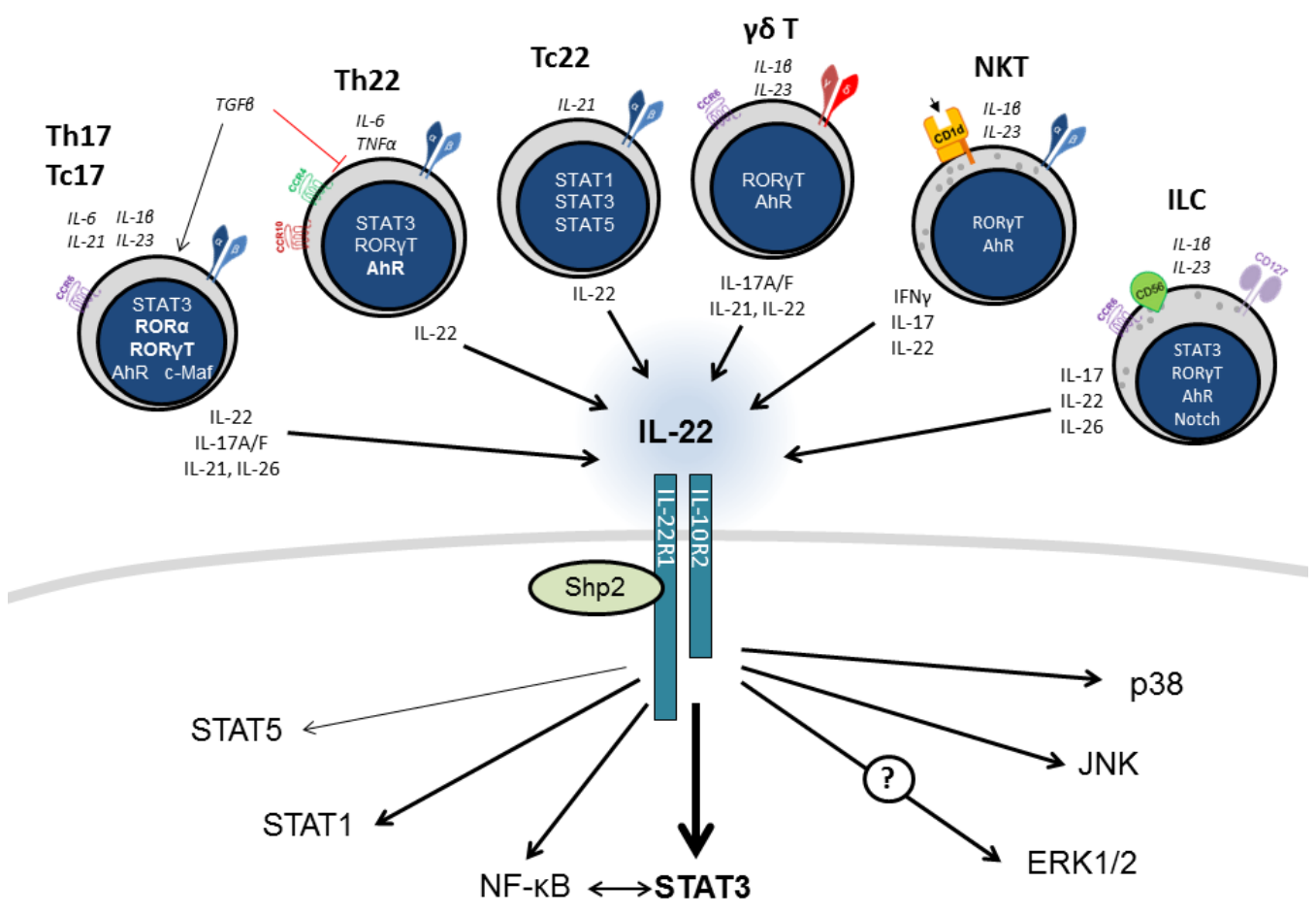

Figure 2.2. Sources of IL-22 and its principal downstream signaling pathways.

IL-22 is produced by several populations of lymphoid cells, largely under control of STAT3, $\mathrm{ROR} \alpha, \mathrm{ROR} \gamma \mathrm{T}$ and aryl hydrocarbon receptor (AhR). These activate signaling via the receptor comprising IL-22R1 and IL-10R2 that is expressed exclusively on non-immune cells under normal conditions, including hepatocytes, keratinocytes and epithelial cells of the colon and lung. Principal functions of IL-22 signaling are mediated through STAT3 activation, although activation of STATs 1 and 5, MAP kinases, NFאB, p38 and JNK have also been reported. STAT3 constitutively associates with IL-22R1 and rapidly responds to phosphorylation of IL22R1 by Shp2 following IL-22 stimulation. 
A

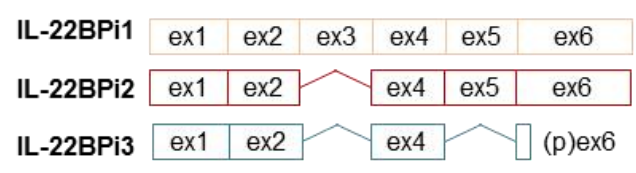

B

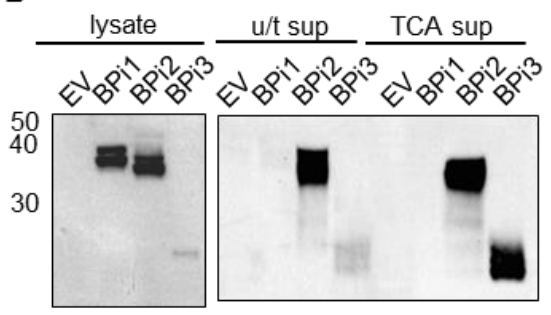

C

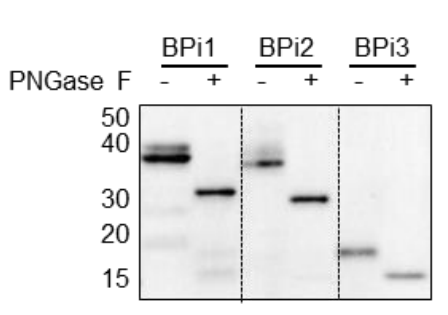

D

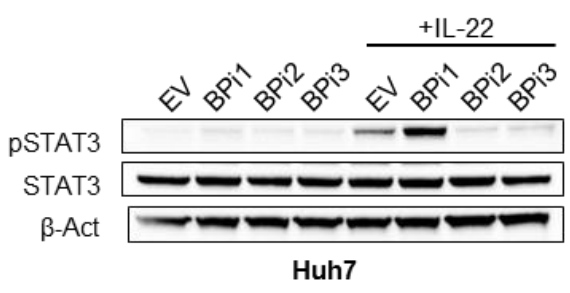

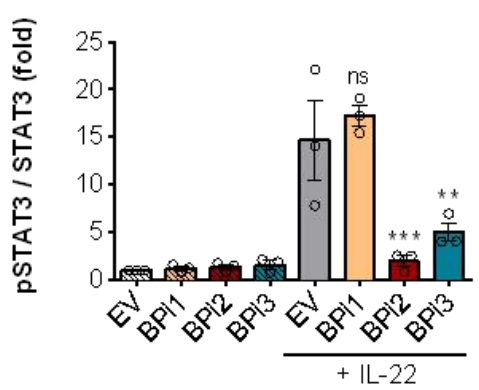

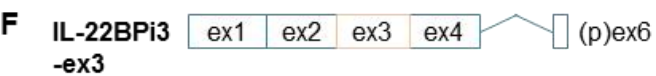

E

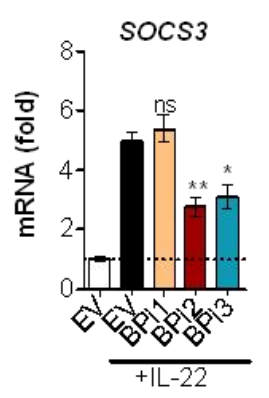

G

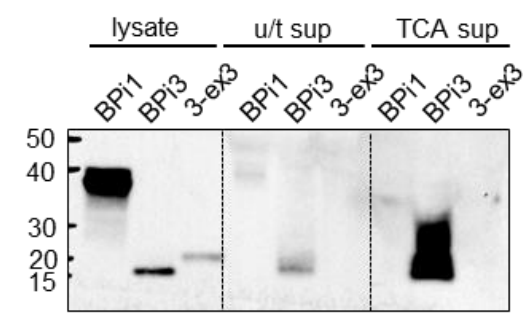

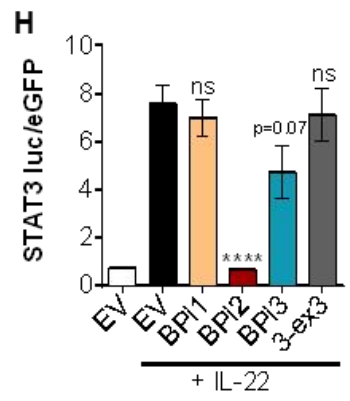

Figure 2.3. IL-22BP isoforms differentially modulate IL-22 activity when overexpressed.

(A) Schematic showing exon usage in the coding sequences of IL-22BPi1, IL-22BPi2 and IL22BPi3 generated by alternative splicing. (B) Immunoblot of IL-22BPi1, IL-22BPi2 and IL22BPi3 in transfected Huh7 cell lysate, untreated cell culture supernatant and supernatant concentrated by trichloroacetic acid (TCA). (C) Immunoblot of lysates from IL-22BP isoformoverexpressing cells treated with Peptide-N-Glycosidase F (PNGase F) to determine their Nlinked glycosylation. (D) Effects of overexpressed IL-22BP isoforms on IL-22 signaling measured by immunoblot of STAT3 phosphorylation 15 min post-incubation in hepatocyte cell line Huh7, with total STAT3 and $\beta$-actin used as loading controls for normalization for densitometry. (E) Effects of overexpressed IL-22BP isoforms on IL-22 signaling measured by qPCR of SOCS3 in Huh7 cells 24 hours post-incubation, normalized to endogenous control $H P R T$. (F) Schematic showing IL-22BPi3-ex3 protein created by sub-cloning exon 3 from IL22BPi1 into the open reading frame of IL-22BPi3. (G) Immunoblot of IL-22BPi1, IL-22BPi3 and IL-22BPi3-ex3 in transfected Huh7 lysate, untreated supernatant and TCA-treated supernatant. (H) Effect of overexpressed IL-22BPi1, IL-22BPi3 and IL-22BPi3-ex3 in supernatants on IL-22 activity 6 hours post-incubation in Huh7 cells, measured by luciferase reporter activity downstream of a STAT3-responsive promoter. (B,D,E,G,H) Data are 
representative of 2-3 independent experiments and presented as mean \pm s.e.m. ns $=$ not significant, $* \mathrm{p}<0.05, * * \mathrm{p}<0.01, * * * \mathrm{p}<0.001$ 
A

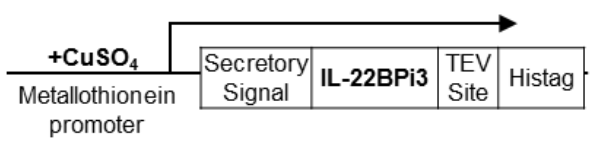

B

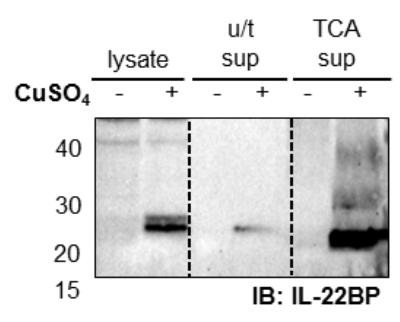

C

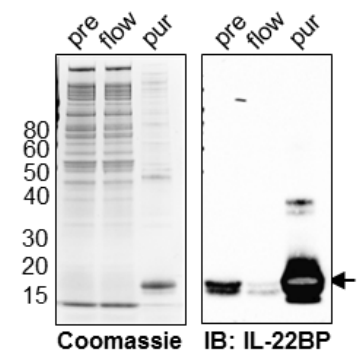

E

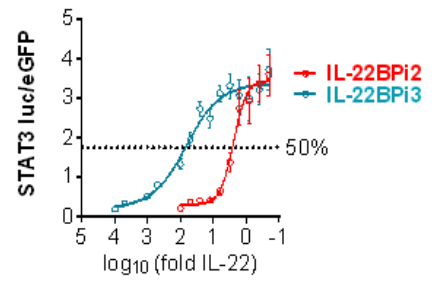

$\log _{10}$ (fold IL-22)
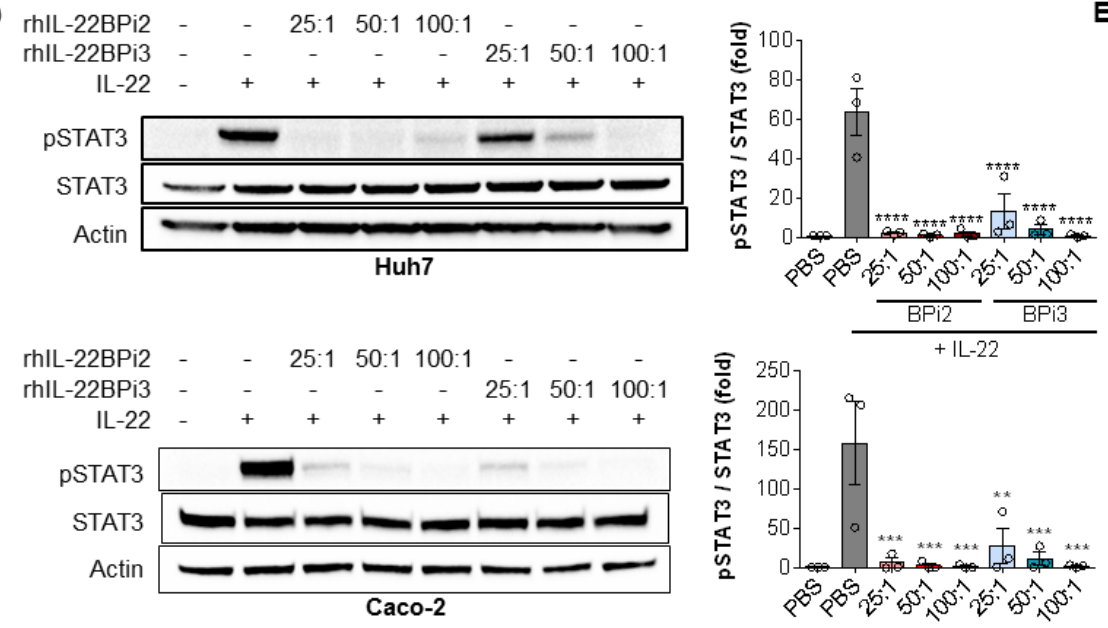

D rhlL-22BPi2 rhlL-22BPi3

PSTAT3

STAT3

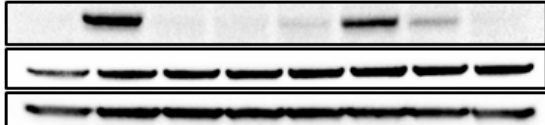

Huh7
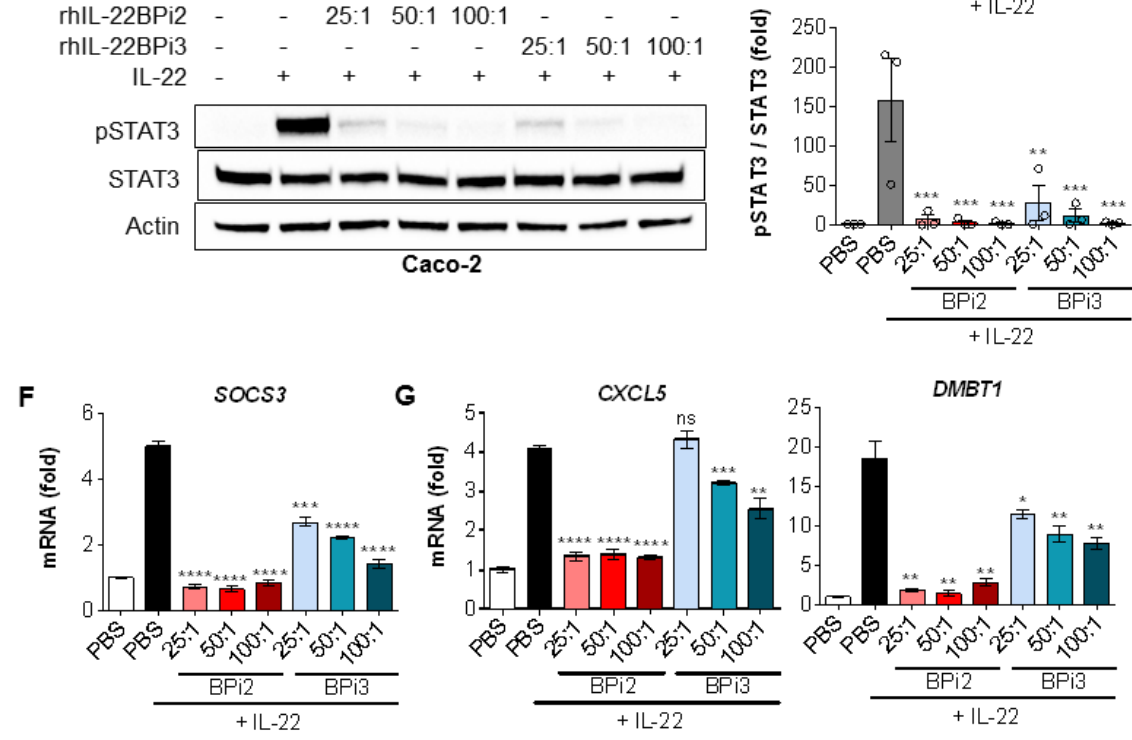

G
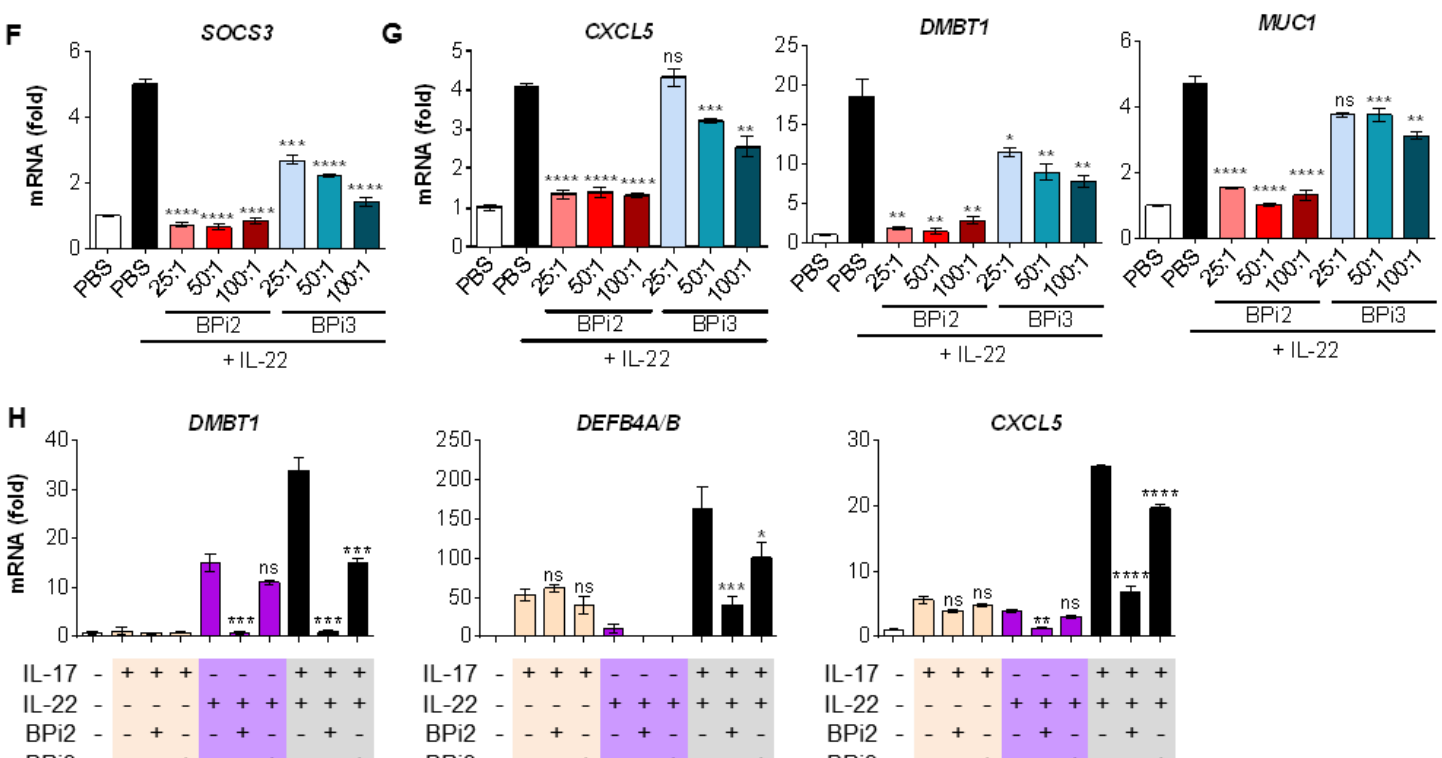

$\mathrm{BPi} 2$

BPi3

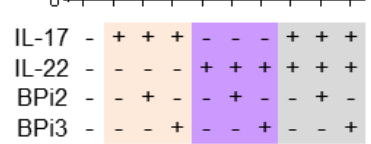

Figure 2.4. Recombinant human IL-22BPi2 and IL-22BPi3 proteins modulate IL-22 activity.

(A) Schematic showing IL-22BPi3-encoding construct with a $6 x H i$ tag driven by a metallothionein promoter, which was stably transfected into an S2 Drosophila expression system for secretion. (B) Immunoblot of IL-22BPi3 in lysate and supernatant of S2 cells induced by 
copper (II) sulfate. (C) Detection of IL-22BPi3 before and after isolation via $\mathrm{Ni}^{2+}$-NTA affinity chromatography by Coomassie stain and immunoblot. (D) Effects of increasing doses of recombinant IL-22BPi2 or IL-22BPi3 on IL-22 signaling in Huh7 hepatocytes and Caco-2 colonic epithelial cells measured by activation of STAT3 15 min post-incubation. Concentrations are expressed as the molar ratios of IL-22BP:IL-22. Total STAT3 and $\beta$-actin were used as loading controls for normalization in densitometry. (E) IC 50 curves of IL-22BPi2 and IL-22BPi3 for their blockade of IL-22 activity 6 hours post-co-incubation in Huh7 cells, measured by luciferase reporter activity downstream of a STAT3-responsive promoter. (F,G) Effects of increasing doses of recombinant IL-22BPi2 or IL-22BPi3 on induction of SOCS3 in Huh7 cells (F) and CXCL5, DMBT1 and MUC1 in Caco-2 cells (G) 24 hours after IL-22 stimulation. (H) Effect of 100-fold molar excess of IL-22BPi2 and IL-22BPi3 on synergistic induction of IL-22dependent $D M B T 1$, IL-17-dependent $D E F B 4 A / B$ and IL-17 or IL-22-inducible $C X C L 5$ in Caco-2 cells. Each IL-22BP isoform was applied to IL-17-only and IL-22-only stimulations as well as costimulations of IL-17 and IL-22. (F-H) HPRT used as endogenous control. (D-H) Data are representative of 2-4 independent experiments and are presented as mean \pm s.e.m. ns $=$ not significant, $* \mathrm{p}<0.05, * * \mathrm{p}<0.01, * * * \mathrm{p}<0.001, * * * * \mathrm{p}<0.0001$. 

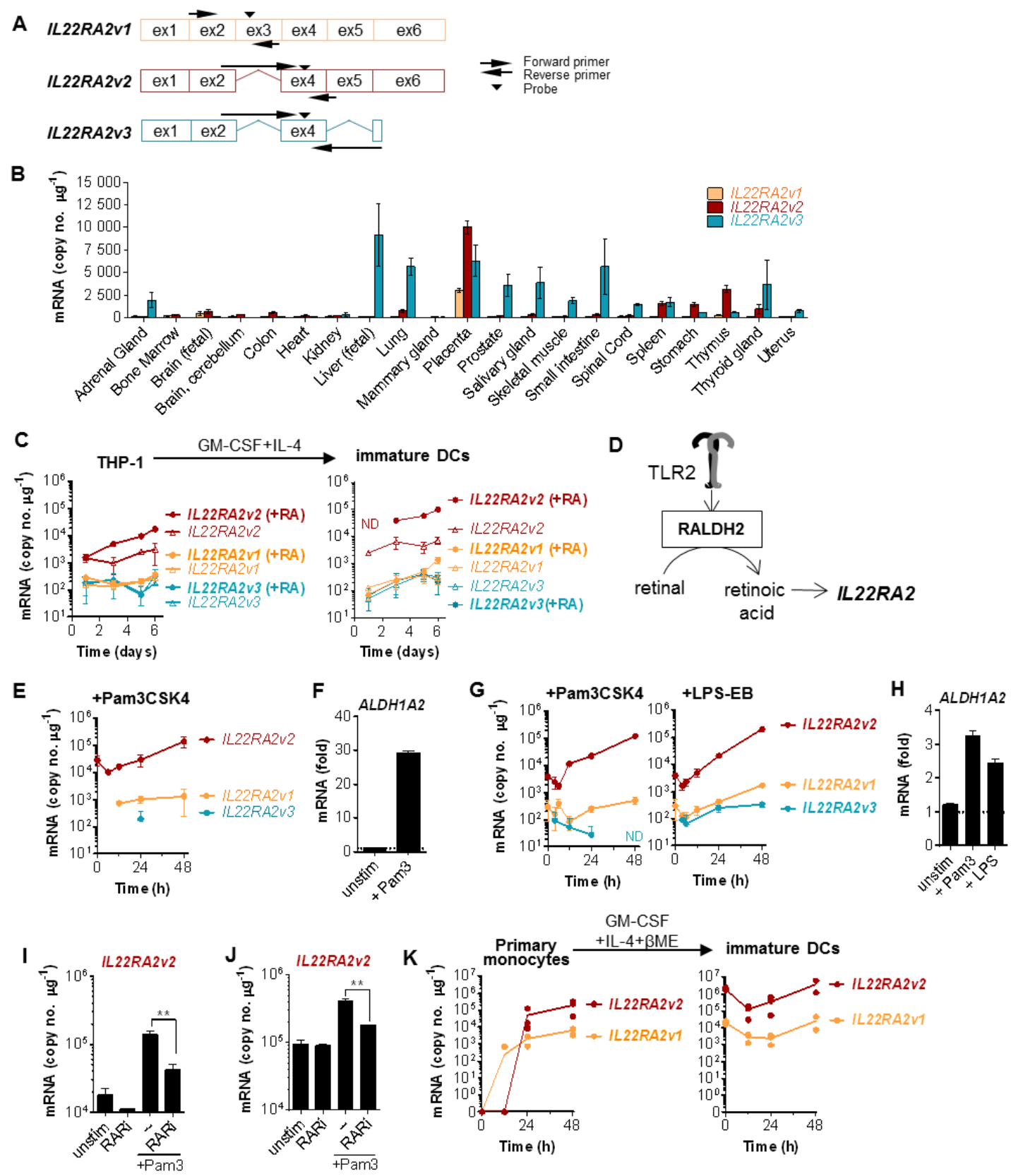

Figure 2.5. IL22RA2v3 is the most widely expressed isoform, while IL22RA2v2 is the principal isoform modulated via the TLR2-retinoic acid pathway.

(A) Schematic showing the gene structure encoding the three IL-22BP protein isoforms (IL22RA2v1, IL22RA2v2 and IL22RA2v3). Specific Taqman primers and probes that exploit the unique exon/intron configurations in each isoform are indicated by arrows.

(B) Expression of IL22RA2v1, IL22RA2v2 and IL22RA2v3 were measured and quantified by qPCR in 21 human tissues. Copy numbers of IL22RA2 isoforms were obtained from standard curves in Suppl. Fig. 2.3A. (C) THP-1 cells were used as undifferentiated monocytes or differentiated into immature DCs using GM-CSF and IL-4 over 6 days. Expression of each $I L 22 R A 2$ isoform was quantified by qPCR in the presence or absence of retinoic acid 
stimulation. (D) Schematic of pathway linking IL-22BP induction with TLR2 stimulation. TLR2 induces RALDH2 (ALDH1A2) expression and activity, increasing retinoic acid biosynthesis and in turn inducing IL22RA2 expression. (E,F) Undifferentiated THP-1 were stimulated with TLR2 ligand Pam3CSK4. (E) Expression of IL22RA2 isoforms was measured over 48 hours poststimulation. (F) Expression of ALDH1A2 by qPCR 12 hours post-stimulation. (G,H) THP-1derived DCs were stimulated via TLR2 with Pam3CSK4 and LPS-EB. (G) Expression of IL22RA2 isoforms measured over 48 hours post-stimulation. (H) Expression of ALDH1A2 12 hours post-stimulation. (I, J) Undifferentiated THP-1 cells (I) and THP-1-derived DCs (J) were stimulated with Pam3CSK4 in the presence or absence of pan-RAR inverse agonist BMS 493 for 48 hours and IL22RA2v2 expression measured. (K) Primary CD14 ${ }^{+}$monocytes and monocytederived DCs were stimulated over 48 hours with Pam3CSK4 and expression of each IL22RA2 isoform was quantified. IL22RA2v3 was not detected. (F, H) HPRT was used as an endogenous control. (C, E-J) Data are representative of 2 experiments and presented as mean \pm s.e.m. (K) Data from three donors are displayed as individual symbols with connecting lines representing means. $* * \mathrm{p}<0.01$. ND, not detected. 


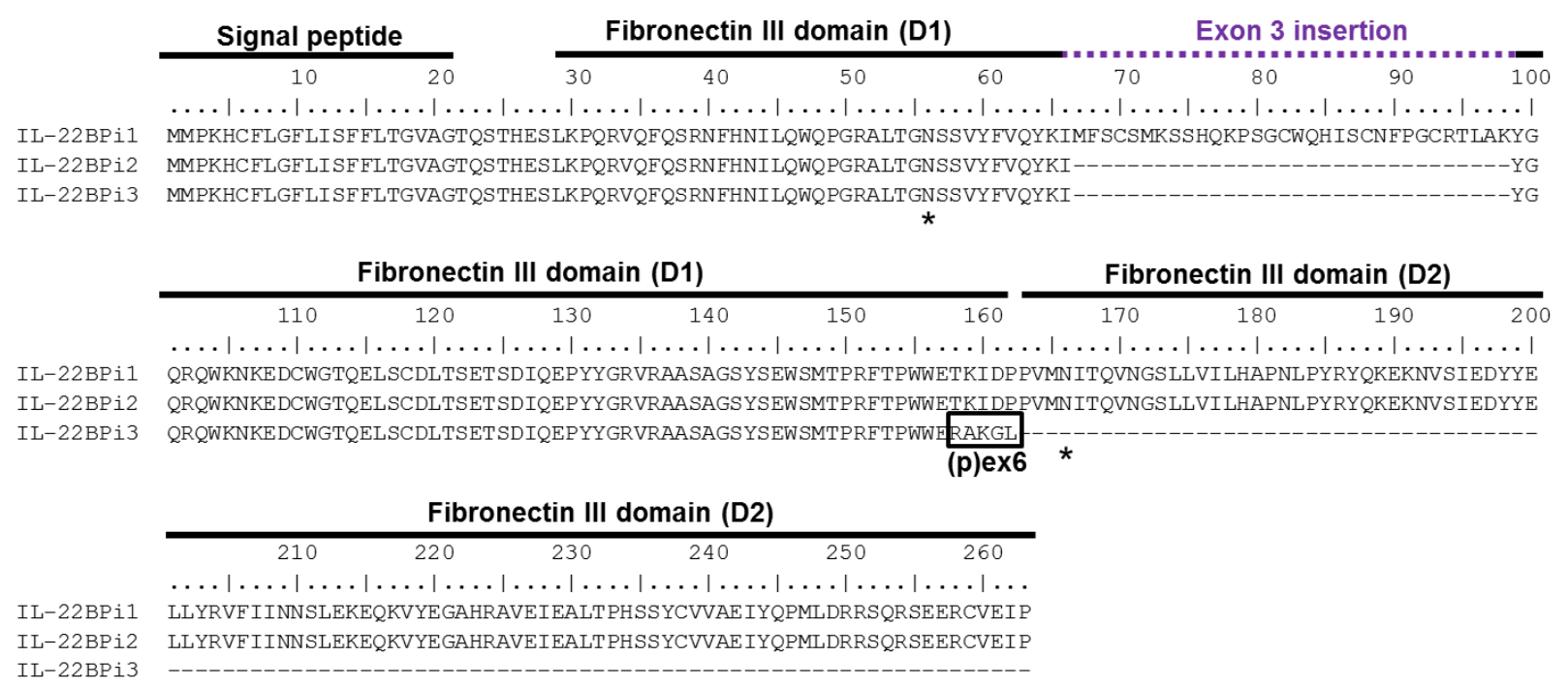

\section{Supplementary Figure 2.1. Alignment of IL-22BPi1, IL-22BPi2 and IL-22BPi3 protein sequences.}

Alignment was performed using CLUSTALW in BioEdit sequence alignment editor (http://www.mbio.ncsu.edu/bioedit/bioedit.html). Sequences are provided from NCBI (accessions NP_443194, NP_851827, NP_851826 respectively) and Uniprot (accession Q969J5). Major protein domains are annotated as in Uniprot and based on crystallography by de Moura et al. ${ }^{99}$. Asterisks indicate $\mathrm{N}$-glycosylation sites strongly predicted by the NetNGlyc 1.0 Server (http://www.cbs.dtu.dk/services/NetNGlyc/). 
A

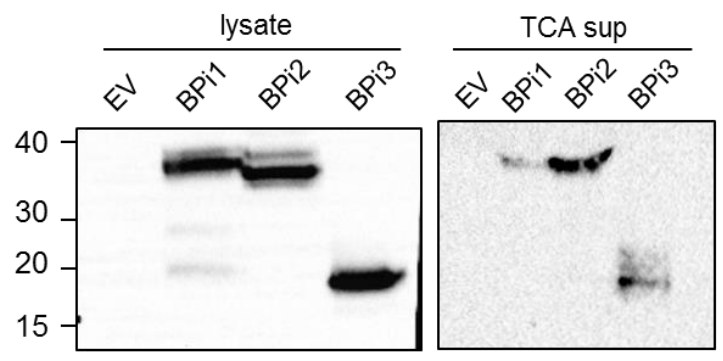

B

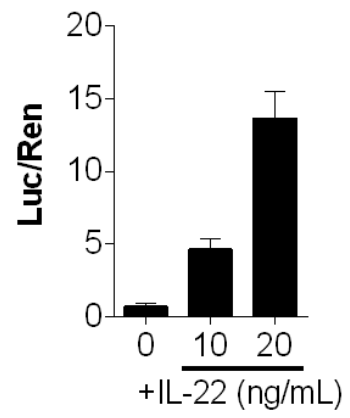

Supplementary Figure 2.1. Expression of IL-22BP isoforms in phospho-STAT3 immunoblot assays and optimization of dose for STAT3 luciferase reporter activity.

(A) Lysate and supernatant overexpression of IL-22BPi1, IL-22BPi2 and IL-22BPi3 in Huh7 cells $24 \mathrm{~h}$ after transfection, alongside empty vector $(\mathrm{EV})$ control. These correspond to the representative experiment in Fig. 1D. (B) Optimization of dose for STAT3 luciferase reporter activity 6 hours after IL-22 stimulation in HepG2 hepatocytes. Firefly luciferase activity was normalized against co-transfected Renilla luciferase activity. 
A
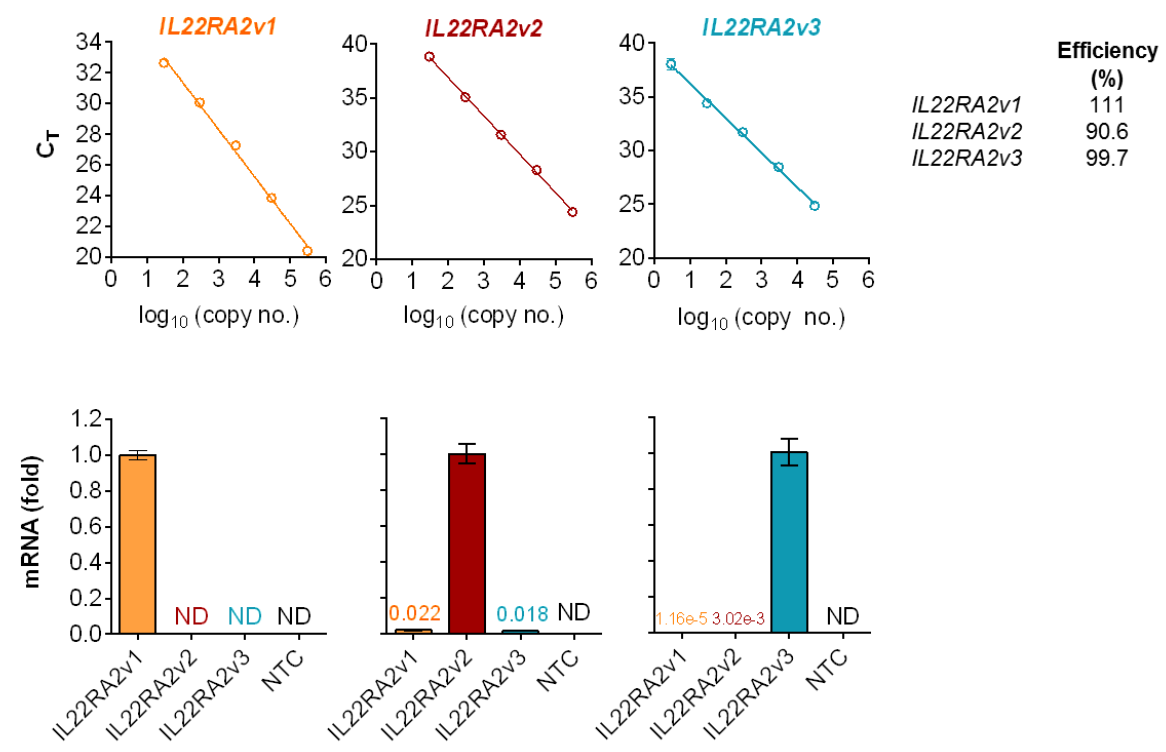

Supplementary Figure 2.2. Efficiency and specificity of the IL22RA2 Taqman probes.

(A) Standard curves were obtained by amplifying known copy numbers of vectors expressing each isoform of IL22RA2. Efficiency of each probe is provided in accompanying table. (B) $3 \mathrm{x}$ $10^{5}$ copies of expression vectors for IL22RA2v1, IL22RA2v2 and IL22RA2v3 were each amplified with all three sets of qPCR probes to verify their specificity for each isoform. 
A

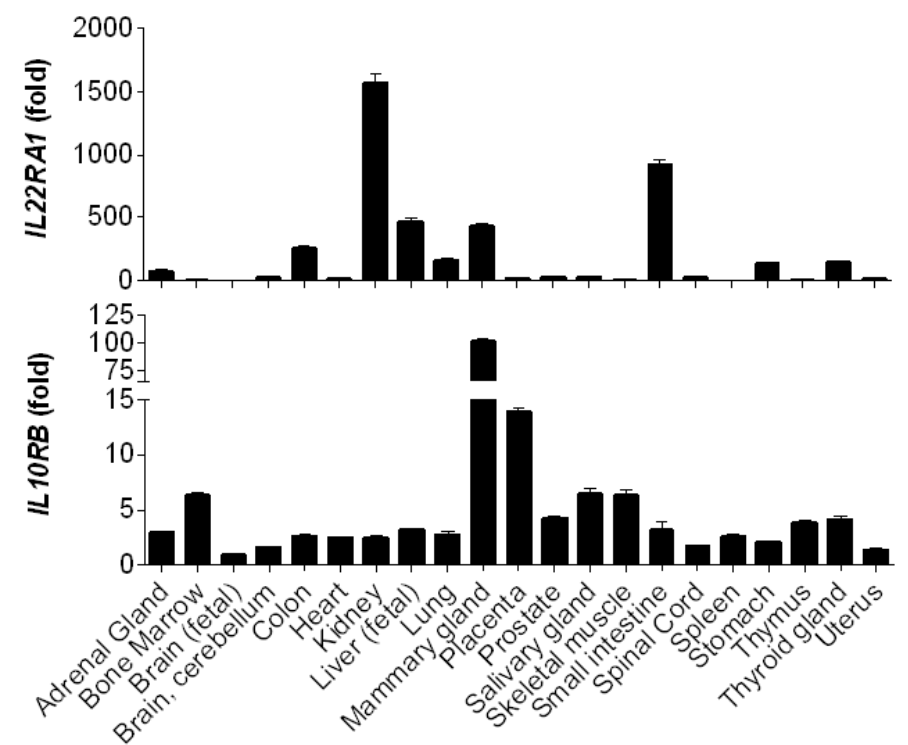

B

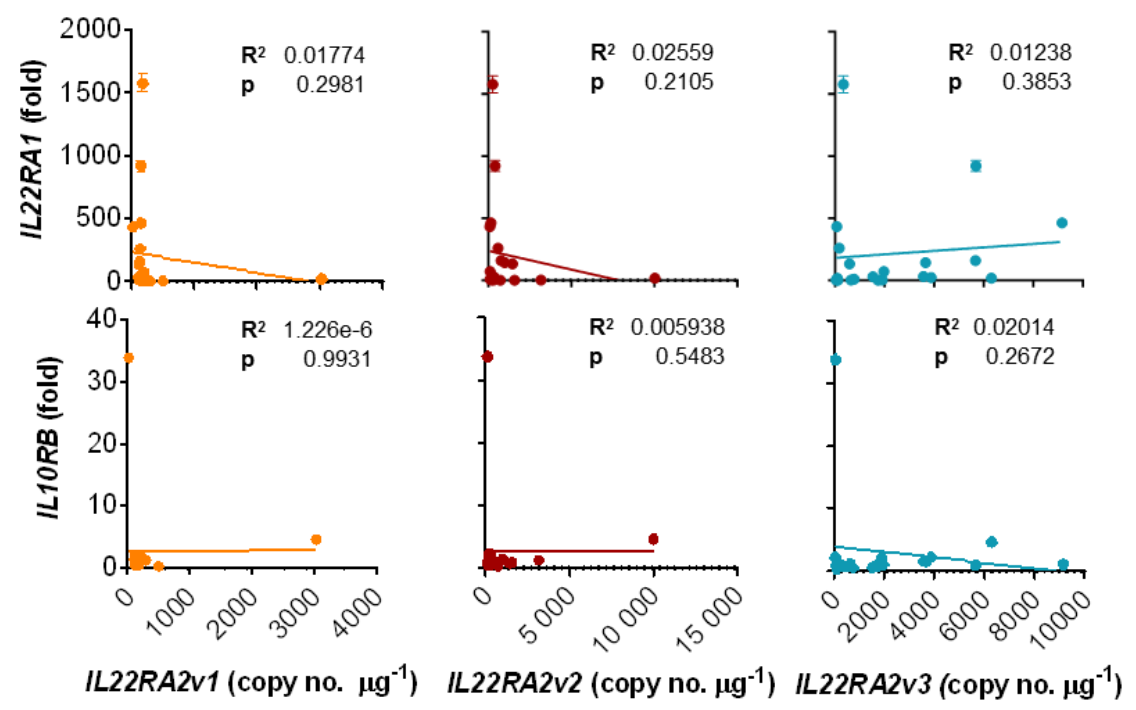

\section{Supplementary Figure 2.3. Correlation between expression levels of IL-22 receptor and IL22RA2 isoforms.}

(A) Expression of IL22RAI and ILIORB were measured by qPCR in 21 human tissues and plotted as fold difference from expression in fetal brain. ACTB was used as an endogenous control. (B) Linear regression analysis was performed to assess correlation between IL22RA2 isoform copy number and expression of $I L 22 R A 1$ or $I L 10 R B . \mathrm{R}^{2}$ and $\mathrm{p}$ (slope $\neq 0$ ) values are inset for each graph. ND, not detected. NTC, no template control. Data are represented as mean \pm s.e.m. 

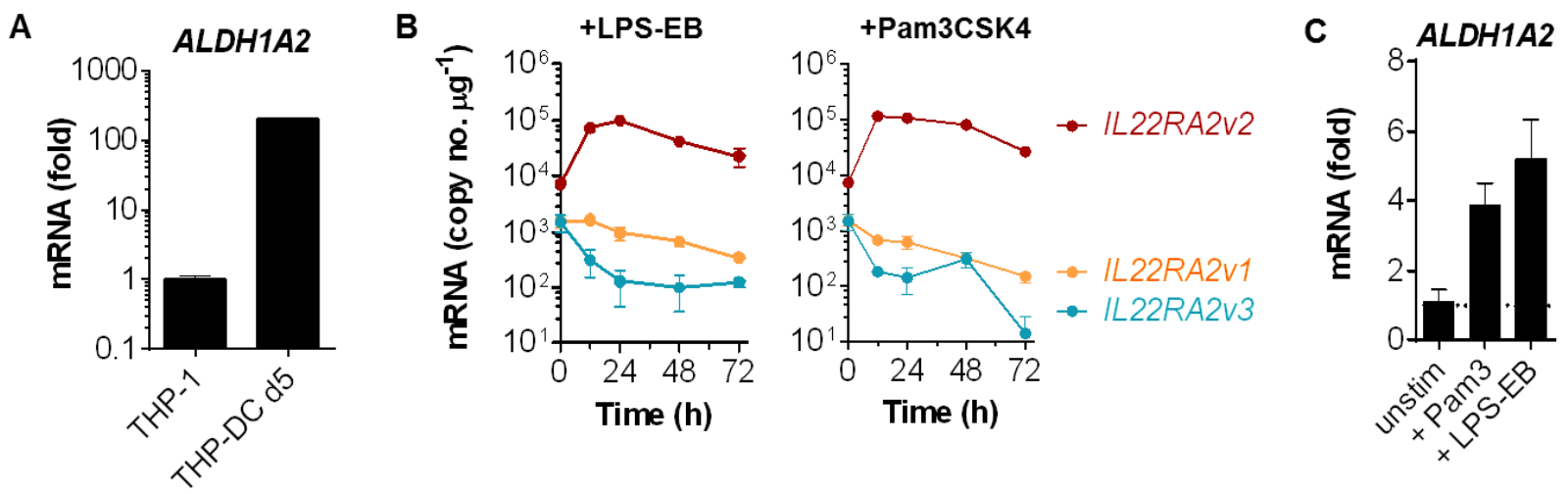

\section{Supplementary Figure 2.4. IL22RA2 and ALDH1A2 expression in HL-60-derived neutrophils, THP-1 and derivative DCs}

(A) Basal expression of ALDH1 A2 in undifferentiated THP-1 cells and THP-1-derived DCs after 5 days of differentiation. (B,C) HL-60 cells were differentiated over 5 days with DMSO into a neutrophil model and stimulated with Pam3CSK4 and LPS-EB. (B) Expression of IL22RA2 isoforms measured over 48h post-stimulation. (C) Expression of ALDH1A2 12h poststimulation.
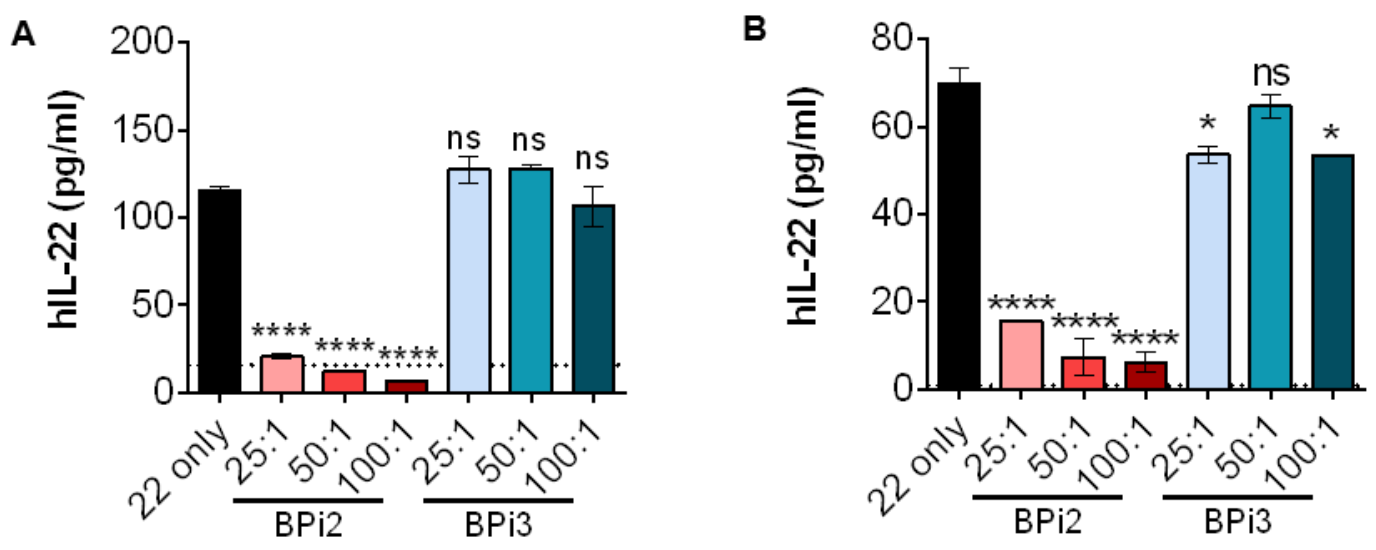

\section{Supplementary Figure 2.5. Effects of IL-22BPi2 and IL-22BPi3 on IL-22 ELISA.}

Detection of human IL-22 by ELISA after incubation with or without rhIL-22BPi2 or rhIL22BPi3 in varying molar ratios of IL-22BP:IL-22. Assays were performed using (A) R\&D's Quantikine ELISA kit and (B) Biolegend's LEGEND MAX ELISA kit for human IL-22. **** $\mathrm{p}$ $<0.0001,{ }^{*} \mathrm{p}<0.05, \mathrm{~ns}=$ not significant. 


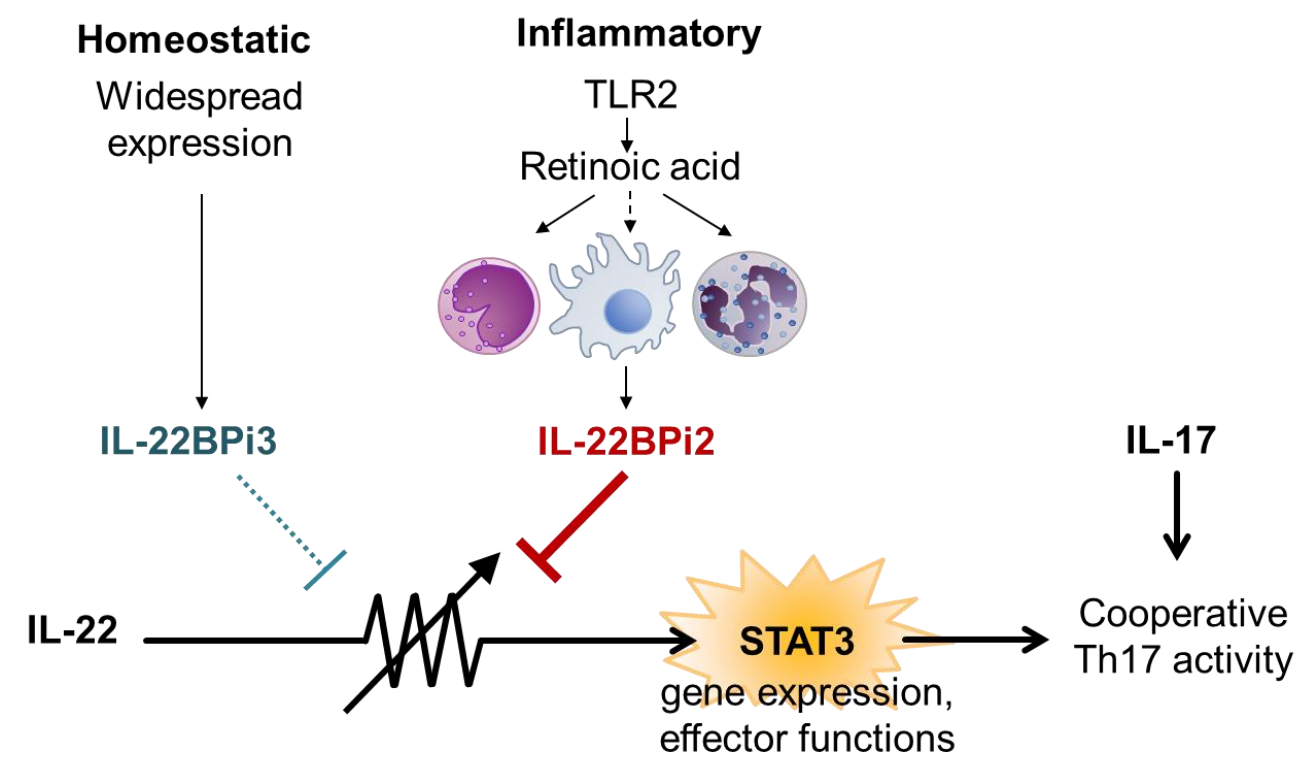

\section{Supplementary Figure 2.6. IL-22BPi2 and IL-22BPi3 collaborate to form a rheostat that tunes IL-22 activity.}

During homeostasis, IL-22BPi3 is widely expressed, preventing aberrant IL-22 signaling while allowing low level activation and synergy with IL-17 essential to important barrier immunity and regenerative functions. When inflammation activates the TLR2-retinoic acid pathway, only IL22BPi2 is modulated for its strong antagonism of IL-22 activity. The balance of isoform expression forms a gradient which controls STAT3 activation downstream of IL-22 activation and subsequent gene expression, effector functions and Th17 activity. 


\subsection{TABLES}

Table 2.1 Concentrations of rhIL-22BP used as 25:1, 50:1 and 100:1 excess of IL-22.

\begin{tabular}{|c|c|c|c|}
\hline $\begin{array}{l}\text { IL-22BP:IL-22 } \\
\text { ratio }\end{array}$ & $\begin{array}{l}\mathrm{IL}^{2} 22 \text { / } \mathrm{ng} \mathrm{ml}^{-1} \\
(16.5 \mathrm{kDa} \text { monomer })\end{array}$ & $\begin{array}{l}\text { rhIL-22BPi2-Fc / ng } \\
\mathrm{ml}^{-1}(51.3 \mathrm{kDa})\end{array}$ & $\begin{array}{l}\text { rhIL-22BPi3-Histag / ng } \\
\mathrm{ml}^{-1}(14.5 \mathrm{kDa})\end{array}$ \\
\hline $25: 1$ & 10.0 & 777.3 & 219.7 \\
\hline $50: 1$ & 10.0 & 1554.6 & 439.5 \\
\hline $100: 1$ & 10.0 & 3109.1 & 878.9 \\
\hline $25: 1$ & 20.0 & 1554.6 & 439.5 \\
\hline $50: 1$ & 20.0 & 3109.1 & 878.9 \\
\hline $100: 1$ & 20.0 & 6218.2 & 1757.9 \\
\hline
\end{tabular}

Table 2.2. Sequences of the qPCR probes and primers used to quantify IL22RA2 isoforms.

\begin{tabular}{|l|l|l|}
\hline Transcript & Primer & $5^{\prime}$ - Sequence - 3' $^{\prime}$ \\
\hline \multirow{3}{*}{ IL22RA2v1 } & Forward & ATTTTGCAATGGCAGCCTG \\
\cline { 2 - 3 } & Probe & TCTCACCAGAAGCCAAGTGGATGC \\
\cline { 2 - 3 } & Reverse & GCCTGGGAAGTTACAAGAAATG \\
\hline IL22RA2v2 & Forward & GTGCAGTACAAAATATATGGACAGA \\
\cline { 2 - 3 } & Probe & CTCGGCTGGGAGCTACTCAGAAT \\
\cline { 2 - 3 } & Reverse & GAGGATCTATTTTTGTTTCCCACC \\
\hline IL22RA2v3 & Forward & GTGCAGTACAAAATATATGGACAGA \\
\cline { 2 - 3 } & Probe & CTCGGCTGGGAGCTACTCAGAAT \\
\cline { 2 - 3 } & Reverse & CTTTTGCTCTTTCCCACCAG \\
\hline
\end{tabular}




\section{Chapter 3. EVOLUTIONARY ADAPTATIONS SUPPRESS INTERFERON LAMBDA 4 EXPRESSION DURING VIRAL INFECTION.}

Parts of the following work has been submitted for publication by the authors MeeAe Hong, Johannes Schwerk, Chrissie Lim, Alison Kell, Abigail Jarret, Joseph Pangallo, Yueh-Ming Loo, Shuanghu Liu, Curt Hagedorn, Michael Gale Jr. and Ram Savan. MeeAe Hong, Johannes Schwerk and Chrissie Lim equally contributed as co-first authors.

Interferon lambdas are critical antiviral effectors in hepatic and mucosal infections. While IFN $\lambda 1$, IFN $\lambda 2$ and IFN $\lambda 3$ act antiviral, genetic association studies have shown that expression of the recently discovered IFNL4 is detrimental to hepatitis C virus (HCV) infection through a yet unknown mechanism. Intriguingly, human IFNL4 harbors a genetic variant that introduces a premature stop codon. We performed a molecular and biochemical characterization of IFN $\lambda 4$ to determine its role and regulation of expression. We found that IFN $\lambda 4$ exhibits similar antiviral activity to IFN $\lambda 3$ without negatively affecting antiviral IFN activity or cell survival. We show that humans have evolved mechanisms to limit expression of functional IFN $\lambda 4$ through non-coding splice variants and non-functional protein isoforms. Furthermore, protein-coding IFNL4 mRNA are not loaded onto polyribosomes and lack a strong polyadenylation signal, resulting in poor translation efficiency. This study provides mechanistic evidence that humans have sustained adaptations to suppress IFN $\lambda 4$ expression, suggesting that immune function is dependent on other IFNL family members. 


\subsection{INTRODUCTION}

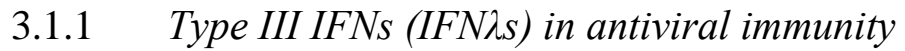

Interferons (IFNs) are cytokines central to innate immune responses. They have been categorized as type I (e.g. IFN- $\alpha, \beta, \omega)$, type II (IFN- $\gamma$ ) and type III (IFN- $\lambda$ ). Each category occupies a different niche in the immune response as they interact with distinct families of receptors on different cells, tissues and organs. The most recently discovered family, the type III IFNs, are encoded on in close proximity on the human IFN lambda (IFNL, IFN $\lambda$ ) locus on chromosome 19, composed of IFNL1 (IL29), IFNL2 (IL28A), IFNL3 (IL28B) and IFNL4 genes located on chromosome $19^{187}$. Of these members, IFN $\lambda 4$ has been most recently discovered and most studies to date characterize IFN $\lambda 1$, IFN $\lambda 2$ and IFN $\lambda 3$. These IFN $\lambda$ s signal through a heterodimeric receptor composed of IFN $\lambda$ R1 and IL-10R2 chains that activates the Jak/STAT pathway to induce IFN stimulated genes (ISGs) and antiviral activity. Among them, IFN $\lambda 3$ has the greatest antiviral activity, at 2-fold higher than IFN $\lambda 1$ and 16 -fold higher than IFN $\lambda 2{ }^{188,189}$.

While type I and III IFNs share many antiviral functions, including activation of signal transducer and activator of transcription (STAT)1, STAT2, IRF3, IRF7 and IRF9, the type I IFN receptor chains (IFNaR1 and IFN $\alpha$ R2) are nearly ubiquitously expressed while IFN $\lambda \mathrm{R} 1$ expression is limited to hepatocytes, epithelial cells of the lung, intestine, skin and cells of myeloid lineage so that the type III IFN response is far more tissue-restricted ${ }^{187,190,191}$. Other distinctions between the type I and III IFN pathways are slowly coming to light, including the strength of signaling, kinetics of activation and mediators of upstream induction. To begin, initial type III IFN activity is generally of lower strength and slightly delayed compared to type I IFN, but expression and activity are far more stable, making them the principal mediators of long-term antiviral activity ${ }^{192-195}$. Also, while the STAT, ISG and antiproliferative signatures activated by type I and III IFNs 
$\operatorname{are~highly~similar~}^{189,196-199}$, IFN $\lambda 3$ uniquely activates Janus kinase 2 (JAK2) for phosphorylation of STAT1 or $2^{200}$. Lastly, type I and III IFN induction via viral sensing by MAVS may occur through distinct pathways depending on where MAVS is localized, although opposing results have been found by different groups ${ }^{200,201}$. Thus type III IFNs have non-redundant functions from type I IFNs in antiviral immunity.

IFN $\lambda$-mediated immunity is essential to fight viral infections in the liver and at epithelial surfaces. Ifnlr $1^{-/-}$mice show that IFN $\lambda$ activity is required for antiviral protection against respiratory viruses including the influenza virus and the severe acute respiratory syndrome (SARS) coronavirus $^{202,203}$. In turn, human IFN $\lambda$ is the dominant IFN secreted by respiratory epithelial cells in response to influenza virus infection ${ }^{204,205}$ and is also produced by myeloid and lung epithelial cells during rhinovirus infection ${ }^{206}$. Similar to the respiratory tract, epithelial cells of the gastrointestinal tract are predominantly responsive to IFN $\lambda^{203,207}$, which initiates antiviral signaling critical for control of pathogenic enteric viruses ${ }^{207-209}$, rather than type I IFN. Uniquely among the IFN $\lambda$ s, IFN $\lambda 1$ is also expressed by T helper 17 (Th17) cells and is the only IFN associated with antiviral gene expression in psoriatic lesions ${ }^{210}$ Although these studies underscore the importance of IFN $\lambda s$ in antiviral immunity, the expression, regulation and activities of the individual members of the IFNL family during viral infection remain poorly understood.

Genome-wide association studies identified IFNL as a strong susceptibility locus for both natural and treatment-induced clearance of hepatitis C virus $(\mathrm{HCV})^{211-217}$. Of multiple SNPs identified as strong predictors, two major functional variants have been identified. The first, rs4803217, is a 3' UTR variant in IFNL3 identified by our group that dictates the stability and expression of the IFNL3 mRNA ${ }^{217,218}$. The second is a dinucleotide variant that controls the expression of functional IFNL4 and will be further discussed in the following sections. 


\subsubsection{IFNג4: Serendipitous discovery of a genetic paradox}

IFNL4 is a novel gene that was discovered, ironically, first by its genetic variant before its open reading frame. Prokunina-Olsson et al. performed an RNA screen by deep sequencing to find differential expression of transcripts in response to therapy in primary human hepatocytes and found strong association between a dinucleotide polymorphism ss469415590 (TT/ $\Delta \mathrm{G})$ and clearance of hepatitis $\mathrm{C}$ virus (HCV) infection ${ }^{216}$. Intriguingly, the variant occurred in the first exon of a novel open reading frame (ORF), named IFNL4 for its location within the IFNL locus, and determined whether a productive transcript was made. While the "TT" dinucleotide variant of IFNL4 creates a premature stop codon due to a frame shift that renders it a pseudogene, the ancestral " $\Delta G$ " single nucleotide variant encoded functional IFN $\lambda 4$ proteins of predicted sizes 179aa (full-length), 170aa, 131aa and 107aa (hereafter termed "IFN $\lambda 4$ p179", "IFN $\lambda 4 p 170 "$, "IFN $\lambda 4$ p131" and "IFN $\lambda 4$ p107" respectively). Surprisingly, the "TT" variant that prevents expression of functional IFNL4 associated with improved HCV clearance, while the " $\Delta \mathrm{G}$ " variant encoding the full-length protein associated with $\mathrm{HCV}$ persistence. The conclusion that IFN $\lambda 4$ ORF expression is pro-viral starkly contrasts the highly established, classical antiviral activity of all other known IFNs in many infections, especially for HCV which is treated traditionally with pegylated IFN- $\alpha$. Many studies have since replicated the strong IFNL4 association with HCV clearance/persistence ${ }^{218-223}$. Naturally, the robust genetic association and high odds ratios has prompted great academic interest in the potential mechanisms by which IFN $\lambda 4$ acts in a pro-viral manner, a major paradox for the IFN field which remains unexplained. Furthermore, the IFNL4 " $\Delta G$ " (risk) allele is in strong linkage with the risk allele for the functional IFNL3 variant at rs $4803217^{218}$, making the role of each variant particularly hard to distinguish. 


\subsubsection{Known characteristics and function of IFNג4}

Of the type II cytokines and interferons, IFN $\lambda 4$ shares the highest amino acid similarity and identity with the IFN lambdas, and highest with IFN $\lambda 3$ (similarity $40.8 \%$, identity $29.1 \%$ ) $^{216}$. Homology modelling shows that general folding of the cytokines and specific helices interacting with IFN $\lambda$ R 1 are expected to be conserved between IFN $\lambda 3$ and IFN $\lambda 4$, though amino acid differences enable and support formation of a disulphide bridge in IFN $\lambda 4$ that is absent in IFN $\lambda 3^{224}$.

Of the isoforms predicted in the original discovery, IFN $\lambda 4 \mathrm{p} 107$, IFN $\lambda 4 \mathrm{p} 131$ and IFN $\lambda 4$ p179 were verified and detectable by antibodies. Prokunina-Olsson et al. showed that overexpressed IFN $\lambda 4 p 179$ is able to activate a co-transfected interferon stimulatory response element (ISRE) luciferase reporter, while IFN $\lambda 4$ p107 and IFN $\lambda 4$ p131 did not induce any response $^{216}$. Several groups have gone on to examined gene induction by IFN $\lambda 4 \mathrm{p} 179$ and generally found it to be similar to IFN $\lambda 3$. RNA sequencing of HepG2 cells overexpressing IFN $\lambda 4 p 179$ revealed an enrichment mainly of pathways associated with canonical interferon responses and pattern recognition of bacteria or viruses, with some hits for the BRCA-mediated DNA damage response and IL-17 response. A side-by-side comparison later showed that both overexpressed and recombinant IFN $\lambda 4$ p179 activated an ISRE luciferase reporter, induced canonical interferonstimulated genes and increased clearance of $\mathrm{HCV}$ and coronavirus infection in vitro with equal afficacy as the same dose of IFN $\lambda 3^{224}$. A gene array also revealed similar classical antiviral gene induction in primary human hepatocytes and airway epithelial cells stimulated with recombinant IFN $\lambda 3$ or IFN $\lambda 4 p 179^{66}$. A recent study suggested that IFN $\lambda 4 p 179$ but not p131 overexpression may be able to induce cell death and reduce proliferation of stably transfected HepG2 hepatocytes, although the effect on mortality was comparatively modest and not compared alongside other $\mathrm{IFNs}^{225}$. Thus, the numerous similarities in signaling downstream of IFN $\lambda 4 \mathrm{p} 179$ and IFN $\lambda 3$ 
generally fail to explain their opposite genetic associations with viral clearance. While IFN $\lambda 4$ p107 and IFN $\lambda 4$ p131 remain poorly characterized, they have already demonstrated differential activity from IFN $\lambda 4$ p179 and could be responsible for non-canonical functions of IFN $\lambda 4$.

Meanwhile, poor detection of endogenous IFN $\lambda 4$ in primary samples poses obstacles to study of its activity and regulation. Consequently, some studies stratify patient cohorts and discern the effect of IFN $\lambda 4$ by whether it is "detectable" or "undetectable", being unable to perform twodimensional quantitative analyses ${ }^{226,227}$. This contrasts the ready detection of other type III IFNs in these studies and raises questions on the biological relevance of IFN $\lambda 4$ activity. Nonetheless, the expression of IFN $\lambda 4$ p107, p131 and p179 isoform transcripts has been recapitulated in liver samples, primary human hepatocytes and A549 lung carcinoma cells. Surprisingly, the majority of the transcripts cloned from primary samples were of the TT genotype that encodes a premature stop codon ${ }^{219}$. Analyses have also been performed examining correlations between genotype and markers of the antiviral state that alas extend the paradox: although carrying the $\Delta \mathrm{G}$ allele associates with poorer sustained virological response as expected, it still correlates with increased expression of important antiviral effector ISG15, supporting the transcriptomic profiles of overexpressed IFN $\lambda 4^{226,228}$.

An additional genetic polymorphism, rs117648444, encodes a P70S substitution in IFNL4 which attenuates its ability to induce antiviral ISGs. The variant encoding the less active S70 variant associates independently with improved both spontaneous clearance of HCV and response to pegylated IFN/ribavirin therapy ${ }^{228}$. Thus, it appears that IFN $\lambda 4$ may have already undergone other forms of intrinsic selection in its short evolutionary history. 


\subsubsection{Research objectives on IFNג4 and its isoforms}

While genetic association between IFNL4 and response to treatment of $\mathrm{HCV}$ is clear, its mechanism of action remains unclear. Similarities between overexpressed full-length IFN $\lambda 4 p 179$ and the canonical type III IFN IFN $\lambda 3$ fail to explain the paradoxical association between IFN $\lambda 4$ expression and poor viral clearance. Furthermore, several studies have failed to detect secretion of IFN $\lambda 4$ protein, prompting speculation on non-canonical activities of IFN $\lambda 4$, including an intracellular role $216,219,229-231$. Again, genetic variation does not clearly implicate any particular isoform with the association; strong linkage disequilibrium with a functional IFNL3 genotype at rs 12979860 and rs4803217 further complicates efforts to distinguish the contributions of IFN $\lambda 3$ and IFN $\lambda 4$ to the striking disease phenotype ${ }^{218,232}$. Much remains to be elucidated of the function of these isoforms and the regulation of their expression, which are likely to provide important clues to the non-canonical biological activites of IFN $\lambda 4$ in viral infection.

In this study, we use molecular and biochemical approaches to show that IFN $\lambda 4$ has similar anti-viral activities as IFN $\lambda 3$ but is weakly induced and poorly translated during viral infection. Our investigation revealed that the lower expression of IFNL4 is due to host adaptation suppressing the functional full-length isoform (179aa) of IFNL4 through induction of alternative, nonfunctional, intron-retention splice forms and weak polyadenylation signal. This study provides clear mechanistic evidence that humans have sustained adaptations to suppress IFN $\lambda 4$ expression suggesting that antiviral function is dependent on other $I F N L$ family members based on functional and genetic studies. 


\subsection{RESULTS}

\subsubsection{Bioactivity of IFNג4 in comparison to IFNג3.}

Differential mRNA splicing of the IFNL4 gene produces three protein-coding isoforms termed IFNLAP107, IFNLAP131 and IFNLAP179 based on the number of amino acids encoded (Fig. 3.1A). To test their individual activities, we overexpressed IFN $\lambda 4 p 107$, IFN $\lambda 4 p 131$, IFN $\lambda 4$ p179 isoforms and IFN $\lambda 3$, all tagged with C-terminal hemagglutinin (HA) in Huh7 cells. Overexpression was verified by immunoblot using $\alpha$-HA and $\alpha$-IFN $\lambda 4$ antibodies (Fig. 3.1B). As the $\alpha$-IFN $\lambda 4$ antibody was raised against a peptide encoded in exon 2 , this antibody only detects IFN $\lambda 4$ p131 and IFN $\lambda 4$ p179 isoforms. However, $\alpha$-HA detected equal expression of all IFN $\lambda 4$ isoforms and IFN $\lambda 3$ in the whole cell lysate.

By immunoblotting, we detected two bands in the lysates for IFN $\lambda 4$ p131, IFN $\lambda 4$ p179 and IFN $\lambda 3$ (Fig. 3.1B). These bands usually arise from differential glycosylation, a post-translational modification that is coded for, and which a majority of IFNs and cytokines require for efficient secretion and stability. To test if the higher molecular weight bands reflect glycosylated forms of IFN $\lambda 4 p 131$, IFN $\lambda 4$ p179 and IFN $\lambda 3$, we treated the overexpression cell lysates with PNGase F and immunoblotted with $\alpha$-IFN $\lambda 4$ and $\alpha$-HA. We observed that the higher molecular weight band was reduced, indicating that IFN $\lambda 4$ p131 and IFN $\lambda 4$ p179 were glycosylated (Fig. 3.1C). While two bands were detected for IFN $\lambda 3$, PNGase F failed to reduce the higher molecular weight band, suggesting a non-N-glycosyl modification for IFN $\lambda 3$.

Interferons require secretion from the cell to engage with their cognate receptors at the cell surface and activate Jak/STAT signaling. To test if the IFN $\lambda 4$ isoforms were secreted into the supernatant, we performed immunoblots on supernatants, both before (neat) and after 
concentration by tricholoroacetic acid (TCA) precipitation (Fig. 3.1D). When IFNL isoforms were overexpressed, we documented secreted IFN $\lambda 4 \mathrm{p} 179$ and IFN $\lambda 3$ in both neat and TCA-treated supernatants. However, we did not detect IFN $\lambda 4$ p107 or IFN $\lambda 4$ p131 (Suppl. Fig. 3.1A). These data suggest that IFN $\lambda 4$ p179 and IFN $\lambda 3$ are released extracellularly, while IFN $\lambda 4$ p107 and IFN $\lambda 4$ p131 are retained intracellularly. The supernatants containing IFN $\lambda 4 p 179$ or IFN $\lambda 3$ proteins were then subjected to PNGase F treatment. The higher molecular weight band of IFN $\lambda 4 \mathrm{p} 179$ was reduced to a lower molecular weight suggesting that the secreted proteins are also glycosylated (Fig. 3.1E).

Another non-synonymous variant of IFNL4 (Pro70Ser; rs117648444) exists that changes Proline to Serine at position 70 of the IFN $\lambda 4$ protein ${ }^{216,228}$. This SNP results in lower activity of IFN $\lambda 4$, presumably due to changes in the protein structure, and has been associated with improved spontaneous HCV clearance and better treatment response in patients with " $\Delta \mathrm{G}$ " at rs 368234815 228. We also tested the secretion of IFN $\lambda 4$ p179 S70 (P70S) in comparison to IFN $\lambda 4$ p179 (P70) after expression of both variants in Huh7 cells and found that the S70 variant is secreted less efficiently (Suppl. Fig. 3.1A). Furthermore, expression of IFNL4P179 S70 (P70S) results in a lower interferon-stimulated gene (ISG) response compared to the IFNL4P179 P70 variant, as measured by MX1 qPCR (Suppl. Fig. 3.1B). Overall, in our overexpression system, the glycosylated form of IFN $\lambda 4$ p179 is efficiently secreted out of the cell, the P70 variant more than the P70S variant, while IFN $\lambda 4$ p107 and IFN $\lambda 4$ p131 are predominantly intracellular.

\subsubsection{IFNA4 signals exclusively through the extracellular IFNAR1:IL-10R2 receptor complex.}

Similar to other type III IFNs, IFN $\lambda 4$ is thought to signal through its cognate heterodimeric receptor composed of IFN $\lambda$ R 1 and IL-10R2 subunits ${ }^{224}$. Type III IFNs evolved from a common lineage with IL-10 family cytokines, many of which feature alternative receptor usage. Since 
IFNL4 shares this lineage and has low sequence identity with other type III IFNs ${ }^{216}$, we examined if IFN $\lambda 4$ could also signal through a different IL-10 family receptor. We co-expressed the IFNLA isoforms and IFNL3 together with a luciferase reporter downstream of an interferon stimulated response element (ISRE) in Huh7 wild-type and IFNLR $I^{-/-}$cells. We expressed the IFNL4 isoforms and IFNL3 in Huh7 cells. Cell supernatants were then transferred to Huh7 wild-type and IFNLRI ${ }^{-}$ I- cells expressing a luciferase reporter downstream of an interferon stimulated response element (ISRE). We found that expression of IFN $\lambda 4 p 179$, the only secreted isoform, and IFN $\lambda 3$ strongly induced ISRE luciferase reporter activity in wild-type Huh7 cells, whereas the non-secreted IFN $\lambda 4$ p107 and IFN $\lambda 4$ p131 were inactive (Fig. 3.1F). ISRE luciferase reporter activity was completely abrogated in $I F N L R 1^{-/-}$Huh7 cells, suggesting that IFN $\lambda$ R1 was necessary for ISG induction by IFN $\lambda 4$ p179. To further test if IFN $\lambda 4$ signals through extracellular IL-10R2, we blocked the receptor using a neutralizing $\alpha$ IL-10R 2 antibody and co-expressed IFN $\lambda 4$ p179 or IFN $\lambda 3$ together with the ISRE luciferase reporter. IL-10R2 blockade decreased ISRE luciferase reporter activity for both IFN $\lambda 4$ p 179 and IFN $\lambda 3$ (Fig. 3.1G). Lastly, downstream signaling via $M X 1$ was completely abrogated in IFNLRI $1^{-/}$cells upon stimulation with IFN $\lambda 4$ p179 (Fig. 3.1H). These data not only confirm that IFN $\lambda 4$ requires both IFN $\lambda$ R 1 and IL-10R 2 chains, but also shows that it signals through this extracellular heterodimeric receptor complex.

\subsubsection{Intracellular IFNג4 isoforms do not affect type I and III IFN signaling.}

As the majority of IFN $\lambda 4$ remain in the cytoplasm, including both functional and inactive isoforms, it has been proposed that intracellular IFN $\lambda 4$ regulates cell surface IFN $\lambda$ R 1 by binding and sequestering IFN $\lambda \mathrm{R} 1$ or IL-10R2 in the cytoplasm ${ }^{216,224}$. To quantify the effects of IFN $\lambda 4$ on receptor surface expression and consequent downstream signaling of other type III IFNs, we treated Huh7 cells overexpressing IFN $\lambda 4$ isoforms or an empty vector (EV) with recombinant 
human (rh)IFN $\lambda 3$ for $6 \mathrm{~h}$ and quantified ISG induction represented by $M X 1$. We found that induction of $M X 1$ by rhIFN $\lambda 3$ was unaltered in the presence of overexpressed IFN $\lambda 4 \mathrm{p} 107$ or IFN $\lambda 4$ p131 compared to EV transfection (Fig. 3.1I). Again, IFN $\lambda 4$ p179 alone was able to induce $M X 1$, and in this overexpression system it does so at similar levels compared with EV-transfected cells treated with $100 \mathrm{ng} / \mathrm{ml}$ rhIFN $\lambda 3$. To exclude that observed $M X 1$ expression is due to the nontransfected fraction of cells not expressing IFNL4 isoforms, we co-transfected a GFP plasmid together with the IFN $\lambda 4$ overexpression constructs and sorted for $\mathrm{GFP}^{+}$cells prior to qPCR analysis. Again, overexpression of IFN $\lambda 4 \mathrm{p} 107$ and IFN $\lambda 4 \mathrm{p} 131$ isoforms did not interfere with the cell's ability to respond to exogenous IFN $\lambda 3$ (Fig. 3.1J). We performed the same stimulation with IFN $\beta$ and found that overexpression of intracellular IFN $\lambda 4$ isoforms does not affect type I IFN signaling (Fig. 3.1K). These data show that IFN $\lambda 4$ p179 has a similar ability to induce ISGs as rhIFN $\lambda 3$ and that IFN $\lambda 4$ isoforms do not interfere with type I or type III IFN induced ISG responses intracellularly or extracellularly.

\subsubsection{Overexpressed IFNג4p179 and IFNג3 have comparable antiviral activity on HCV}

The IFN $\lambda 4$ polymorphism (rs368234815) is presumed functional because full-length IFN $\lambda 4$ protein coding potential encoded by the " $\Delta \mathrm{G}$ " genotype correlates with $\mathrm{HCV}$ persistence 216. This paradoxical association has led to the hypothesis that IFN $\lambda 4$ may have non-canonical functions such as blockade of antiviral activity. To test this hypothesis, we overexpressed IFN $\lambda 4$ isoforms and IFN $\lambda 3$ in Huh7 cells and infected them with a Renilla-luciferase tagged HCV reporter virus ${ }^{233}$. We observed that IFN $\lambda 4 \mathrm{p} 179$ and IFN $\lambda 3$ mediated similar antiviral activity and comparably suppressed HCV replication, while the intracellular IFN $\lambda 4$ p107 and IFN $\lambda 4$ p131 isoforms were unable to block HCV replication (Fig. 3.1L). These data suggest that IFN $\lambda 4$ p179 
has antiviral activities on HCV comparable to IFN $\lambda 3$ when overexpressed and indeed appears to perform similar to type III IFNs in the context of antiviral defense.

\subsubsection{Antiviral activity of recombinant human IFNג4}

To confirm the observations made with our plasmid-based overexpression system, we purified recombinant human (rh) IFN $\lambda 4$ p179 (rhIFN $\lambda 4)$ protein using a Drosophila Schneider 2 (S2) cell expression system. We cloned the IFNL4P179 open reading frame with a C-terminal 6xHistidine tag into a construct under control of a copper (II) ion-inducible metallothionein promoter and transfected the expression plasmid into S2 cells. Upon induction by copper (II) sulfate, rhIFN $\lambda 4$ was secreted into the supernatant, collected for affinity purification on a nickel column, and further isolated by size exclusion chromatography (Fig. 3.2, A-C). We compared the activities of rhIFN $\lambda 3$ and rhIFN $\lambda 4$ by quantifying $M X 1$ induction as a functional read out in PH5CH8 hepatocytes. The induction pattern of $M X 1$ over several $\operatorname{logs}$ of IFN $\lambda$ concentration yielded an $\mathrm{EC}_{50}$ difference of 2.879 (Fig. 3.2D). Intriguingly, the largest differences in activity were seen at lower concentrations of IFN $\lambda$. These data suggest that IFN $\lambda 3$ exhibits activity that is marginally higher than that of IFN $\lambda 4$, although these differences are minimal compared to differences in activities between IFN $\lambda 3$ and IFN $\lambda 2 \square^{188}$. We further tested the specificity for downstream STAT signaling in wildtype or IFNLR1 $1^{-/}$PH5CH8 cells (Suppl. Fig. 3.2) stimulated with $\operatorname{rhIFN} \beta$, rhIFN $\lambda 3$, or rhIFN $\lambda 4$ for 15 min (Fig. 3.2E). Treatment with rhIFN $\beta$, rhIFN $\lambda 3$, or rhIFN $\lambda 4$ induced phosphorylation of STAT1 (pSTAT1) which was completely abrogated in IFNLR $1^{-/}$cells, when stimulated with rhIFN $\lambda 3$ or rhIFN $\lambda 4$. To confirm downstream gene expression, we treated wild-type or $I F N L R 1^{-/-}$ PH5CH8 cells with rhIFN $\beta$, rhIFN $\lambda 3$ or rhIFN $\lambda 4$ for $6 \mathrm{~h}$ and quantified the induction of ISGs by qPCR. MX1, OAS1 and ISG15 were induced by rhIFN 44 treatment at comparable levels to rhIFN $\lambda 3$ treatment. This induction was again abrogated in $I F N L R 1^{-/-}$cells (Fig. 3.2, F-H). Control 
treatment with rhIFN $\beta$ resulted in stronger activation of pSTAT1 and induction of ISGs regardless of the $I F N L R I^{-\%}$ status.

Since genetic association data show that individuals carrying the in-frame " $\Delta G$ " variant have increased risk of viral persistence, we examined whether IFN $\lambda 4$ administration had unique detrimental effects on the host that were independent of virus infection per se, for example causing increased cell death. To test if IFN $\lambda 4$ causes cell death, as another potential mechanism rendering IFN $\lambda 4$ expression detrimental to the host, $\mathrm{PH} 5 \mathrm{CH} 8$ cells were treated with either rhIFN $\lambda 4$ (100 $\mathrm{ng} / \mathrm{ml}), \operatorname{rhIFN} \lambda 3(100 \mathrm{ng} / \mathrm{ml})$, or actinomycin D (ActD, $10 \mu \mathrm{g} / \mathrm{ml})$. Cell death and confluence were assessed over $70 \mathrm{~h}$ using an IncuCyte imaging system. The cells were treated in the presence of Sytox green, a dye which enters dying cells as they lose membrane integrity. Neither IFN $\lambda 3$ nor IFN $\lambda 4$ induced cell death, and cell viability was comparable to mock-treated cells (Fig. 3.2I). ActD treated cells served as a positive control for cell death in these assays and $M X 1$ induction was assessed as a control to show similar IFN $\lambda$ activities (Fig. 3.2J). When measured for cell confluence over time as a readout for proliferation, we found that IFN $\lambda 3$ and IFN $\lambda 4$ treatment shows a similar antiproliferative effect on cells compared to control cells (Fig. 3.2I). These data suggest that IFN $\lambda 4$ does not induce cell death in hepatocytes, which contradicts a previous report that found IFN $\lambda 4$ could induce cell death ${ }^{225}$. The authors of that study observed differences in the endoplasmic reticulum stress response and cell death in HepG2 cells using a plasmid-based overexpression system; this is in contrast to our use of titrated, recombinant IFN $\lambda$ proteins, which are likely a cleaner measure of cell death responses that would occur in vivo.

To test if the induction of antiviral ISGs by rhIFN $\lambda 4$ translates into functional suppression of viral propagation, as we had observed with the use of our plasmid-based overexpression system, we measured replication of HCV (Fig. 3.3A) and West Nile virus (WNV) (Fig. 3.3B) in the 
presence of rhIFN $\lambda 3$ or rhIFN $\lambda 4$. We documented robust antiviral activity against these viruses with rhIFN $\lambda 4$ treatment, which was comparable to that exerted by rhIFN $\lambda 3$ (Fig. 3.3, A-B). Comparable gene induction of 37 ISGs was observed in WNV infection post rhIFN $\lambda 3$ or rhIFN $\lambda 4$ treatments (Fig. 3.3C). These data confirm observations from our overexpression studies and conclusively show that rhIFN $\lambda 4$ displays antiviral activity comparable to that of IFN $\lambda 3$.

\subsubsection{IFNL4 is expressed at basal levels during viral infection.}

Previous studies have identified transcripts of the protein-coding isoforms IFNLAP107, IFNL4P131 and IFNL4P179 harboring the " $\triangle \mathrm{G}$ " allele ${ }^{216}$. As the induction pattern of IFNL4 isoforms during infection is not well documented, we cloned and generated a cDNA library from HepG2 hepatocytes (heterozygotes at rs368234815) stimulated with a RIG-I ligand (HCV 5'ppp RNA) or poly(I:C). Using primers flanking the coding region of IFNLAP179, we identified three additional IFNL4 transcripts with intron retention, which have a similar exon configuration to IFNL4P107, IFNLAP131 and IFNL4P179 (Fig. 3.4, A-B; Suppl. Fig. 3.3). We also identified pseudogenes with premature stop codons carrying the "TT" allele (Suppl. Fig. 3.3). Genes with intron retentions are not exported to the cytoplasm from the nucleus, or are subjected to nonsensemediated decay in the rare event export occurs.

To evaluate the induction patterns of these IFNL4 splice forms by qPCR, we designed primers specific to each protein-coding transcript as well as a specific primer pair that detects all IFNL4 isoforms with intron retention (IFNL4 IR). We established standard curves for these probes and for IFNL3 to measure absolute copy numbers for each isoform allowing to directly compare abundance across the transcripts (Table 3.1). Analysis of Huh7 cells (TT/ $\Delta \mathrm{G})$, HepG2 cells (TT/ $\Delta \mathrm{G})$, HeLa cells $(\Delta \mathrm{G} / \Delta \mathrm{G})$ and HEK293 cells $(\Delta \mathrm{G} / \Delta \mathrm{G})$ stimulated with the RIG-I ligand HCV 5'ppp RNA (HCV PAMP) and primary human hepatocytes (PHH; TT/ $\Delta \mathrm{G}$ or $\Delta \mathrm{G} / \Delta \mathrm{G})$ stimulated 
with poly(I:C) revealed that IFNL3 was highly induced compared to all the IFNLA transcripts (Fig. 3.4, C-F, I-J). Surprisingly, the next most abundant transcripts were IFNL4 with retained introns, rather than any of the protein-coding isoforms. These were followed by IFNLAP131, IFNL4P179 and IFNL4P107 isoforms. As dendritic cells have been reported to express IFNL1-3 during viral infection ${ }^{234,235}$, we also stimulated myeloid DCs differentiated from MUTZ-3 cells (TT/ $\left.\Delta \mathrm{G}\right)$ and THP-1 cells (TT/ $\Delta \mathrm{G})$ with poly(I:C) and examined for $I F N L$ expression patterns (Fig. 3.4, G-H). While poly(I:C) induced high IFNL3 expression in these DCs, the protein-coding IFNL4 isoforms were not induced, while the intron-retaining IFNL4 transcripts were observed only in THP-1derived DCs. We found that similar expression patterns, including low level IFNL4P179 expression, were seen in hepatoma cells during infections with WNV, Dengue virus (DenV), and Sendai virus (SeV) (Fig. 3.4, K-M). Our data reveal that IFNL4 is poorly induced compared to IFNL3 during viral PAMP stimulation and viral infection, and that amongst the IFNL4 isoforms the intron-retaining isoforms destined for nonsense-mediated decay are preferentially transcribed instead of the protein-coding isoforms.

\subsubsection{Endogenous IFNL4 isoforms are not translated efficiently during infection}

The poor induction of functional IFNL4P179 compared to IFNL3 during viral PAMP stimulation and viral infections would reflect low secretory output of the IFN $\lambda 4$ p 179 protein. To test this, we infected Huh7 and HepG2 cells with HCV, WNV and SeV, and the cell lysates and supernatants were immunoblotted for endogenous IFN $\lambda 4$ protein. In conformity with previous reports, cell lysates, direct cell culture supernatants and TCA-concentrated supernatants did not yield detectable IFN $\lambda 4$ protein. To ensure that this was not due to poor antibody sensitivity, we performed polysome fractionation to determine efficiency of active translation of IFNL3 and IFNL4 isoforms. Cell lysates from HepG2 cells stimulated with poly(I:C) or mock treated for 12 
$\mathrm{h}$ were separated using a sucrose gradient to separate non-translating monosome fractions of low centrifugal weight and polysome fractions of high centrifugal weight containing actively translated mRNA (Fig. 3.5, A-D). Polysome fractions were then pooled and IFNL3 and IFNL4 isoforms were quantified by qPCR. Intriguingly, IFNL4 isoforms were poorly detected (less than 40 copies) in the heavy polysome and non-translated monosome fractions, despite detection of high levels of IFNL3 in these fractions (Fig. 3.5, C-D), suggesting that IFNL4 isoforms have only low association with actively translating ribosomal fractions compared to IFNL3. We propose two main reasons for inefficient translation of IFNL4 mRNA. First, multiple isoforms of IFNLA, including intron-retaining variants, are induced upon viral infection or stimulation with viral PAMP. Expression of multiple splice variants, especially non-protein-coding transcripts, reduces transcriptional and translational resources for expression of the only functionally active IFNLAP179 isoform. Second, the 3' UTR of IFNL4, unlike other IFNL genes, does not harbor a canonical polyadenylation (polyA) signal (Fig. 3.5E). For mRNA termination, a polyA signal (canonical motif: AAUAAA) is essential for downstream cleavage and polyadenylation of mRNA. Polyadenylation signal sequences are not only critical for mRNA termination, but also for recruitment of RNA binding proteins essential for stability and subsequent translation. In silico analysis of the IFNL4 3' UTR did not yield a strong canonical polyA signal compared to the other IFNL genes (Suppl. Fig. 3.4). To test the polyA signal usage and to identify the downstream cleavage site (CS) essential for IFNL4 mRNA termination, we employed 3' Rapid Amplification

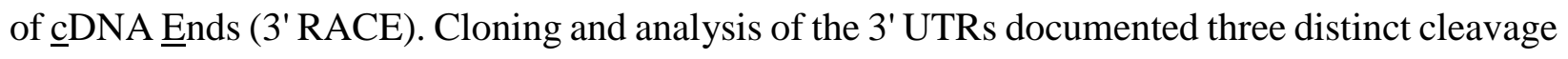
sites used by IFNL4 mRNA for termination (Fig. 3.5E). More importantly, 70.6\% of the analyzed sequences used the second cleavage site (CS\#2) followed by CS\#3 (23.5\%) and CS\#1 (5.9\%). Intriguingly, the 5' ends of CS\#2 (major cleavage site) and CS\#1 (minor cleavage site) do not 
encode for a canonical or non-canonical polyA signal. Only a small percentage of IFNL4 mRNA sequences use CS\#3, where a weak non-canonical polyA signal (AUUAAA) was detected, suggesting that this is not the major termination site. Previous studies have shown that eukaryotic mRNA do not tolerate changes in these nucleotide motifs and the efficiency of mRNA termination, polyadenylation and translation are severely hampered ${ }^{236}$. Therefore, we propose that the majority of IFNL4 mRNA that is not efficiently terminated, is rapidly degraded and weakly translated. Overall, basal low-levels of in-frame IFNL4 mRNA expression and poor translation lead to lack of IFN $\lambda 4$ protein expression.

\subsubsection{Non-functional IFNL4 splice variants arose before the $\Delta G>T T$ frame-shift variant in humans.}

Our studies suggest that IFN $\lambda 4$ mediates comparable antiviral activity to IFN $\lambda 3$, but its action is limited through mechanisms including poor endogenous expression, expression of nonfunctional alternative splice variants, isoforms with intron retention, a frame-shift mutation that begets a premature stop codon $(\Delta \mathrm{G}>\mathrm{TT})$, and the absence of a canonical polyA signal. As nonhuman primates do not carry the $\Delta \mathrm{G}>\mathrm{TT}$ frame-shift mutation and therefore have the potential to express full-length IFNL4, it is possible that humans have evolved multiple strategies for limiting the production of IFN $\lambda 4$ for yet unknown reasons. We hypothesized that if IFN $\lambda 4$ was detrimental to the host, we would observe such selection in non-human primates. Therefore, we stimulated Gorilla gorilla fibroblasts with poly(I:C) and found that they also expressed non-functional IFNLAP107 and IFNLAP131 isoforms, as well as unstable splice variants with intron retention (Fig. 3.5F). Like in humans, expression levels of functional IFNL4P179 were low. These data suggest that the expression of functional IFNLAP179 isoform has been selected against in nonhuman primates even before the dinucleotide frame-shift mutation $(\Delta \mathrm{G}>\mathrm{TT})$ evolved in humans. 
Based on our evidence, it is likely that non-human primates evolved strategies to limit expression of functional IFN $\lambda 4$, and consequently, neither human nor non-human primates express IFN $\lambda 4$ protein to sufficient levels for biological consequences.

\subsection{DISCUSSION}

IFNL4 is a member of the type III IFNs that was most recently identified through a genetic association study ${ }^{216}$. Genetic studies postulate a cell-autonomous, intracellular role for IFN $\lambda 4$ in dampening the antiviral response, but have failed to provide functional support for this hypothesis. Therefore, we carried out a comprehensive biochemical and molecular study to investigate the functional role of IFN $\lambda 4$ during viral infections.

Our IFN $\lambda 4$ overexpression studies show that the IFN $\lambda 4$ p179 variant can be secreted and has comparable antiviral activities to IFN $\lambda 3$, confirming previous data ${ }^{219,224}$. We extended these observations by producing recombinant full-length IFN $\lambda 4$ p179 protein in Drosophila S2 cells, further demonstrating that antiviral activity of IFN $\lambda 4$ is preserved and its potency against viruses such as $\mathrm{HCV}$ and $\mathrm{WNV}$ is comparable to that of IFN $\lambda 3$. We further observed that neither intracellular nor secreted IFN $\lambda 4$ isoforms interfered with type I or III IFN signaling, as they did not affect induction of ISGs via either type I or type III IFN receptors. These observations are paradoxical to the findings from genetic association studies in HCV patients, where the $\Delta \mathrm{G}$ allele that codes for full-length IFNLAP179 associates with a worse clinical outcome ${ }^{216,222,232}$.

We proceeded to examine whether endogenous IFN $\lambda 4$ acts similarly during PAMP stimulations or viral infection. By cloning endogenous IFNLA isoforms from stimulated hepatocytes, we found additional mRNA splice variants with intron retention in the cDNA, which would not be translated as the introns prevent their export to the cytoplasm. This discovery 
warranted a comprehensive expression profiling analysis of IFNL4 isoforms induced in multiple cell lines and primary human hepatocytes containing both variants at rs368234815 ( $\Delta \mathrm{G}$ and TT) during stimulation with PAMP and upon viral infections. Although previous studies documented induction of IFNL4 ${ }^{227}$, they have used primer/probes that do not differentiate the isoforms and in most cases amplify functional and non-functional isoforms indiscriminately. Using qPCR probes specific for each transcript, we were surprised to find that the intron-retaining and IFNLP107 isoforms were the most abundant among all IFNL4 isoforms regardless of their rs 368234815 genotype. More intriguingly, IFNL3 was induced several (2-3) logs-fold higher than any IFNL4 splice forms. This induction pattern was consistent irrespective of PAMP stimulations or viral infections. We also determined that endogenous IFNL4 isoforms are not efficiently translated into proteins compared to IFNL3 as they are poorly loaded onto polyribosomes upon PAMP stimulation. Notably, IFNL4 is the only IFNL gene that does not encode a strong canonical polyA signal, which recruits proteins that cleave and polyadenylate the primary mRNA. Polyadenylation sequences then lead to the recruitment of several RNA-binding proteins essential for mRNA stability and subsequent translation. Longer polyA tails provide higher mRNA stability and higher translation potential ${ }^{236}$. Indeed, we found poor usage of its non-canonical polyA signal. Based on these data, we hypothesize that the IFNL4 3' UTR could be playing additional roles in reducing the expression levels and lowering the translation potential of IFNLA.

Altogether, we show that multiple mechanisms combine to minimize IFN $\lambda 4$ protein expression in the host, including alternative splicing of IFNL4 for significant production of nonfunctional proteins, preferential expression of unstable intron-retaining mRNA variants, poor loading onto polyribosomes for protein production and a weak polyA signal that further lowers the stability and translation potential of all IFNL4 splice forms (Fig. 3.6). In addition to these 
regulatory controls, humans have evolved a frame-shift " $\Delta \mathrm{G}>\mathrm{TT}$ " mutation that further disrupts the coding potential of the IFNL4 gene. While non-human primates bear only the ancestral $\Delta \mathrm{G}$ allele and thus do not have this recent adaptation, we documented favored induction of nonfunctional IFNL4 isoforms compared to the functional IFNLAP179 isoform in gorilla fibroblasts, as we observed in human cells. We propose that these splicing and translational control mechanisms to suppress expression of functional IFN $\lambda 4$ protein are manifestations of more evolutionarily ancient selection against the production and function of IFN $\lambda 4$ during infection, prior to the frame shift mutation that evolved in humans to further silence its expression. Our mechanistic evidence shows that humans have sustained adaptations suppressing IFN $\lambda 4$ activity even when the genotype at rs368234815 allows production of in-frame IFN $\lambda 4$. Taken together, this study highlights differential activities of the IFNL genes during viral infection and the relatively low contribution of IFNL4 compared to IFNL3 in physiological immune contexts. A question of why bioactive IFNL4 expression is so greatly suppressed, but not IFNL3 can only be speculated. Similar to other interferon/cytokine genes, IFNL4 may have arisen from gene duplication but failed to subfunctionalize or neofunctionalize leading to high redundancy with IFNL3. It is also possible that IFNL4 may have more complex pathological roles detrimental to the host which led to its suppression. These are just speculations that can be tested only if the functional IFNL4 is expressed at physiological levels during infection. We and others have previously found the IFNL3 variant to have functional effects on antiviral immunity against HCV infection and unfortunately, high linkage disequilibrium makes it difficult to distinguish the contributing effects of individual polymorphism. It remains to be seen whether the clinical association of IFNL4 gene expression with poor HCV clearance is simply explained by the tightly 
linked genetic association of the unfavorable IFNL4 $\triangle \mathrm{G}$ genotype with the unfavorable IFNL3 genotypes and not a direct biological effect of IFNL4.

\subsection{MATERIALS AND METHODS}

Cell culture conditions. HepG2, PH5CH8, Huh7, HeLa, HEK293 cells and Gorilla gorilla fibroblasts were cultured in complete DMEM (Sigma) (cDMEM) media containing 10\% heatinactivated fetal bovine serum (FBS; Atlanta Biologicals) and 1\% penicillin-streptomycinglutamine (PSG; Mediatech). The cells were incubated at $37{ }^{\circ} \mathrm{C}$ with $5 \% \mathrm{CO}_{2}$. Primary human hepatocytes $(\mathrm{PHH})$ were purchased from Life Technologies and cultured according to the vendor's instructions. THP-1 cells were grown in complete RPMI 1640 containing 10\% FBS, PSG, 10mM HEPES, 1mM sodium pyruvate, $1 \mathrm{X}$ non-essential amino acids (Mediatech) and $50 \mu \mathrm{M} 2$ mecaptoethanol (Sigma). MUTZ-3 cells were grown in MEM $\alpha$ containing nucleotides (Gibco), $20 \%$ FBS and $10 \%$ conditioned medium from 5637 renal carcinoma cells. To differentiate myeloid cell lines into dendritic cells, THP-1 and MUTZ-3 cells were seeded at $0.2 \times 10^{6}$ cells/ml and cultured for 7 days in the presence of cytokines (for THP-1, GM-CSF 100 ng/ml and IL-4 100 ng/ml in complete RPMI 1640; for MUTZ-3, GM-CSF 100 ng/ml, IL-4 10 ng/ml and TNFa 2.5 $\mathrm{ng} / \mathrm{ml}$ in MEM $\alpha$ containing nucleotides, $20 \% \mathrm{FBS}$ ), with half the medium replaced every 3 days.

Generation of IFNLRI ${ }^{-/}$hepatocytes. IFNLRI targeting guide RNA (gRNA, 5'GCTCTCCCACCCGTAGACGG-3') was cloned downstream of the U6 promoter in the pRRLU6empty-gRNA-MND-cas9-t2A-Puro vector using In-Fusion enzyme mix (Clontech). Hepatocytes were transfected with either cas9-expressing or IFNLRI gRNA-cas9-expressing plasmids. For transfection of Huh7 cells, $3 \times 10^{6}$ cells were seeded onto a $10 \mathrm{~cm}$ dish and $10 \mu \mathrm{g}$ of plasmid was 
transfected using the $\mathrm{CaPO}_{4}$ transfection kit (Invitrogen) according to the manufacturer's instructions. After $48 \mathrm{~h}$ cells were pre-selected by addition of $2 \mu \mathrm{g} / \mathrm{ml}$ puromycin to the media for 2 days. To confirm successful gene targeting in pre-selected cells, genomic DNA was extracted from wt/cas9 control and IFNLRI ${ }^{-/-}$cells (NucleoSpin Tissue; Clontech) and subjected to T7 endonuclease I assay. Pre-selected cells were then single-cell sub-cloned and analyzed for IFNLR1 ${ }^{-}$ /- knockout efficiency by checking for downstream activation of STAT1 and MXI induction upon stimulation with IFN $\lambda 3$.

Stimulations. IFN $\beta$ was purchased from PBL and used at $100 \mathrm{IU} / \mathrm{ml}$ each. IFN $\lambda 3$ was purchased from R\&D Systems and used at $100 \mathrm{ng} / \mathrm{ml}$. Neutralizing IL-10R2 antibody (MAB874, R\&D) was pre-incubated with cells at 2-6 $\mu \mathrm{g} / \mathrm{ml}$ for one hour prior to cytokine stimulation. Poly(I:C) (InvivoGen) was used at $1 \mu \mathrm{g} / \mathrm{ml}$. The RIG-I ligand HCV 5'ppp RNA (HCV PAMP) was transcribed in vitro and used at $1 \mu \mathrm{g} / \mathrm{ml}$. Both were transfected into cell lines using XtremeGene HP (Roche) or Mirus TransIT-X2 reagent (Mirus). Primary human hepatocytes were stimulated by adding $1 \mu \mathrm{g} / \mathrm{ml}$ poly(I:C) directly to the culture media.

Cloning and sequencing of IFNL4 isoforms. We amplified IFNL4 from HepG2 cells stimulated with poly(I:C) for $12 \mathrm{~h}$ and the mRNA was amplified with IFNL4-cDNA fwd and IFNL4-cDNA rev primers (Suppl. Table 1). The amplified products were cloned into a pCR2.1 TA-cloning vector (Invitrogen) and the inserts were sequenced. IFNL4P107, IFNL4P131 and IFNL4P179 were cloned into a C-terminal HA vector [pCMV-HA(c) pHOM-Mem1]. The three isoforms were amplified using the primers IFNL4-FL-HA-fwd and IFNL4-FL-HA-rev (Table 3.1) and cloned into the vector using EcoRI and SpeI restriction sites. To identify 3' UTR lengths and sequences of 
endogenous IFNL4, 3' Rapid Amplification of cDNA Ends (3' RACE) was carried out based on the manufacturer's instruction (Invitrogen). IFNL4-RACE-nest1 and IFNL4-RACE-nest2 forward primers (Table 3.1) were used for 3' RACE to amplify the 3' UTR of IFNL4. The 3' RACE products were cloned into a pCR2.1 TA-cloning vector and sequenced as above.

RNA isolation, reverse transcription and quantification of gene expression. Total RNA was isolated using the NucleoSpin RNA kit (Macherey-Nagel) according to the manufacturer's protocol. cDNA was synthesized from $1 \mu \mathrm{g}$ total RNA using the QuantiTect RT kit (Qiagen) according to manufacturer's instructions. qPCR was carried out using the ViiA7 qPCR system with TaqMan reagents (Life Technologies) using custom-made isoform-specific IFNL4 TaqMan probes (Table 3.1, IDT), IFNL3, IFNB, ACTB, HPRT and interferon stimulated genes $M X 1$, OAS1 and ISG15 (Life Technologies). Gene expression levels were normalized to either ACTB or HPRT.

Immunoblot analysis. $30 \mu \mathrm{g}$ of cell lysates, $10 \mu \mathrm{l}$ supernatant or $1 \mathrm{ml}$ TCA precipitated supernatant from cell culture were subjected to SDS-PAGE and transferred to PVDF membranes (Thermo Scientific). The membranes were then probed in $5 \%$ BSA in TBST (Tris-Buffered Saline and Tween 20) or $5 \%$ non-fat milk in TBST for IFN $\lambda 4$ (4G1, Millipore), phospho-STAT1 (Y701) (58D6, Cell Signaling), STAT1 (42H3, Cell Signaling), HA (6E2, Cell Signaling) or $\beta$-actin (13E5, Cell Signaling).

Interferon bioactivity reporter assay. An interferon-stimulated response element (ISRE)luciferase reporter construct along with eGFP control and overexpression constructs for IFN $\lambda 3$ or IFN $\lambda 4$ isoforms were co-transfected using XtremeGene HP (Roche) into Huh7 cells plated in a 
96-well plate. 24 h post transfection, the cells were lysed with 1x Passive Lysis Buffer (Promega) and luciferase and eGFP values were measured using a multi-mode microplate reader (Synergy HT, BioTek).

Culture conditions of S2 cells. Drosophila Schneider 2 cells (S2) were grown in complete Schneider's Drosophila Medium (Gibco, Invitrogen), 10\% heat-inactivated fetal bovine serum, penicillin-streptomycin (Gibco) and $20 \mu \mathrm{g} / \mathrm{ml}$ gentamycin (Amresco).

Production of recombinant IFN $\lambda 4$ protein. We expressed IFN $\lambda 4$ in a Drosophila S2 cell expression system (Invitrogen). The cells were stably co-transfected with plasmids encoding IFNL4-Histag and a blasticidin resistance gene in a ratio of 19:1 using calcium phosphate (Invitrogen). Cells were then passed into ExpressFive serum-free medium (Invitrogen) containing $25 \mu \mathrm{g} / \mathrm{ml}$ blasticidin and scaled up under constant selection to $1 \mathrm{~L}$ suspension cultures at $125 \mathrm{rpm}$ in spinner flasks. At a density of $5.0 \times 10^{6}$ cells $/ \mathrm{ml}$, cells were induced by $0.8 \mathrm{mM} \mathrm{CuSO}_{4}$ to produce IFN $\lambda 4$ for eight days. Recombinant IFN $\lambda 4$ protein was isolated from the supernatant by affinity chromatography, eluted in an imidazole gradient on a $\mathrm{Ni} 2^{+}$-IDA-based His60 resin (Clontech). The eluate was analyzed by a Coomassie gel to identify enriched fractions, which were subsequently concentrated by ultracentrifugation columns and desalted in PBS using PD-10 columns (GE Healthcare). To remove non-specifically-bound proteins, we performed size exclusion chromatography using an ÄKTA 9 high pressure liquid chromatography system with a Superdex 200 analytical column (Amersham Biosciences). Finally, we added $0.1 \%$ BSA as a carrier protein and froze single-use aliquots in $20 \%$ glycerol. 
Cell death assays. PH5CH8 cells were treated with either IFN $\lambda 4(100 \mathrm{ng} / \mathrm{ml})$, IFN $\lambda 3$ (100 ng/ml) or actinomycin D (ActD, $10 \mu \mathrm{g} / \mathrm{ml}$ ) (Thermo Fisher). Cell death and confluence were assessed over time using an IncuCyte (Essen Bioscience) imaging system for $70 \mathrm{~h}$ with $100 \mathrm{nM}$ Sytox Green (Thermo Fisher), a cell-impermeable DNA-binding fluorescent dye which stains only dead cells.

Genotyping assays. Genotyping assays were performed using TaqMan primers and probes as previously described $^{216}$ (Table 3.1).

Virus infections. HCV-Renilla infections were carried out as previously described ${ }^{233}$. Briefly, Huh7 cells $\left(1.5 \times 10^{5}\right.$ cells $\left./ \mathrm{ml}\right)$ were plated on 96-well plates in cDMEM and incubated overnight at $37^{\circ} \mathrm{C}$ to ensure $60-70 \%$ confluency. We infected the cells with HCV-Renilla (MOI=0.3) diluted in serum-free DMEM in a total volume of $35 \mu \mathrm{l}$ for $4 \mathrm{~h}$. The media was replaced with $100 \mu \mathrm{l}$ cDMEM and the cell lysate was harvested for luciferase assay $48 \mathrm{~h}$ after infection. WNV isolate TX 2002-HC (WNV-TX) was described previously ${ }^{237}$. Dengue virus type 2 (DV2) stocks were generated from seed stocks provided by A. Hirsch and J. A. Nelson (Oregon Health and Sciences University). Virus stocks were titered with a standard plaque assay on Vero cells. Huh7 cells were infected at MOI=1-2 with either WNV-TX or DV2, or $25 \mathrm{HAU} / \mathrm{ml} \mathrm{SeV} \mathrm{Cantell} \mathrm{strain} \mathrm{(Charles}$ River) diluted in serum-free DMEM, or mock infected. The virus inoculum was removed $2 \mathrm{~h}$ post infection and replaced with cDMEM supplemented with $10 \%$ FBS. Total RNA was extracted using the NucleoSpin RNA kit, treated with DNase I (Ambion) and evaluated by real-time qPCR for relative gene expression and intracellular viral RNA levels using SYBR Green (Applied Biosystems). Real-time qPCR methods for quantifying intracellular WNV viral RNA is described previously ${ }^{238}$. 
Polysome analyses. $10 \times 10^{6} \mathrm{HepG} 2($ IFNL4 $\triangle \mathrm{G} / \mathrm{TT})$ cells were stimulated with poly(I:C) for $6 \mathrm{~h}$. Cells were then treated with $100 \mu \mathrm{g} / \mathrm{ml}$ of cycloheximide (MP Biomedicals) for $5 \mathrm{~min}$, then washed twice with ice-cold PBS and harvested. The cell pellet was resuspended in polysome lysis buffer and cells were left to lyse on ice for $20 \mathrm{~min}$, then centrifuged at $8,000 \mathrm{x} \mathrm{g}$ for $10 \mathrm{~min}$ at $4{ }^{\circ} \mathrm{C}$. Supernatants were layered above $10 \%-50 \%$ sucrose gradient and centrifuged at 36,000 rpm for 2 h $30 \mathrm{~min}$ at $4{ }^{\circ} \mathrm{C}$. Gradients were fractionated while continuously monitoring absorbance at 254 nm.

Statistical analyses. Statistics were performed using one-way ANOVA in GraphPad Prism 6.0. For Figure 1L, multiple comparisons were made against EV-transfected cells; for Figure 3A-B, multiple comparisons were made against mock-treated cells. 


\subsection{FIGURES}

A

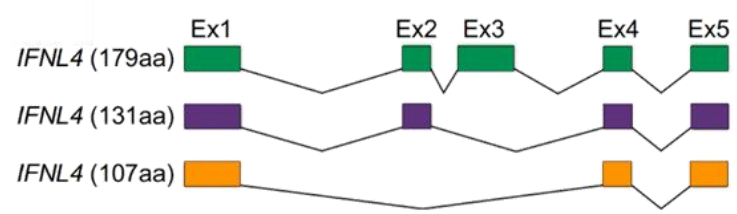

B

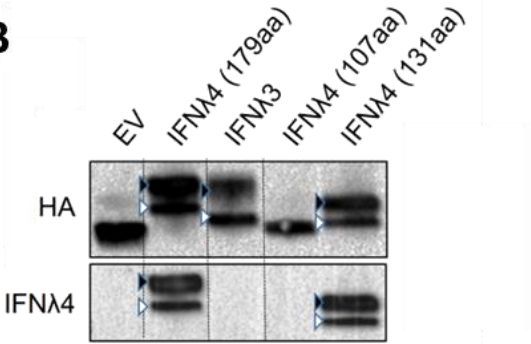

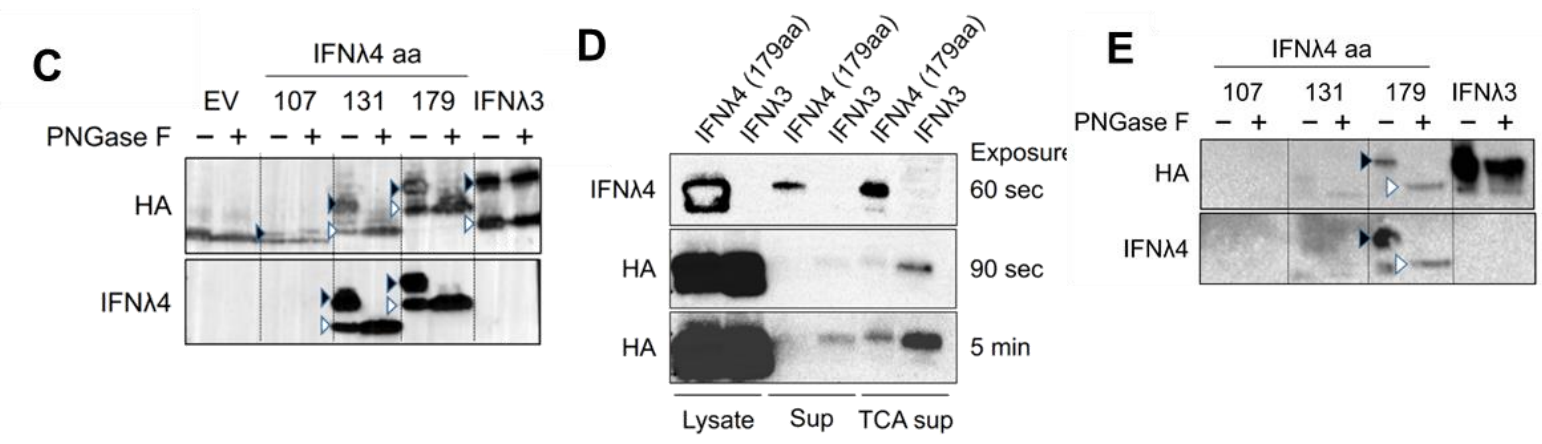
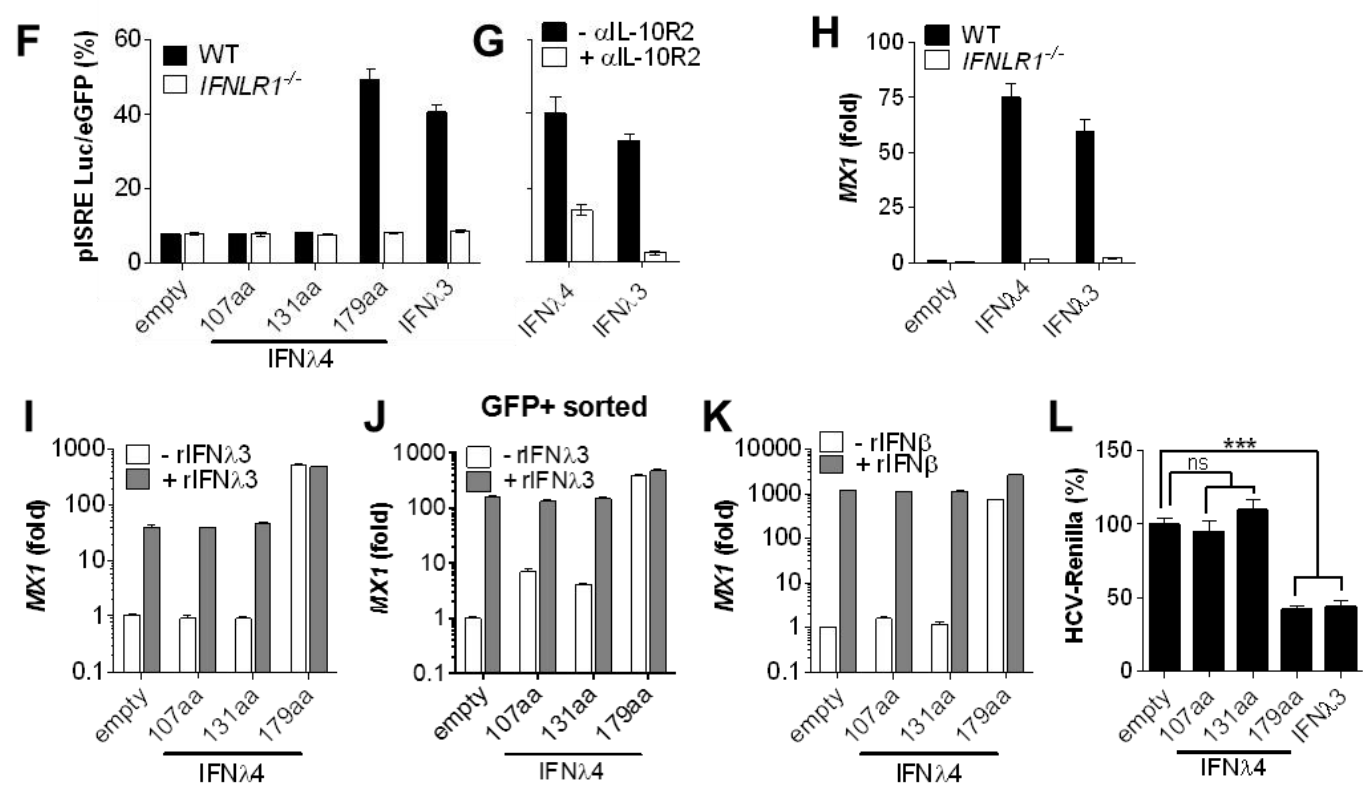

Figure 3.1. IFN $\lambda 4 p 179$ and IFN $\lambda 3$ have similar biological activities.

(A) Schematic of the gene structure of protein-coding splice variants of IFNL4 generated by alternative splicing. (B) Immunoblot of HA-tagged IFN $\lambda 3$ and IFN $\lambda 4$ isoforms overexpressed in Huh7 cells. (C) Immunoblot of PNGase F-treated lysates from cells overexpressing HA-IFN $\lambda 4$ isoforms or HA-IFN $\lambda 3$. (D) Immunoblot of cell lysates, neat supernatants and supernatants concentrated by trichloroacetic acid (TCA) treatment. (E) Immunoblot of TCA-treated supernatants of Huh7 cells overexpressing IFN $\lambda 4$ isoforms. (F) Luciferase reporter assay measuring ISRE activity in wild-type and IFNLRI ${ }^{-/}$Huh7 cells overexpressing IFN $\lambda 3$ or IFN $\lambda 4$ 
isoforms. (G) Luciferase reporter assay measuring ISRE activity in Huh7 cells treated with IL10R2 neutralizing antibody and overexpressing IFN $\lambda 4 p 179$ and IFN $\lambda 3$. (H) $M X 1$ expression in wild-type and IFNLR $1^{-/}$Huh7 cells overexpressing IFN $\lambda 4 p 179$ and IFN $\lambda 3$. (I-K) $M X 1$ expression in Huh7 cells overexpressing IFN $\lambda 4$ isoforms and treated with either IFN $\lambda 3$ (I) or IFN $\beta$ (K). (J) MX1 expression after co-transfection of a GFP plasmid along with the IFNL4 and IFNL3 overexpression constructs sorting of $\mathrm{GFP}^{+}$cells before IFN $\lambda 3$ stimulation. (L) Renilla luciferase reporter activity measured in Huh7 cells overexpressing IFN $\lambda 3$ or IFN $\lambda 4$ isoforms infected with with hepatitis $\mathrm{C}$ virus (HCV) tagged with Renilla luciferase. Above experiments are representative of at least 2-3 biological replicates. (B, C\&E) Solid arrows: glycosylated forms; clear arrows: deglycosylated forms. $* * * \mathrm{p}<0.001$, $\mathrm{ns}=$ not significant. 

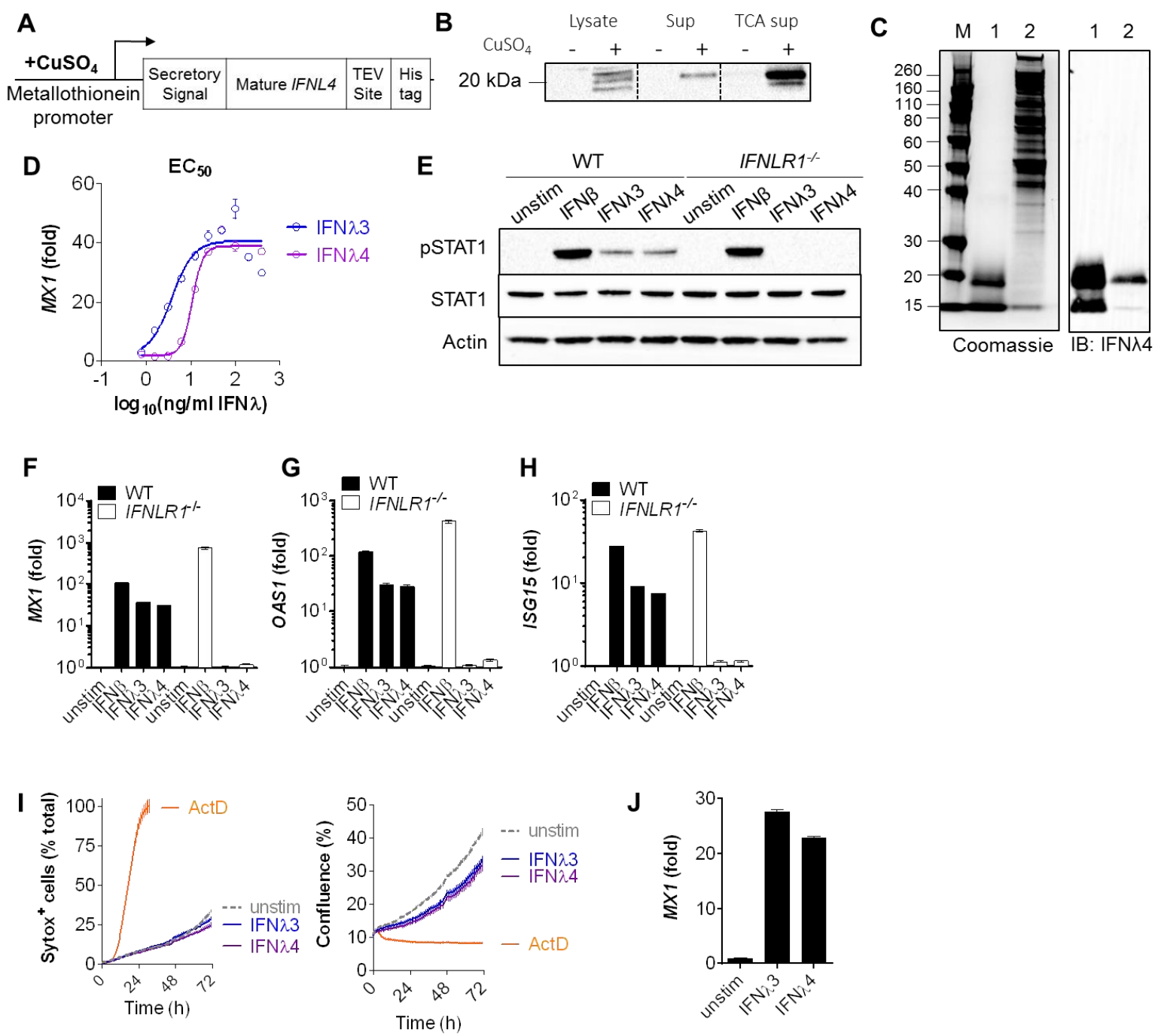

Figure 3.2. Recombinant IFN $\lambda 4$ induces ISGs but not cell death.

(A) Schematic of the plasmid of the inducible expression of recombinant human IFN $\lambda 4 \mathrm{p} 179$ (rhIFN入4) in a Drosophila S2 Schneider cell expression system. (B) Immunoblot showing expression of rhIFN $\lambda 4$ in lysates and supernatants upon treatment with copper (II) sulfate for 8 days. (C) Coomassie stain and immunoblot of rhIFN $\lambda 4$ purified by affinity chromatography and gel filtration. Lane: $\mathrm{M}$, molecular weight marker; 1, purified rhIFN $\lambda 4$; 2, pre-purified supernatant from $\mathrm{S} 2$ cells. (D) $\mathrm{EC}_{50}$ curve of IFN $\lambda 3$ and IFN $\lambda 4$ activity assayed by $M X 1$ gene expression. (E) Immunoblot of pSTAT1 in wild-type and $I F N L R 1^{-/-} \mathrm{PH} 5 \mathrm{CH} 8$ cells, treated with recombinant IFN $\beta$, IFN $\lambda 3$ and IFN $\lambda 4$. (F-H) Gene expression of MXI (F), OASI (G) and ISG15 (H) in wildtype and IFNLRI ${ }^{-1}$ PH5CH8 cells treated with recombinant IFN $\beta$, IFN $\lambda 3$ or IFN $\lambda 4$ for 6 h. (I) Proliferation and cell death (object counts) in wild-type PH5CH8 hepatocytes stimulated with 
IFN $\lambda 3$, IFN $\lambda 4$ and actinomycin D (positive control), measured using Incucyte time-lapse live fluorescent microscopy of a quantifiable cell viability dye Sytox green, which marks dead cells. (J) $M X 1$ gene expression measured at $70 \mathrm{~h}$ to confirm stimulation of the hepatocytes by IFN $\lambda 3$ and IFN $\lambda 4$ in cell death assays. Above experiments are representative of at least 2-3 biological replicates. 
A

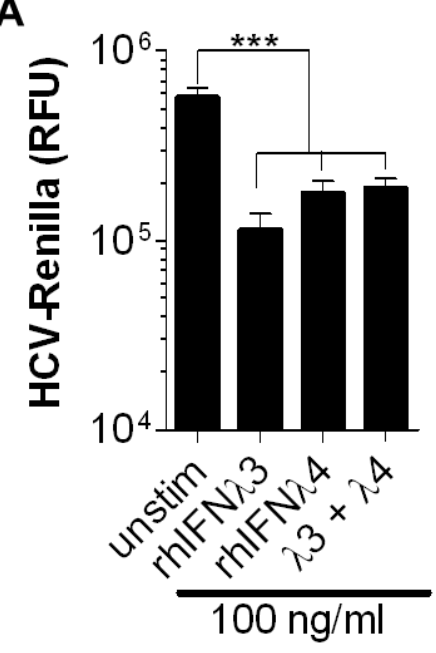

B

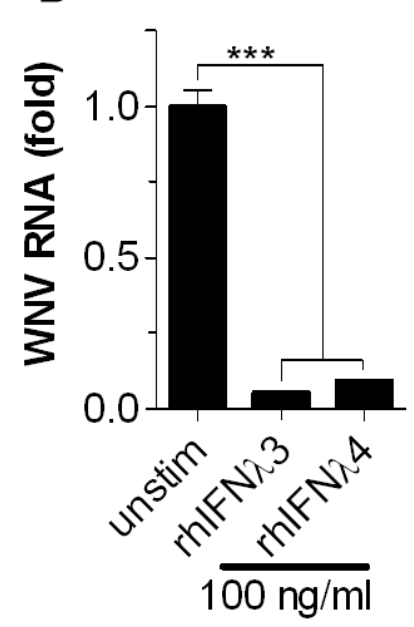

C

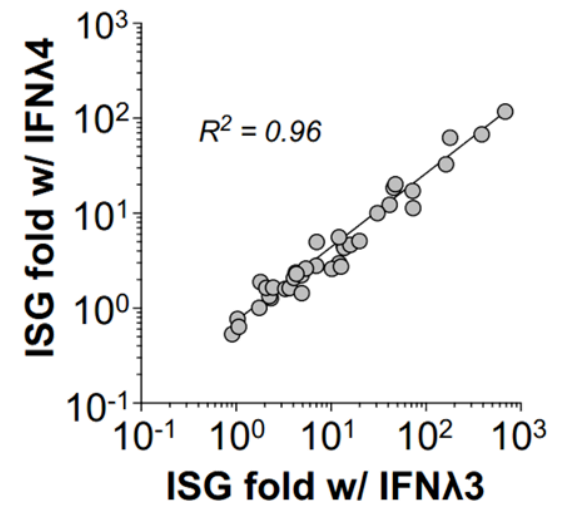

Figure 3.3. Recombinant human IFN $\lambda 4$ shows similar antiviral activity to IFN $\lambda 3$.

(A) Renilla luciferase reporter activity measuring HCV replication in Huh7 cells infected with Renilla luciferase-tagged HCV and treated with rhIFN $\lambda 3$ and rhIFN $\lambda 4$. (B) West Nile virus (WNV) RNA load upon treatment of infected Huh7 cells with equal doses of rhIFN $\lambda 3$ and rhIFN $\lambda 4$. (C) Correlation of ISG induction in WNV-infected Huh7 cells (from Fig. 3B) stimulated with 100 $\mathrm{ng} / \mathrm{ml} \mathrm{rhIFN} \lambda 3$ and rhIFN $\lambda 4$, based on a TaqMan qPCR array of 37 ISGs. Above experiments are representative of at least 2-3 biological replicates. ${ }^{* * *} \mathrm{p}<0.001$. 


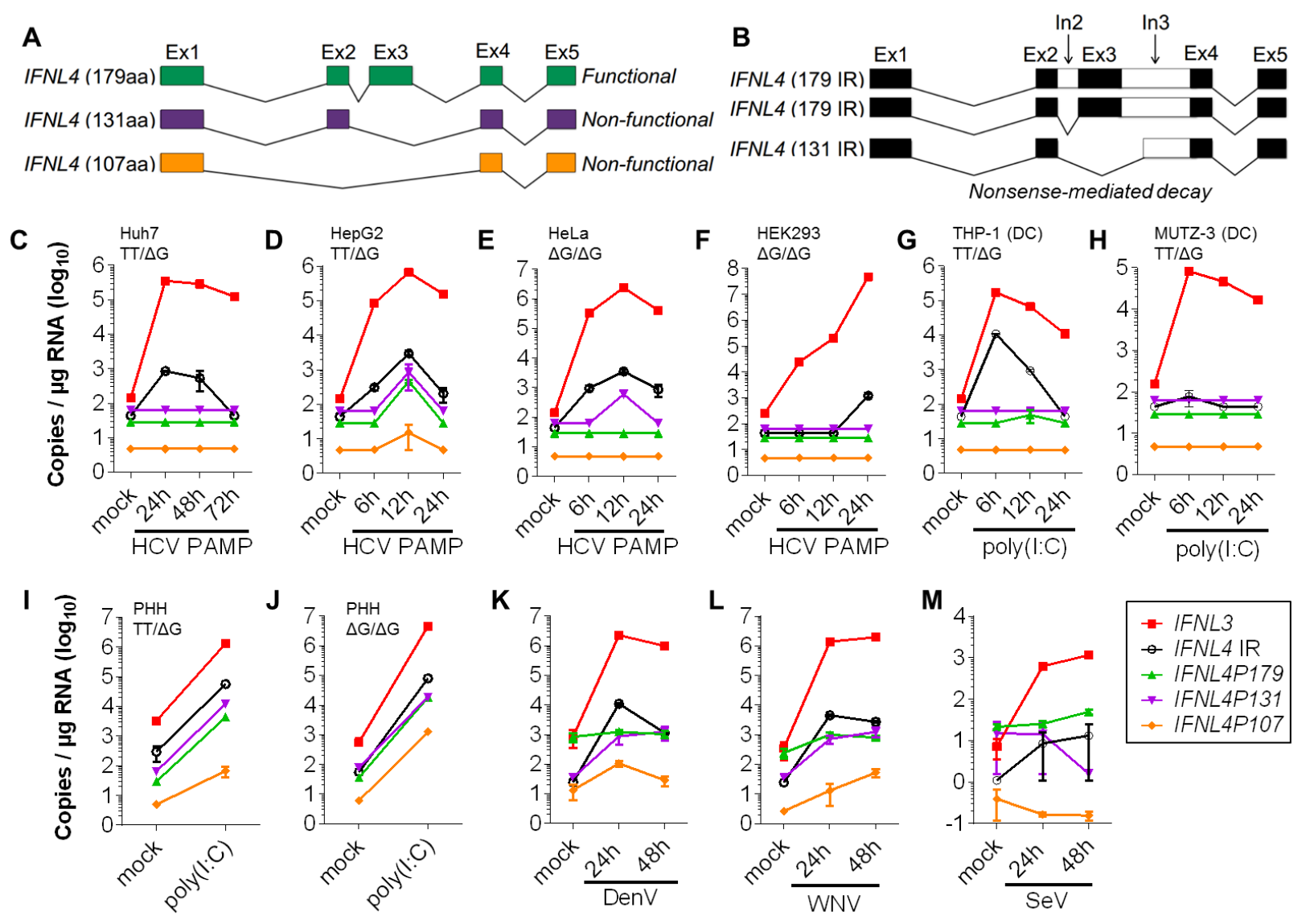

Figure 3.4. Low IFNL4 induction during PAMP stimulation and viral infection.

(A-B) Schematic of IFNL4 mRNA exhibiting intron retention (IR), compared to the known protein-coding isoforms of IFNL4. (C-H) Gene expression kinetics of IFNL3 and IFNL4 isoforms measured by real-time PCR using custom made TaqMan qPCR probes in Huh7 (C), HepG2 (D), HeLa (E), HEK293 (F) cells upon stimulation with HCV PAMP, and DCs derived from THP1 (G) and MUTZ-3 (H) cell lines stimulated with poly(I:C). (I-J) Gene expression of IFNL3 and IFNLA isoforms in primary human hepatocytes, heterozygous (I) or homozygous (J) for the $\Delta \mathrm{G}$ variant, stimulated with poly(I:C). (K-M) Gene expression of IFNL3 and IFNL4 isoforms in HepG2 cells infected with Dengue virus (K), West Nile virus (L) and Sendai virus (M). All experiments are representative of at least 2-3 biological replicates. 
A
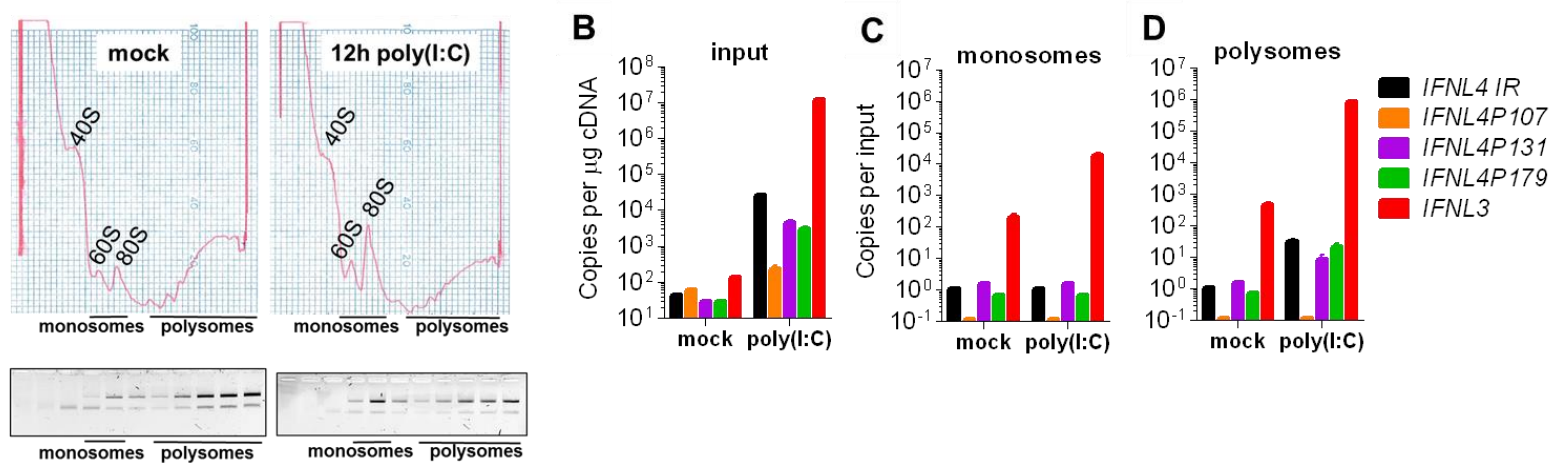

E

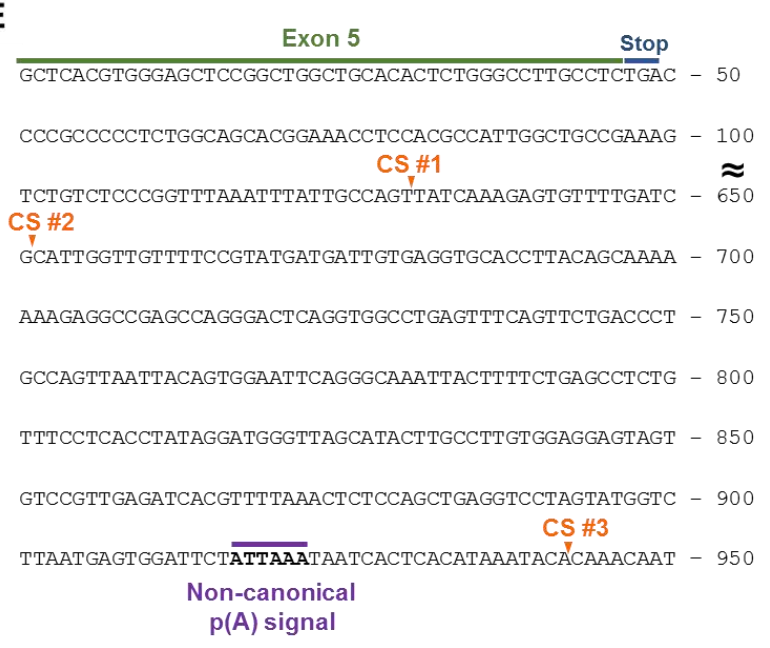

F

$\begin{gathered}\text { Cleavage site usage } \\
\text { (no. of clones) }\end{gathered}$
\begin{tabular}{|c|c|}
\hline CS \#1 & $1 / 17(5.9 \%)$ \\
\hline CS \#2 & $12 / 17(70.6 \%)$ \\
\hline CS \#3 & $4 / 17(23.5 \%)$ \\
\hline
\end{tabular}

Figure 3.5. IFNL4 mRNA are poorly loaded onto polyribosomes for translation and suppressed in evolution.

(A) Whole-cell extracts from mock or poly(I:C) treated HepG2 were resolved by density sedimentation in 10-50\% sucrose gradients. The UV absorbance trace (254 nm) obtained during fractionation is shown with the positions of the $40 \mathrm{~S}, 60 \mathrm{~S}, 80 \mathrm{~S}$, and polyribosomes. The lower panel shows an agarose gel of polysome fractions to check for $28 \mathrm{~S}$ and $18 \mathrm{~S}$ ribosomal RNA in the fractions. Copy number expression of IFNL3 and IFNL4 isoforms in input (B), monosome (C), and polysome (D) fractions measured by qPCR. (E) Cleavage sites (CS) and frequency of CS usage in the human IFNL4 3' UTR determined by 3' RACE. (F) Gene expression of IFNL3 and IFNL4 isoforms in gorilla fibroblasts upon stimulation with poly(I:C). All experiments are representative of at least 2-3 biological replicates. 


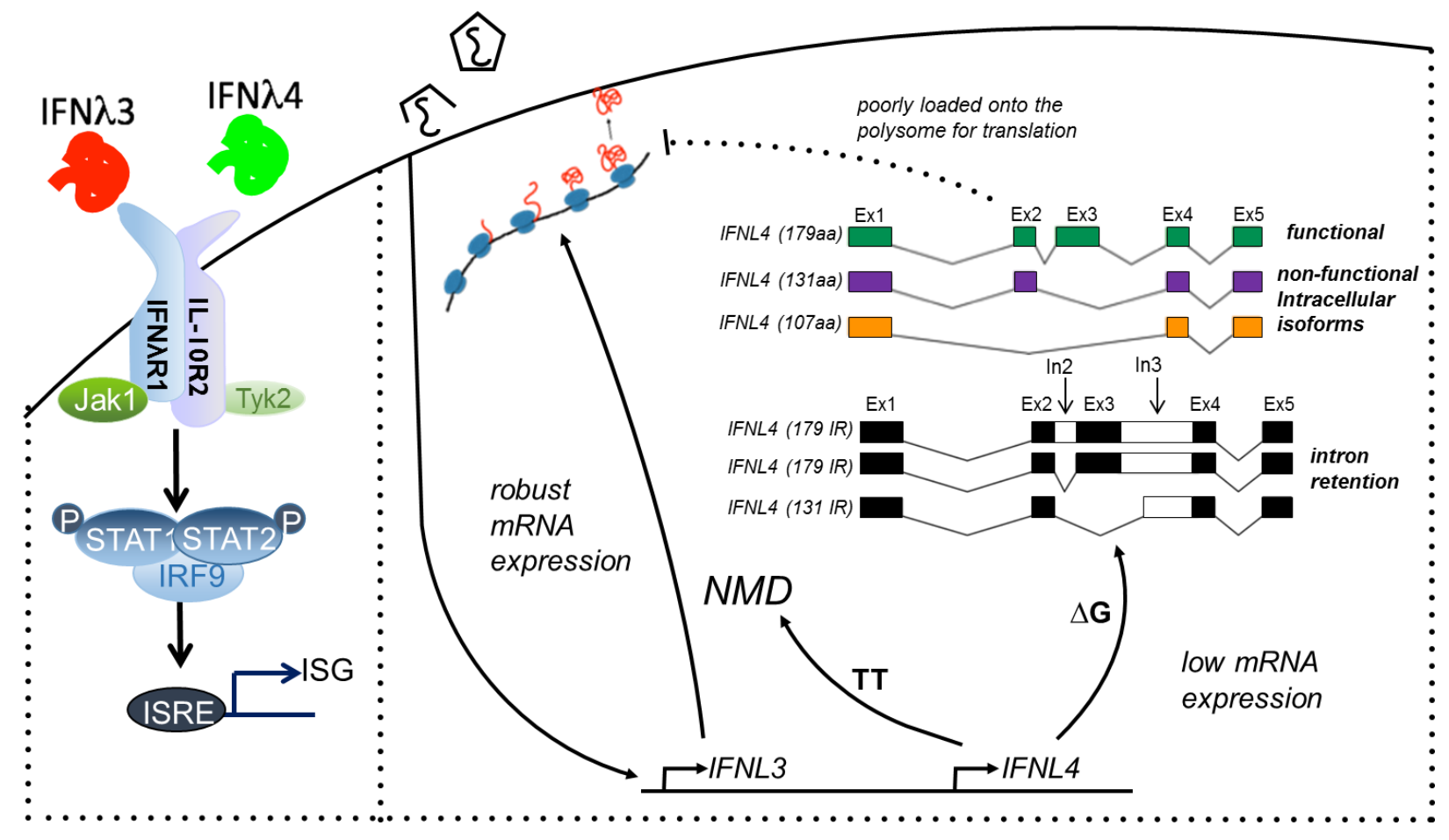

Figure 3.6. IFN $\lambda 4$ expression is suppressed during viral infections.

IFN $\lambda 4$ p179 has similar antiviral properties as IFN $\lambda 3$, signaling exclusively through the extracellular IFN $\lambda$ R1 and IL-10R2 heterodimer to activate STAT1 and induce ISGs. During viral infections, several mechanisms are in place which suppress the expression of the functional IFNLA isoform compared to robust IFNL3 expression. 

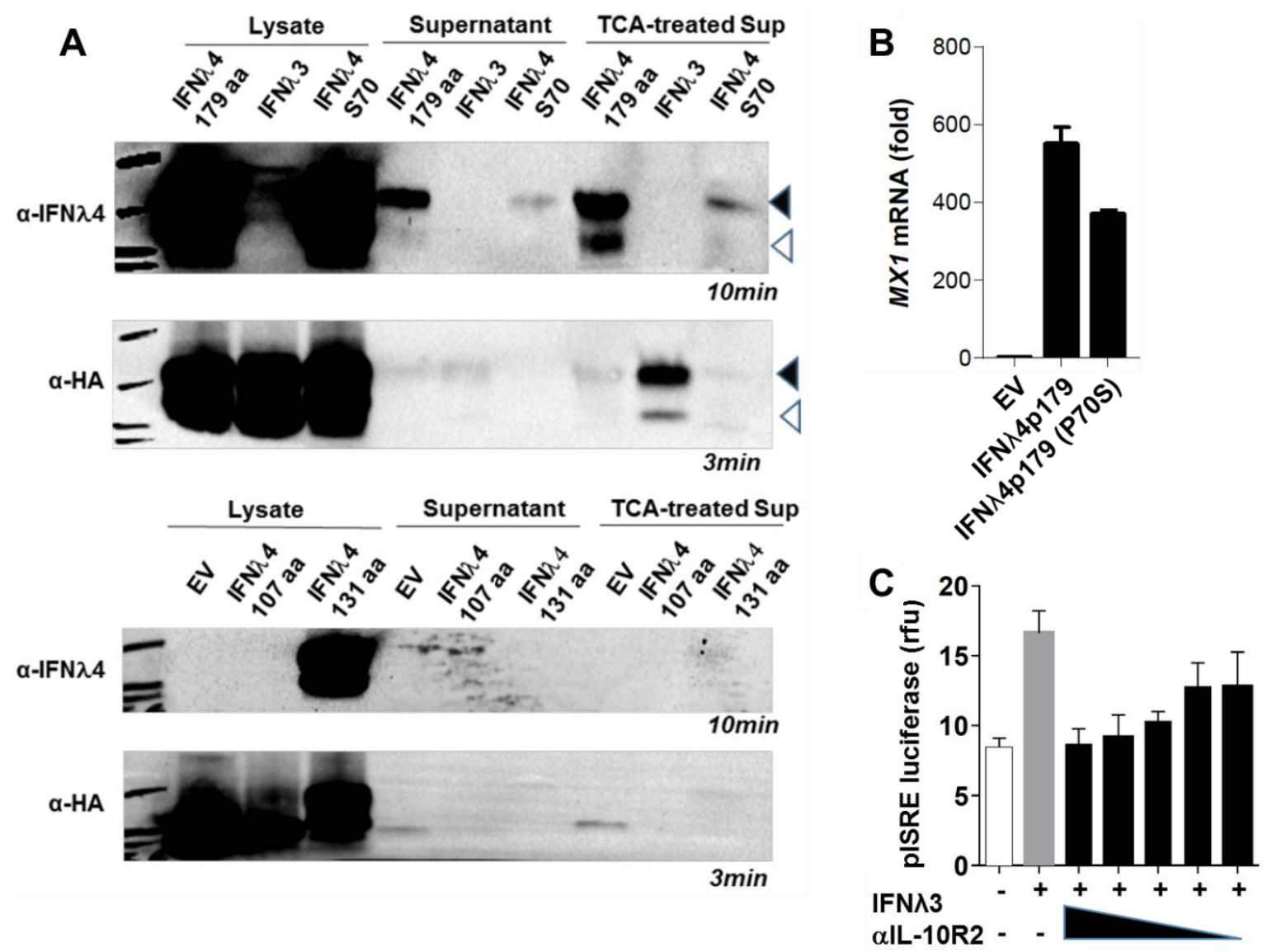

Supplementary Figure 3.1. Titration of IL-10R2 antibody.

(A) Immunoblot of lysates, neat supernatants and supernatants concentrated by trichloroacetic acid (TCA) of HA-tagged IFN $\lambda 4 p 179$ (P70), IFN $\lambda 4$ p179 P70S variant, IFN $\lambda 4 p 131$, IFN $\lambda 4 p 107$, and IFN $\lambda 3$ overexpressed in Huh7 hepatocytes. Arrows indicate glycosylated (solid) and nonglycosylated (clear) forms, respectively. (B) $M X 1$ induction upon overexpression of IFN $\lambda 4 p 179$ P70 and IFN $\lambda 4$ p179 P70S variants in Huh7 cells. (C) pISRE luciferase reporter activity in transfected Huh7 hepatocytes stimulated with rhIFN $\lambda 3$ in the presence of varying doses of $\alpha$ IL10R2 antibody $(2-6 \mu \mathrm{g} / \mathrm{ml})$. 
A

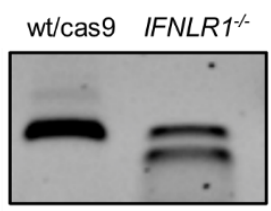

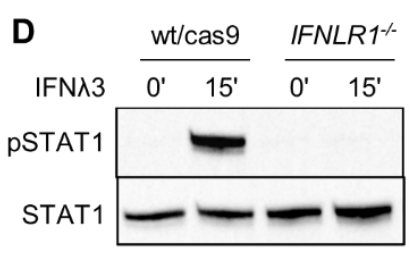

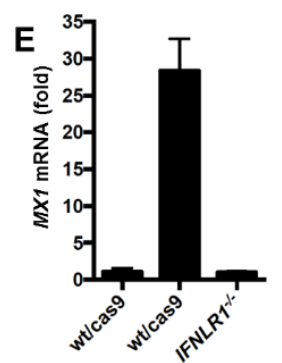

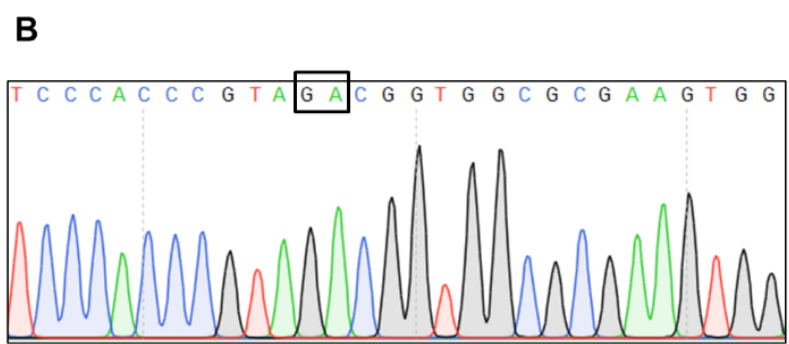

C
IFNLR1 WT

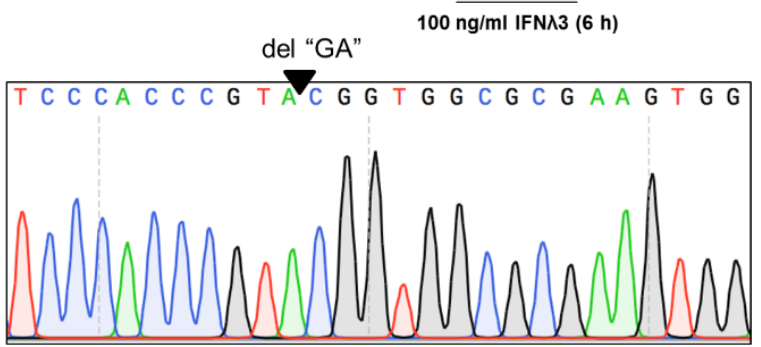

IFNLR1 KO

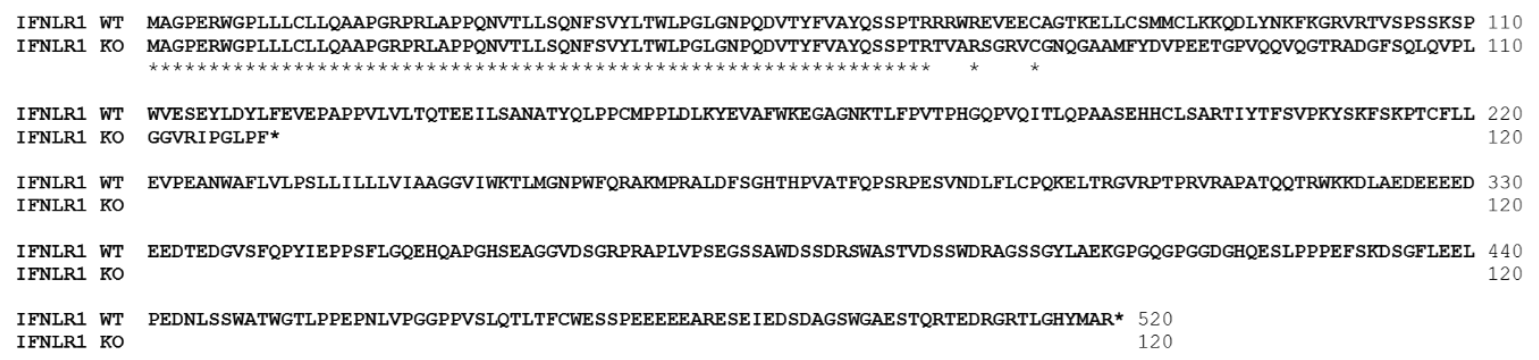

IFNLR1 WT MAGPERWGPLLLCLLQAAPGRPRLAPPQNVTLLSONFSVYLTWLPGLGNPQDVTYFVAYQSSPTRRRWREVEECAGTKELLCSMMCLKKQDLYNKFKGRVRTVSPSSKSP 110 IFNLR1 KO MAGPERWGPLLLCLLQAAPGRPRLAPPQNVTLLSQNFSVYLTWLPGLGNPQDVTYFVAYQSSPTRTVARSGRVCGNQGAAMFYDVPEETGPVQQVQGTRADGFSQLQVPL 110 *********************************************************************

IFNLR1 WT WVESEYLDYLFEVEPAPPVLVLTQTEEILSANATYQLPPCMPPLDLKYEVAFWKEGAGNKTLFPVTPHGQPVQITLQPAASEHHCLSARTIYTFSVPKYSKFSKPTCFLI 220 IFNLR1 KO GGVRIPGLPF*

IFNLR1 WT EVPEANWAFLVLPSLLILLLVIAAGGVIWKTLMGNPWFQRAKMPRALDFSGHTHPVATFQPSRPESVNDLFLCPQKELTRGVRPTPRVRAPATQQTRWKKDLAEDEEEED 330 IFNLR1 KO

IFNLR1 WT EEDTEDGVSFQPYIEPPSFLGQEHQAPGHSEAGGVDSGRPAPLVPSEGSSAWDSSDRSWASTVDSSWDRAGSSGYLAEKGPGQGPGGDGHQESLPPPEFSKDSGFLEEL 440 IFNLR1 KO

IFNLR1 WT PEDNLSSWATWGTLPPEPNLVPGGPPVSLQTLTFCWESSPEEEEEARESE IEDSDAGSWGAESTQRTEDRGRTLGHYMAR 520 IFNLR1 KO

Supplementary Figure 3.2. Generation and characterization of $I F N L R I^{-/-}$hepatocytes. (A) CRISPR/cas9-mediated IFNLRI targeting confirmed by T7 endonuclease I cleavage assay; two bands in the IFNLR1 $1^{-/}$lane indicates targeting of genomic DNA by CRISPR. (B) Sequencing chromatogram of the CRISPR target region in the human IFNLRI gene. Clonal cell line derived from CRISPR targeted IFNLRI ${ }^{-1}$ cells have a two base pair deletion $(\triangle \mathrm{GA})$ compared to wild type non-targeted cells. (C) Alignment of wild type IFNLRI amino-acid sequence with truncated CRISPR targeted IFNLRI ${ }^{-}$sequence due to a two base pair deletion resulting in a pre-mature stop codon. (D) Immunoblot of pSTAT1, total STAT1 and (E) $M X 1$ gene induction in wild type and IFNLRI ${ }^{-}$PH5CH8 cells stimulated with rhIFN $\lambda 3$ for $6 \mathrm{~h}$. 
Exon 1

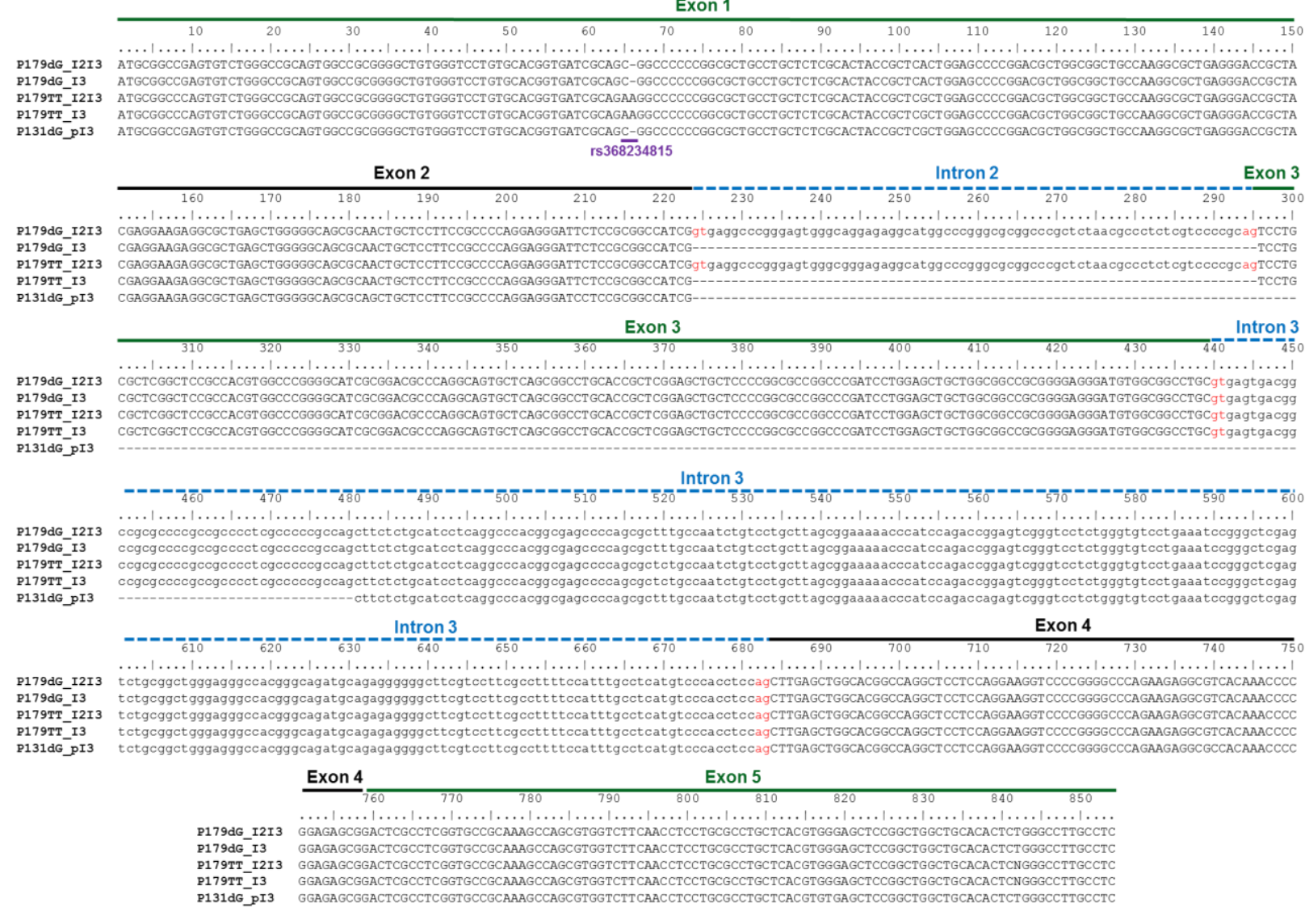

\section{Supplementary Figure 3.3. Sequence alignment of intron-retaining IFNL4 isoforms identified from a cDNA library from HepG2 cells treated with poly(I:C).}

Alternating exons are labelled with solid lines and capital letters, introns with dotted lines and small letters, position of rs368234815 in purple, and intronic splice donor and acceptor sites in red. Multiple sequence alignment was performed using CLUSTALW in BioEdit sequence alignment editor (http://www.mbio.ncsu.edu/bioedit/bioedit.html). 


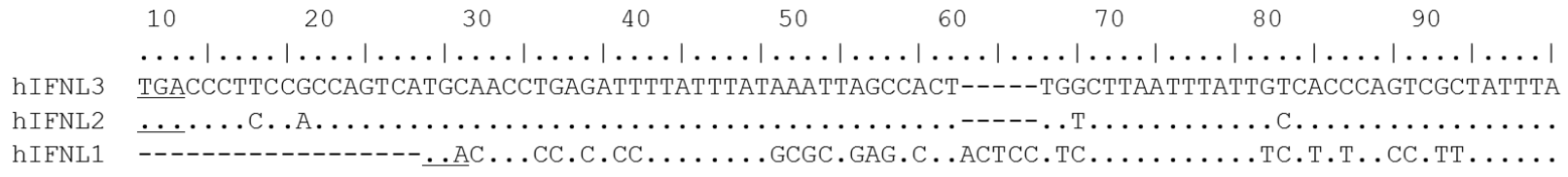

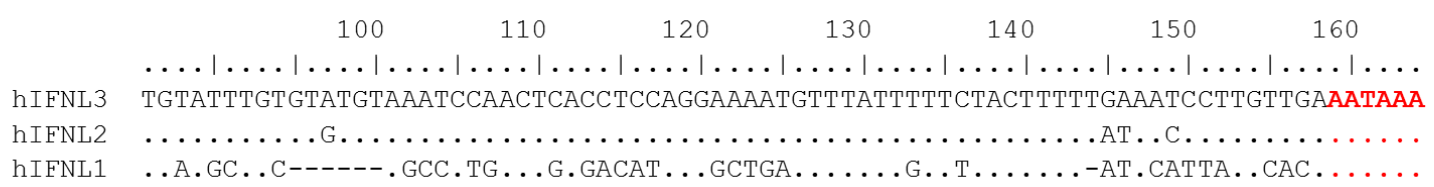

Supplementary Figure 3.4. Alignment of 3' UTR sequences of human IFNL1, IFNL2 and $I F N L 3$ genes.

The canonical polyadenylation signal (AATAAA) is labeled in red. Dots in IFNL2 and IFNL1 sequences indicate sequence homology to IFNL3. The stop codon (TGA) is underlined. Multiple sequence alignment was performed using CLUSTALW in BioEdit sequence alignment editor (http://www.mbio.ncsu.edu/bioedit/bioedit.html). 


\subsection{TABLES}

Table 3.1. Sequences of primers and probes used to clone, detect and genotype IFNL4.

\begin{tabular}{|c|c|}
\hline Primer/probe name & Sequence $\left(5^{\prime}>3^{\prime}\right)$ \\
\hline IFNL4-cDNA fwd & ATGCGGCCGAGTGTCTGG \\
\hline IFNL4-cDNA rev & GAGGCAAGGCCCAGAGTGT \\
\hline IFNL4-FL-HA-fwd & GAATTCGCGCGTATGCGGCCGAGTGTCTGG \\
\hline IFNL4-FL-HA-rev & ACTAGTGAGGCAAGGCCCAGAGTGT \\
\hline IFNL4-RACE-nest1 & GTGGTCTTCAACCTCCTGCG \\
\hline IFNL4-RACE-nest2 & CTGCTCACGTGGGAGCTCCG \\
\hline IFNL4-Genot-fwd & ATGCGGCCGAGTGTCTGG \\
\hline IFNL4-Genot-rev & GCTCCAGCGAGCGGTAGTG \\
\hline IFNL4- $\Delta$ G probe & GGCCGCTGCGAT \\
\hline IFNL4-TT probe & GGCCTTCTGCGAT \\
\hline IFNL4P179-fwd & CGATCCTGGAGCTGCTG \\
\hline IFNL4P179-rev & TTTGTGACGCCTCTTCTGG \\
\hline IFNL4P179 probe & CAAGGCAGGCCGCCACATC \\
\hline IFNL4P131-fwd & CAACTGCTCCTTCCGCC \\
\hline IFNL4P131-rev & GGGTTTGTGACGCCTCTTC \\
\hline IFNL4P131 probe & CGGCCATCGCTTGAGCTGG \\
\hline IFNL4P107-fwd & CTCTCGCACTACCGCTC \\
\hline IFNL4P107-rev & TTTGTGACGCCTCTTCTGG \\
\hline IFNL4P107 probe & CGCTGAGGGACCGCTACCTTG \\
\hline IFNL4 IR-fwd & GTCCTTCGCCTTTTCCATTTG \\
\hline IFNL4 IR-rev & AGACCACGCTGGCTTTG \\
\hline IFNL4 IR probe & CGTCACAAACCCCGGAGAGCG \\
\hline
\end{tabular}




\section{Chapter 4. CONCLUDING REMARKS}

Alternative splicing is a powerful tool used by eukaryotes to increase diversity in their proteome from a finite genome. Exon skipping, use of alternative splice sites and different transcription start sites allows the use of different exon combinations to generate proteins of containing different permutations of functional domains, allowing them to fine-tune their biological activities. In addition, intron retention nonsense-mediated decay

We have investigated how the isoforms of IL-22BP and IFN $\lambda 4$ differ in their functions and examined their differential expression. Both are generated by splice variation of their parent genes in patterns seemingly specific to humans and higher primates. While these cytokine families appear unrelated at first glance, activating different STAT pathways and occupying quite different physiological niches, the combined results from both studies add fascinating diversity to how protein isoforms change immune function. These are important aspects of cytokine function that escape detection in genetic association studies, and are also poorly modelled in animals due to high species specificity of splicing decisions. In approaching these questions for both genes, we first examined their biological activities using overexpression systems and purification of glycosylated recombinant protein from an eukaryotic expression system. With the knowledge of their differential activities, we could then examine the relative expression of each isoform in different cells or in response to different stimuli to put into perspective where each specific function comes into play in immunity.

Alternative splicing of human IL-22BP results in three variants of the soluble receptor that perform different functions in IL-22 signaling. The inclusion of exon 3 in IL-22BPi1 renders it inactive, while the inclusion or exclusion of exon 5 and most of exon 6 generates two variants of the protein with two different levels of antagonism, IL-22BPi2 and IL-22BPi3. This splicing 
flexibility allows it to fine-tune IL-22 signaling in a gradient generated between homeostatic and inflammatory conditions.

The hand of the spliceosome is heavy in changing the activity of IL-22BP. IL-22BPi3 is generated by skipping of exon 5 and an alternative splice site in exon 6 that encodes a frame shift and truncates the protein. This results in the absence of the second fibronectin domain of IL22BPi2 that is known to contain one important stabilizing interaction with IL-22. While this alone causes major functional changes to the antagonistic activity of IL-22BP, the wildly different expression patterns of IL-22BPi2 and IL-22BPi3 in homeostasis and inflammation make it clear that tissue-specific and myeloid-specific factors further alter the biological relevance of IL-22BPi2 and IL-22BPi3 by transforming splice site usage. Meanwhile, the inactivation of IL-22BPi1 by exonization of a retrotransposon recalls previous observations in IL-15, which uses alternative transcription start sites and alternative exon 4 usage results to generate different signal peptides that control the intracellular trafficking, secretion and consequent activity of the cytokine. In both these cases, alternative splicing enables expression of a transcript, yet limit its bioactivity; at least for IL-22BPi1, this mechanism emerged in higher primates and appears to have been evolutionarily selected for since. Since the dose and location of IL-22 signaling dictates its highly context-dependent roles, perhaps separating the parent genes of the membrane-bound and soluble receptors allows more flexible diversification of isoforms that have provided greater dynamic range and sensitivity for tweaking IL-22 signaling.

In contrast, we found that the generation of alternative splice forms of IFN $\lambda 4$ serves mostly as a mechanism to limit expression of a functional cytokine. In addition to three known proteincoding isoforms, we identified a high abundance of transcripts generated with retained introns, which would target the IFNL4 mRNA for NMD and prevent expression of a productive cytokine. 
Yet these transcripts are expressed in preference to the protein-coding isoforms, and among these only one is truly secreted from the cell and active in signaling. As discussed in section 1.1.3, splicing of transcripts to achieve NMD is being increasingly recognized as a legitimate way to efficiently suppress the expression of an active, functional protein, so this mechanism is not without precedent. For IFN $\lambda 4$, splicing is exploited to suppress a transcript whose expression is genetically associated with detrimental effects in infection. The truly unique properties of IFN $\lambda 4$ and its isoforms among the IFN families have present a fresh perspective on how it is regulated differently from other members, as type I IFNs are single-exon transcripts that cannot undergo alternative splicing, while alternative transcripts have not been found for type II and III IFNs. Along with the paradoxical pro-viral association, for which an explanation still has not been satisfactorily presented, splicing as a regulatory mechanism adds to IFN $\lambda 4$ 's status as the oddball of the IFN family.

Our ability to obtain these insights required a combination approach in both studies: first to determine the biological function of overexpressed or purified proteins and then to examine their endogenous expression. These aspects were often examined separately in previous IL-22BP and IFN $\lambda 4$ studies, which made it difficult to determine overall where and how they would act. Being able to clarify both how IL-22BPi3 functioned and where it was expressed in comparison to IL-22BPi2, for example, allowed us to propose the rheostat model of regulation, whereas such a consideration could not be confidently made with either finding in isolation. Similarly, while the discovery of intron retention revealed a new, important regulatory mechanism for IFN $\lambda 4$, verifying the biological inactivity of the additional protein-coding isoforms IFN $\lambda 4 p 107$ and IFN $\lambda 4 p 131$ against hypotheses in the field for non-canonical functions of these proteins provided a larger context that even "productive" transcripts contributed to suppression of IFN $\lambda 4$ activity. Therefore, 
both remain important questions that should be approached simultaneously when studying novel regulators of cytokine signaling.

Overall, our studies on the human IL-22BP and IFN $\lambda 4$ isoforms have united studies of function and regulation to demonstrate the central role alternative splicing has to controlling signaling in cytokine responses. The mechanisms we have discovered for the regulation of IL22BP and IFN $\lambda 4$ function have precedent in other genes and transcriptomic networks, and it is with delight that we add mucosal immunity and antiviral responses among their number. Furthermore, our findings on the function of alternative splicing in these two gene families are exciting additions to the lush regulatory landscape of the immune system, a network that especially requires tight control of timing, dose and strength of its responses. 


\section{REFERENCES}

1 Pan, Q., Shai, O., Lee, L. J., Frey, B. J. \& Blencowe, B. J. Deep surveying of alternative splicing complexity in the human transcriptome by high-throughput sequencing. Nat Genet 40, 1413-1415, doi:10.1038/ng.259 (2008).

2 Kornblihtt, A. R. et al. Alternative splicing: a pivotal step between eukaryotic transcription and translation. Nature reviews. Molecular cell biology 14, 153-165, doi:10.1038/nrm3525 (2013).

3 Wang, G. S. \& Cooper, T. A. Splicing in disease: disruption of the splicing code and the decoding machinery. Nature reviews. Genetics 8, 749-761, doi:10.1038/nrg2164 (2007).

4 Braunschweig, U., Gueroussov, S., Plocik, A. M., Graveley, B. R. \& Blencowe, B. J. Dynamic integration of splicing within gene regulatory pathways. Cell 152, 1252-1269, doi:10.1016/j.cell.2013.02.034 (2013).

5 Pagani, F. et al. New type of disease causing mutations: the example of the composite exonic regulatory elements of splicing in CFTR exon 12. Human molecular genetics 12, 1111-1120 (2003).

6 Pagani, F. et al. A new type of mutation causes a splicing defect in ATM. Nat Genet 30, 426-429, doi:10.1038/ng858 (2002).

7 Steiner, B., Truninger, K., Sanz, J., Schaller, A. \& Gallati, S. The role of common singlenucleotide polymorphisms on exon 9 and exon 12 skipping in nonmutated CFTR alleles. Human mutation 24, 120-129, doi:10.1002/humu.20064 (2004).

8 Pagani, F., Raponi, M. \& Baralle, F. E. Synonymous mutations in CFTR exon 12 affect splicing and are not neutral in evolution. Proc Natl Acad Sci U S A 102, 6368-6372, doi:10.1073/pnas.0502288102 (2005).

9 Hassan, M. A., Butty, V., Jensen, K. D. \& Saeij, J. P. The genetic basis for individual differences in mRNA splicing and APOBEC1 editing activity in murine macrophages. Genome research 24, 377-389, doi:10.1101/gr.166033.113 (2014).

10 Kim, J. et al. Transcriptome landscape of the human placenta. BMC genomics 13, 115, doi:10.1186/1471-2164-13-115 (2012).

11 Sahebi, M. et al. Towards understanding pre-mRNA splicing mechanisms and the role of SR proteins. Gene 587, 107-119, doi:10.1016/j.gene.2016.04.057 (2016).

12 Barbosa-Morais, N. L. et al. The evolutionary landscape of alternative splicing in vertebrate species. Science 338, 1587-1593, doi:10.1126/science.1230612 (2012).

13 Calarco, J. A. et al. Global analysis of alternative splicing differences between humans and chimpanzees. Genes Dev 21, 2963-2975, doi:10.1101/gad.1606907 (2007).

14 Garlanda, C., Dinarello, C. A. \& Mantovani, A. The interleukin-1 family: back to the future. Immunity 39, 1003-1018, doi:10.1016/j.immuni.2013.11.010 (2013).

15 Burns, K. et al. Inhibition of interleukin 1 receptor/Toll-like receptor signaling through the alternatively spliced, short form of MyD88 is due to its failure to recruit IRAK-4. $J$ Exp Med 197, 263-268 (2003).

16 Janssens, S., Burns, K., Tschopp, J. \& Beyaert, R. Regulation of interleukin-1- and lipopolysaccharide-induced NF-kappaB activation by alternative splicing of MyD88. Current biology : CB 12, 467-471 (2002). 
17 Janssens, S., Burns, K., Vercammen, E., Tschopp, J. \& Beyaert, R. MyD88S, a splice variant of MyD88, differentially modulates NF-kappaB- and AP-1-dependent gene expression. FEBS letters 548, 103-107 (2003).

18 Jensen, L. E. \& Whitehead, A. S. IRAK1b, a novel alternative splice variant of interleukin1 receptor-associated kinase (IRAK), mediates interleukin-1 signaling and has prolonged stability. J Biol Chem 276, 29037-29044, doi:10.1074/jbc.M103815200 (2001).

19 Rao, N., Nguyen, S., Ngo, K. \& Fung-Leung, W. P. A novel splice variant of interleukin1 receptor (IL-1R)-associated kinase 1 plays a negative regulatory role in Toll/IL-1Rinduced inflammatory signaling. Molecular and cellular biology 25, 6521-6532, doi:10.1128/MCB.25.15.6521-6532.2005 (2005).

20 Lewis, B. P., Green, R. E. \& Brenner, S. E. Evidence for the widespread coupling of alternative splicing and nonsense-mediated mRNA decay in humans. Proc Natl Acad Sci U S A 100, 189-192, doi:10.1073/pnas.0136770100 (2003).

21 Popp, M. W. \& Maquat, L. E. Organizing principles of mammalian nonsense-mediated mRNA decay. Annual review of genetics 47, 139-165, doi:10.1146/annurev-genet-111212133424 (2013).

22 Hamid, F. M. \& Makeyev, E. V. Emerging functions of alternative splicing coupled with nonsense-mediated decay. Biochemical Society transactions 42, 1168-1173, doi:10.1042/BST20140066 (2014).

23 Barash, Y. et al. Deciphering the splicing code. Nature 465, 53-59, doi:10.1038/nature09000 (2010).

24 Lopez-Bigas, N., Audit, B., Ouzounis, C., Parra, G. \& Guigo, R. Are splicing mutations the most frequent cause of hereditary disease? FEBS letters 579, 1900-1903, doi:10.1016/j.febslet.2005.02.047 (2005).

25 Tazi, J., Bakkour, N. \& Stamm, S. Alternative splicing and disease. Biochimica et biophysica acta 1792, 14-26, doi:10.1016/j.bbadis.2008.09.017 (2009).

26 Diederichs, S. et al. The dark matter of the cancer genome: aberrations in regulatory elements, untranslated regions, splice sites, non-coding RNA and synonymous mutations. EMBO molecular medicine 8, 442-457, doi:10.15252/emmm.201506055 (2016).

27 Carpenter, S., Ricci, E. P., Mercier, B. C., Moore, M. J. \& Fitzgerald, K. A. Posttranscriptional regulation of gene expression in innate immunity. Nature reviews. Immunology 14, 361-376, doi:10.1038/nri3682 (2014).

28 Belicha-Villanueva, A. et al. What is the role of alternate splicing in antigen presentation by major histocompatibility complex class I molecules? Immunologic research 46, 32-44, doi:10.1007/s12026-009-8123-8 (2010).

29 Sahoo, A. \& Im, S. H. Interleukin and interleukin receptor diversity: role of alternative splicing. International reviews of immunology 29, 77-109, doi:10.3109/08830180903349651 (2010).

30 Schaper, F. \& Rose-John, S. Interleukin-6: Biology, signaling and strategies of blockade. Cytokine \& growth factor reviews 26, 475-487, doi:10.1016/j.cytogfr.2015.07.004 (2015).

31 Bihl, M. P. et al. Identification of a novel IL-6 isoform binding to the endogenous IL-6 receptor. American journal of respiratory cell and molecular biology 27, 48-56, doi:10.1165/ajrcmb.27.1.4637 (2002).

32 Thabard, W. et al. Myeloma cells release soluble interleukin-6Ralpha in relation to disease progression by two distinct mechanisms: alternative splicing and proteolytic cleavage. 
Clinical cancer research : an official journal of the American Association for Cancer Research 5, 2693-2697 (1999).

33 Lamas, J. R. et al. Alternative splicing and proteolytic rupture contribute to the generation of soluble IL-6 receptors (sIL-6R) in rheumatoid arthritis. Cytokine 61, 720-723, doi:10.1016/j.cyto.2012.12.025 (2013).

34 Diamant, M. et al. Cloning and expression of an alternatively spliced mRNA encoding a soluble form of the human interleukin-6 signal transducer gp130. FEBS letters 412, 379384 (1997).

35 Wolf, J. et al. Different soluble forms of the interleukin-6 family signal transducer gp130 fine-tune the blockade of interleukin-6 trans-signaling. $J$ Biol Chem, doi:10.1074/jbc.M116.718551 (2016).

36 Scheller, J., Garbers, C. \& Rose-John, S. Interleukin-6: from basic biology to selective blockade of pro-inflammatory activities. Seminars in immunology 26, 2-12, doi:10.1016/j.smim.2013.11.002 (2014).

37 Campbell, I. L. et al. Trans-signaling is a dominant mechanism for the pathogenic actions of interleukin-6 in the brain. The Journal of neuroscience : the official journal of the Society for Neuroscience 34, 2503-2513, doi:10.1523/JNEUROSCI.2830-13.2014 (2014).

38 Nowell, M. A. et al. Therapeutic targeting of IL-6 trans signaling counteracts STAT3 control of experimental inflammatory arthritis. J Immunol 182, 613-622 (2009).

39 Chalaris, A., Garbers, C., Rabe, B., Rose-John, S. \& Scheller, J. The soluble Interleukin 6 receptor: generation and role in inflammation and cancer. European journal of cell biology 90, 484-494, doi:10.1016/j.ejcb.2010.10.007 (2011).

40 Wolf, J. et al. A soluble form of the interleukin-6 family signal transducer gp130 is dimerized via a C-terminal disulfide bridge resulting from alternative mRNA splicing. Biochemical and biophysical research communications 470, 870-876, doi:10.1016/j.bbrc.2016.01.127 (2016).

41 Sommer, J. et al. Alternative intronic polyadenylation generates the interleukin-6 transsignaling inhibitor sgp130-E10. J Biol Chem 289, 22140-22150, doi:10.1074/jbc.M114.560938 (2014).

42 Stonier, S. W. \& Schluns, K. S. Trans-presentation: a novel mechanism regulating IL-15 delivery and responses. Immunology letters 127, 85-92, doi:10.1016/j.imlet.2009.09.009 (2010).

43 Onu, A., Pohl, T., Krause, H. \& Bulfone-Paus, S. Regulation of IL-15 secretion via the leader peptide of two IL-15 isoforms. J Immunol 158, 255-262 (1997).

44 Gaggero, A. et al. Differential intracellular trafficking, secretion and endosomal localization of two IL-15 isoforms. European journal of immunology 29, 1265-1274, doi:10.1002/(SICI)1521-4141(199904)29:04\&\#60;1265::AID-

IMMU1265\&\#62;3.0.CO;2-V (1999).

45 Bamford, R. N., DeFilippis, A. P., Azimi, N., Kurys, G. \& Waldmann, T. A. The 5' untranslated region, signal peptide, and the coding sequence of the carboxyl terminus of IL-15 participate in its multifaceted translational control. J Immunol 160, 4418-4426 (1998).

46 Kurys, G., Tagaya, Y., Bamford, R., Hanover, J. A. \& Waldmann, T. A. The long signal peptide isoform and its alternative processing direct the intracellular trafficking of interleukin-15. J Biol Chem 275, 30653-30659, doi:10.1074/jbc.M002373200 (2000). 
47 Bergamaschi, C. et al. Secretion and biological activity of short signal peptide IL-15 is chaperoned by IL-15 receptor alpha in vivo. $J$ Immunol 183, 3064-3072, doi:10.4049/jimmunol.0900693 (2009).

48 Nishimura, H., Washizu, J., Nakamura, N., Enomoto, A. \& Yoshikai, Y. Translational efficiency is up-regulated by alternative exon in murine IL-15 mRNA. J Immunol 160, 936-942 (1998).

49 Zhao, L. et al. An activation-induced IL-15 isoform is a natural antagonist for IL-15 function. Sci Rep 6, 25822, doi:10.1038/srep25822 (2016).

50 Lee, T. L. et al. An alternatively spliced IL-15 isoform modulates abrasion-induced keratinocyte activation. The Journal of investigative dermatology 135, 1329-1337, doi:10.1038/jid.2015.17 (2015).

51 Diniz, S. N. et al. Tissue-specific expression of IL-15RA alternative splicing transcripts and its regulation by DNA methylation. European cytokine network 21, 308-318, doi:10.1684/ecn.2010.0218 (2010).

52 Bergamaschi, C. et al. Intracellular interaction of interleukin-15 with its receptor alpha during production leads to mutual stabilization and increased bioactivity. J Biol Chem 283, 4189-4199, doi:10.1074/jbc.M705725200 (2008).

53 Dubois, S., Mariner, J., Waldmann, T. A. \& Tagaya, Y. IL-15Ralpha recycles and presents IL-15 In trans to neighboring cells. Immunity 17, 537-547 (2002).

54 Muller, J. R., Waldmann, T. A., Kruhlak, M. J. \& Dubois, S. Paracrine and transpresentation functions of IL-15 are mediated by diverse splice versions of IL15Ralpha in human monocytes and dendritic cells. J Biol Chem 287, 40328-40338, doi:10.1074/jbc.M112.378612 (2012).

55 Dubois, S. et al. Natural splicing of exon 2 of human interleukin-15 receptor alpha-chain mRNA results in a shortened form with a distinct pattern of expression. J Biol Chem 274, 26978-26984 (1999).

56 Mortier, E. et al. Soluble interleukin-15 receptor alpha (IL-15R alpha)-sushi as a selective and potent agonist of IL-15 action through IL-15R beta/gamma. Hyperagonist IL-15 x IL15R alpha fusion proteins. J Biol Chem 281, 1612-1619, doi:10.1074/jbc.M508624200 (2006).

57 Bergamaschi, C. et al. Circulating IL-15 exists as heterodimeric complex with soluble IL15Ralpha in human and mouse serum. Blood 120, e1-8, doi:10.1182/blood-2011-10384362 (2012).

58 Chertova, E. et al. Characterization and favorable in vivo properties of heterodimeric soluble IL-15.IL-15Ralpha cytokine compared to IL-15 monomer. J Biol Chem 288, 18093-18103, doi:10.1074/jbc.M113.461756 (2013).

59 Rubinstein, M. P. et al. Converting IL-15 to a superagonist by binding to soluble IL15R\{alpha\}. Proc Natl Acad Sci U S A 103, 9166-9171, doi:10.1073/pnas.0600240103 (2006).

60 Hardy, M. P. et al. The soluble murine type I interferon receptor Ifnar-2 is present in serum, is independently regulated, and has both agonistic and antagonistic properties. Blood 97, 473-482 (2001).

61 Samarajiwa, S. A. et al. Soluble IFN receptor potentiates in vivo type I IFN signaling and exacerbates TLR4-mediated septic shock. $J$ Immunol 192, 4425-4435, doi:10.4049/jimmunol.1302388 (2014). 
62 Owczarek, C. M. et al. Cloning and characterization of soluble and transmembrane isoforms of a novel component of the murine type I interferon receptor, IFNAR 2. J Biol Chem 272, 23865-23870 (1997).

63 Kim, S. H., Cohen, B., Novick, D. \& Rubinstein, M. Mammalian type I interferon receptors consists of two subunits: IFNaR1 and IFNaR2. Gene 196, 279-286 (1997).

64 Abramovich, C., Ratovitski, E., Lundgren, E. \& Revel, M. Identification of mRNAs encoding two different soluble forms of the human interferon alpha-receptor. FEBS letters 338, 295-300 (1994).

65 Witte, K. et al. Despite IFN-lambda receptor expression, blood immune cells, but not keratinocytes or melanocytes, have an impaired response to type III interferons: implications for therapeutic applications of these cytokines. Genes and immunity 10, 702714, doi:10.1038/gene.2009.72 (2009).

66 Lauber, C. et al. Transcriptome analysis reveals a classical interferon signature induced by IFNlambda4 in human primary cells. Genes and immunity 16, 414-421, doi:10.1038/gene.2015.23 (2015).

67 Lynch, K. W. Consequences of regulated pre-mRNA splicing in the immune system. Nature reviews. Immunology 4, 931-940, doi:10.1038/nri1497 (2004).

68 Sanos, S. L. et al. RORgammat and commensal microflora are required for the differentiation of mucosal interleukin 22-producing NKp46+ cells. Nature immunology 10, 83-91, doi:10.1038/ni.1684 (2009).

69 Takatori, H. et al. Lymphoid tissue inducer-like cells are an innate source of IL-17 and IL22. J Exp Med 206, 35-41, doi:Doi 10.1084/Jem.20072713 (2009).

70 Goto, M. et al. Murine NKT cells produce Th17 cytokine interleukin-22. Cell Immunol 254, 81-84, doi:DOI 10.1016/j.cellimm.2008.10.002 (2009).

71 Wolk, K. et al. IL-22 increases the innate immunity of tissues. Immunity 21, 241-254, doi:10.1016/j.immuni.2004.07.007 (2004).

72 Wolk, K. et al. IL-22 regulates the expression of genes responsible for antimicrobial defense, cellular differentiation, and mobility in keratinocytes: a potential role in psoriasis. European journal of immunology 36, 1309-1323, doi:10.1002/eji.200535503 (2006).

73 Liang, S. C. et al. Interleukin (IL)-22 and IL-17 are coexpressed by Th17 cells and cooperatively enhance expression of antimicrobial peptides. J Exp Med 203, 2271-2279, doi:10.1084/jem.20061308 (2006).

74 Brand, S. et al. IL-22 is increased in active Crohn's disease and promotes proinflammatory gene expression and intestinal epithelial cell migration. American journal of physiology. Gastrointestinal and liver physiology 290, G827-838, doi:10.1152/ajpgi.00513.2005 (2006).

75 Radaeva, S., Sun, R., Pan, H. N., Hong, F. \& Gao, B. Interleukin 22 (IL-22) plays a protective role in T cell-mediated murine hepatitis: IL-22 is a survival factor for hepatocytes via STAT3 activation. Hepatology 39, 1332-1342, doi:10.1002/hep.20184 (2004).

76 Boniface, K. et al. IL-22 inhibits epidermal differentiation and induces proinflammatory gene expression and migration of human keratinocytes. J Immunol 174, 3695-3702 (2005).

77 Nagalakshmi, M. L., Rascle, A., Zurawski, S., Menon, S. \& de Waal Malefyt, R. Interleukin-22 activates STAT3 and induces IL-10 by colon epithelial cells. International immunopharmacology 4, 679-691, doi:10.1016/j.intimp.2004.01.008 (2004). 
78 Sugimoto, K. et al. IL-22 ameliorates intestinal inflammation in a mouse model of ulcerative colitis. The Journal of clinical investigation 118, 534-544, doi:10.1172/JCI33194 (2008).

79 Lamarthee, B. et al. Donor interleukin-22 and host type I interferon signaling pathway participate in intestinal graft-versus-host disease via STAT1 activation and CXCL10. Mucosal immunology 9, 309-321, doi:10.1038/mi.2015.61 (2016).

80 Hernandez, P. P. et al. Interferon-lambda and interleukin 22 act synergistically for the induction of interferon-stimulated genes and control of rotavirus infection. Nature immunology 16, 698-707, doi:10.1038/ni.3180 (2015).

81 Bachmann, M., Ulziibat, S., Hardle, L., Pfeilschifter, J. \& Muhl, H. IFNalpha converts IL22 into a cytokine efficiently activating STAT1 and its downstream targets. Biochemical pharmacology 85, 396-403, doi:10.1016/j.bcp.2012.11.004 (2013).

82 Pan, H., Hong, F., Radaeva, S. \& Gao, B. Hydrodynamic gene delivery of interleukin-22 protects the mouse liver from concanavalin A-, carbon tetrachloride-, and Fas ligandinduced injury via activation of STAT3. Cellular \& molecular immunology 1, 43-49 (2004).

83 Zheng, Y. et al. Interleukin-22, a $\mathrm{T}(\mathrm{H}) 17$ cytokine, mediates IL-23-induced dermal inflammation and acanthosis. Nature 445, 648-651, doi:10.1038/nature05505 (2007).

84 Zheng, Y. et al. Interleukin-22 mediates early host defense against attaching and effacing bacterial pathogens. Nature medicine 14, 282-289, doi:10.1038/nm1720 (2008).

85 Aujla, S. J. et al. IL-22 mediates mucosal host defense against Gram-negative bacterial pneumonia. Nature medicine 14, 275-281, doi:10.1038/nm1710 (2008).

$86 \mathrm{Ki}, \mathrm{S}$. H. et al. Interleukin-22 treatment ameliorates alcoholic liver injury in a murine model of chronic-binge ethanol feeding: role of signal transducer and activator of transcription 3. Hepatology 52, 1291-1300, doi:10.1002/hep.23837 (2010).

87 Pociask, D. A. et al. IL-22 is essential for lung epithelial repair following influenza infection. The American journal of pathology 182, 1286-1296, doi:10.1016/j.ajpath.2012.12.007 (2013).

$88 \mathrm{Xu}, \mathrm{X}$. et al. Conventional NK cells can produce IL-22 and promote host defense in Klebsiella pneumoniae pneumonia. J Immunol 192, 1778-1786, doi:10.4049/jimmunol.1300039 (2014).

89 Goto, Y. et al. Innate lymphoid cells regulate intestinal epithelial cell glycosylation. Science 345, 1254009, doi:10.1126/science.1254009 (2014).

90 Wang, X. et al. Interleukin-22 alleviates metabolic disorders and restores mucosal immunity in diabetes. Nature 514, 237-241, doi:10.1038/nature13564 (2014).

91 Pickard, J. M. et al. Rapid fucosylation of intestinal epithelium sustains host-commensal symbiosis in sickness. Nature, doi:10.1038/nature13823 (2014).

92 Park, O. et al. Biologically active, high levels of interleukin-22 inhibit hepatic gluconeogenesis but do not affect obesity and its metabolic consequences. Cell \& bioscience 5, 25, doi:10.1186/s13578-015-0015-0 (2015).

93 Shih, V. F. et al. Homeostatic IL-23 receptor signaling limits Th17 response through IL22-mediated containment of commensal microbiota. Proc Natl Acad Sci U S A 111, 1394213947, doi:10.1073/pnas.1323852111 (2014).

94 Lim, C. \& Savan, R. The role of the IL-22/IL-22R1 axis in cancer. Cytokine \& growth factor reviews 25, 257-271, doi:10.1016/j.cytogfr.2014.04.005 (2014). 
95 Martin, J. C. et al. IL-22BP is produced by eosinophils in human gut and blocks IL-22 protective actions during colitis. Mucosal immunology, doi:10.1038/mi.2015.83 (2015).

96 Huber, S. et al. IL-22BP is regulated by the inflammasome and modulates tumorigenesis in the intestine. Nature 491, 259-263, doi:10.1038/nature11535 (2012).

97 Laaksonen, H. et al. The multiple sclerosis risk gene IL22RA2 contributes to a more severe murine autoimmune neuroinflammation. Genes and immunity 15, 457-465, doi:10.1038/gene.2014.36 (2014).

98 Dumoutier, L., Lejeune, D., Colau, D. \& Renauld, J. C. Cloning and characterization of IL-22 binding protein, a natural antagonist of IL-10-related T cell-derived inducible factor/IL-22. J Immunol 166, 7090-7095 (2001).

99 de Moura, P. R. et al. Crystal structure of a soluble decoy receptor IL-22BP bound to interleukin-22. FEBS letters 583, 1072-1077, doi:10.1016/j.febslet.2009.03.006 (2009).

100 Dumoutier, L., Van Roost, E., Ameye, G., Michaux, L. \& Renauld, J. C. IL-TIF/IL-22: genomic organization and mapping of the human and mouse genes. Genes and immunity 1, 488-494, doi:10.1038/sj.gene.6363716 (2000).

101 Igawa, D., Sakai, M. \& Savan, R. An unexpected discovery of two interferon gamma-like genes along with interleukin (IL)-22 and -26 from teleost: IL-22 and -26 genes have been described for the first time outside mammals. Molecular immunology 43, 999-1009, doi:10.1016/j.molimm.2005.05.009 (2006).

102 Duhen, T., Geiger, R., Jarrossay, D., Lanzavecchia, A. \& Sallusto, F. Production of interleukin 22 but not interleukin 17 by a subset of human skin-homing memory T cells. Nature immunology 10, 857-863, doi:10.1038/ni.1767 (2009).

103 Eyerich, S. et al. Th22 cells represent a distinct human T cell subset involved in epidermal immunity and remodeling. The Journal of clinical investigation 119, 3573-3585, doi:10.1172/JCI40202 (2009).

104 Trifari, S., Kaplan, C. D., Tran, E. H., Crellin, N. K. \& Spits, H. Identification of a human helper $\mathrm{T}$ cell population that has abundant production of interleukin 22 and is distinct from $\mathrm{T}(\mathrm{H})-17, \mathrm{~T}(\mathrm{H}) 1$ and $\mathrm{T}(\mathrm{H}) 2$ cells. Nature immunology 10, 864-871, doi:10.1038/ni.1770 (2009).

105 Mielke, L. A. et al. Retinoic acid expression associates with enhanced IL-22 production by gammadelta $\mathrm{T}$ cells and innate lymphoid cells and attenuation of intestinal inflammation. J Exp Med 210, 1117-1124, doi:10.1084/jem.20121588 (2013).

106 Cella, M. et al. A human natural killer cell subset provides an innate source of IL-22 for mucosal immunity. Nature 457, 722-725, doi:10.1038/nature07537 (2009).

107 Kirchberger, S. et al. Innate lymphoid cells sustain colon cancer through production of interleukin-22 in a mouse model. $J$ Exp Med 210, 917-931, doi:10.1084/jem.20122308 (2013).

108 Rutz, S., Eidenschenk, C. \& Ouyang, W. IL-22, not simply a Th17 cytokine. Immunological reviews 252, 116-132, doi:10.1111/imr.12027 (2013).

109 Sabat, R., Ouyang, W. \& Wolk, K. Therapeutic opportunities of the IL-22-IL-22R1 system. Nature reviews. Drug discovery 13, 21-38, doi:10.1038/nrd4176 (2013).

110 Mizoguchi, A. Healing of intestinal inflammation by IL-22. Inflammatory bowel diseases 18, 1777-1784, doi:10.1002/ibd.22929 (2012).

111 Aggarwal, S., Xie, M. H., Maruoka, M., Foster, J. \& Gurney, A. L. Acinar cells of the pancreas are a target of interleukin-22. Journal of interferon \& cytokine research : the 
official journal of the International Society for Interferon and Cytokine Research 21, 10471053, doi:10.1089/107999001317205178 (2001).

112 Whittington, H. A., Armstrong, L., Uppington, K. M. \& Millar, A. B. Interleukin-22: a potential immunomodulatory molecule in the lung. American journal of respiratory cell and molecular biology 31, 220-226, doi:10.1165/rcmb.2003-0285OC (2004).

113 Dumoutier, L., Louahed, J. \& Renauld, J. C. Cloning and characterization of IL-10-related T cell-derived inducible factor (IL-TIF), a novel cytokine structurally related to IL-10 and inducible by IL-9. J Immunol 164, 1814-1819 (2000).

114 Xie, M. H. et al. Interleukin (IL)-22, a novel human cytokine that signals through the interferon receptor-related proteins CRF2-4 and IL-22R. J Biol Chem 275, 31335-31339, doi:DOI 10.1074/jbc.M005304200 (2000).

115 Dumoutier, L., Van Roost, E., Colau, D. \& Renauld, J. C. Human interleukin-10-related T cell-derived inducible factor: Molecular cloning and functional characterization as an hepatocyte-stimulating factor. P Natl Acad Sci USA 97, 10144-10149, doi:DOI 10.1073/pnas.170291697 (2000).

116 Nagem, R. A. P. et al. Crystal structure of recombinant human interleukin-22. Structure 10, 1051-1062, doi:Doi 10.1016/S0969-2126(02)00797-9 (2002).

117 Ouyang, W., Rutz, S., Crellin, N. K., Valdez, P. A. \& Hymowitz, S. G. Regulation and functions of the IL-10 family of cytokines in inflammation and disease. Annual review of immunology 29, 71-109, doi:10.1146/annurev-immunol-031210-101312 (2011).

118 Kong, X. et al. Interleukin-22 induces hepatic stellate cell senescence and restricts liver fibrosis in mice. Hepatology 56, 1150-1159, doi:10.1002/hep.25744 (2012).

119 Andoh, A. et al. Interleukin-22, a member of the IL-10 subfamily, induces inflammatory responses in colonic subepithelial myofibroblasts. Gastroenterology 129, 969-984, doi:10.1053/j.gastro.2005.06.071 (2005).

120 Chang, Y. et al. Th17-associated cytokines promote human airway smooth muscle cell proliferation. FASEB journal : official publication of the Federation of American Societies for Experimental Biology 26, 5152-5160, doi:10.1096/fj.12-208033 (2012).

121 Dudakov, J. A. et al. Interleukin-22 drives endogenous thymic regeneration in mice. Science 336, 91-95, doi:10.1126/science.1218004 (2012).

122 Kotenko, S. V. et al. Identification of the functional interleukin-22 (IL-22) receptor complex - The IL-10R2 chain (IL-10R beta) is a common chain of both the IL-10 and IL22 (IL-10-related T cell-derived inducible factor, IL-TIF) receptor complexes. J Biol Chem 276, 2725-2732, doi:DOI 10.1074/jbc.M007837200 (2001).

123 Brand, S. et al. IL-22-mediated liver cell regeneration is abrogated by SOCS-1/3 overexpression in vitro. American journal of physiology. Gastrointestinal and liver physiology 292, G1019-1028, doi:10.1152/ajpgi.00239.2006 (2007).

124 Sonnenberg, G. F., Fouser, L. A. \& Artis, D. Border patrol: regulation of immunity, inflammation and tissue homeostasis at barrier surfaces by IL-22. Nature immunology 12, 383-390, doi:10.1038/ni.2025 (2011).

125 Lejeune, D. et al. Interleukin-22 (IL-22) activates the JAK/STAT, ERK, JNK, and p38 MAP kinase pathways in a rat hepatoma cell line. Pathways that are shared with and distinct from IL-10. J Biol Chem 277, 33676-33682, doi:10.1074/jbc.M204204200 (2002).

126 Dumoutier, L., de Meester, C., Tavernier, J. \& Renauld, J. C. New activation modus of STAT3: a tyrosine-less region of the interleukin-22 receptor recruits STAT3 by interacting 
with its coiled-coil domain. J Biol Chem 284, 26377-26384, doi:10.1074/jbc.M109.007955 (2009).

127 Meng, S. et al. Association of Shp2 with phosphorylated IL-22R1 is required for interleukin-22-induced MAP kinase activation. Journal of molecular cell biology 2, 223230, doi:10.1093/jmcb/mjq017 (2010).

128 Chung, Y. et al. Critical regulation of early Th17 cell differentiation by interleukin-1 signaling. Immunity 30, 576-587, doi:10.1016/j.immuni.2009.02.007 (2009).

129 Nurieva, R. et al. Essential autocrine regulation by IL-21 in the generation of inflammatory T cells. Nature 448, 480-483, doi:10.1038/nature05969 (2007).

130 Zhang, F., Shang, D., Zhang, Y. \& Tian, Y. Interleukin-22 suppresses the growth of A498 renal cell carcinoma cells via regulation of STAT1 pathway. PloS one 6, e20382, doi:10.1371/journal.pone.0020382 (2011).

131 Wolk, K. et al. IL-22 induces lipopolysaccharide-binding protein in hepatocytes: a potential systemic role of IL-22 in Crohn's disease. J Immunol 178, 5973-5981 (2007).

132 Ziesche, E., Bachmann, M., Kleinert, H., Pfeilschifter, J. \& Muhl, H. The interleukin22/STAT3 pathway potentiates expression of inducible nitric-oxide synthase in human colon carcinoma cells. J Biol Chem 282, 16006-16015, doi:10.1074/jbc.M611040200 (2007).

$133 \mathrm{Xu}, \mathrm{M}$. J. et al. IL-22 Ameliorates Renal Ischemia-Reperfusion Injury by Targeting Proximal Tubule Epithelium. Journal of the American Society of Nephrology : JASN, doi:10.1681/ASN.2013060611 (2014).

134 Zhang, W. et al. The pro-inflammatory cytokine IL-22 up-regulates keratin 17 expression in keratinocytes via STAT3 and ERK1/2. PloS one 7, e40797, doi:10.1371/journal.pone.0040797 (2012).

135 Ren, X., Hu, B. \& Colletti, L. M. IL-22 is involved in liver regeneration after hepatectomy. American journal of physiology. Gastrointestinal and liver physiology 298, G74-80, doi:10.1152/ajpgi.00075.2009 (2010).

136 Weber, G. F. et al. IL-22-mediated tumor growth reduction correlates with inhibition of ERK1/2 and AKT phosphorylation and induction of cell cycle arrest in the G2-M phase. $J$ Immunol 177, 8266-8272 (2006).

137 Mitra, A., Raychaudhuri, S. K. \& Raychaudhuri, S. P. IL-22 induced cell proliferation is regulated by $\mathrm{PI} 3 \mathrm{~K} / \mathrm{Akt} / \mathrm{mTOR}$ signaling cascade. Cytokine 60, 38-42, doi:10.1016/j.cyto.2012.06.316 (2012).

138 Fukui, H. et al. DMBT1 is a novel gene induced by IL-22 in ulcerative colitis. Inflammatory bowel diseases 17, 1177-1188, doi:10.1002/ibd.21473 (2011).

139 Cho, K. A. et al. Interleukin-17 and Interleukin-22 Induced Proinflammatory Cytokine Production in Keratinocytes via Inhibitor of Nuclear Factor kappaB Kinase-alpha Expression. Annals of dermatology 24, 398-405, doi:10.5021/ad.2012.24.4.398 (2012).

140 Grivennikov, S. I. \& Karin, M. Dangerous liaisons: STAT3 and NF-kappaB collaboration and crosstalk in cancer. Cytokine \& growth factor reviews 21, 11-19, doi:10.1016/j.cytogfr.2009.11.005 (2010).

141 Yu, H., Pardoll, D. \& Jove, R. STATs in cancer inflammation and immunity: a leading role for STAT3. Nature reviews. Cancer 9, 798-809, doi:10.1038/nrc2734 (2009).

$142 \mathrm{Sa}, \mathrm{S}$. M. et al. The effects of IL-20 subfamily cytokines on reconstituted human epidermis suggest potential roles in cutaneous innate defense and pathogenic adaptive immunity in psoriasis. J Immunol 178, 2229-2240 (2007). 
143 Nograles, K. E. et al. Th17 cytokines interleukin (IL)-17 and IL-22 modulate distinct inflammatory and keratinocyte-response pathways. The British journal of dermatology 159, 1092-1102, doi:10.1111/j.1365-2133.2008.08769.x (2008).

144 Cho, K. A., Suh, J. W., Lee, K. H., Kang, J. L. \& Woo, S. Y. IL-17 and IL-22 enhance skin inflammation by stimulating the secretion of IL-1beta by keratinocytes via the ROSNLRP3-caspase-1 pathway. International immunology 24, 147-158, doi:10.1093/intimm/dxr110 (2012).

145 Bansal, G. et al. IL-22 activates oxidant signaling in pulmonary vascular smooth muscle cells. Cellular signalling 25, 2727-2733, doi:10.1016/j.cellsig.2013.09.001 (2013).

146 Murano, T. et al. Hes1 promotes the IL-22-mediated antimicrobial response by enhancing STAT3-dependent transcription in human intestinal epithelial cells. Biochemical and biophysical research communications, doi:10.1016/j.bbrc.2013.12.061 (2013).

147 Guilloteau, K. et al. Skin Inflammation Induced by the Synergistic Action of IL-17A, IL22, Oncostatin M, IL-1 $\{$ alpha\}, and TNF-\{alpha\} Recapitulates Some Features of Psoriasis. J Immunol, doi:10.4049/jimmunol.0902464 (2010).

148 Ma, H. L. et al. IL-22 is required for Th17 cell-mediated pathology in a mouse model of psoriasis-like skin inflammation. The Journal of clinical investigation 118, 597-607, doi:10.1172/JCI33263 (2008).

149 Boniface, K. et al. A role for T cell-derived interleukin 22 in psoriatic skin inflammation. Clinical and experimental immunology 150, 407-415, doi:10.1111/j.13652249.2007.03511.x (2007).

150 Pennino, D. et al. IL-22 suppresses IFN-gamma-mediated lung inflammation in asthmatic patients. The Journal of allergy and clinical immunology 131, 562-570, doi:10.1016/j.jaci.2012.09.036 (2013).

151 Pham, T. A. et al. Epithelial IL-22RA1-mediated fucosylation promotes intestinal colonization resistance to an opportunistic pathogen. Cell host \& microbe 16, 504-516, doi:10.1016/j.chom.2014.08.017 (2014).

152 Sano, T. et al. An IL-23R/IL-22 Circuit Regulates Epithelial Serum Amyloid A to Promote Local Effector Th17 Responses. Cell 163, 381-393, doi:10.1016/j.cell.2015.08.061 (2015).

153 Armitage, A. E. et al. Hepcidin regulation by innate immune and infectious stimuli. Blood 118, 4129-4139, doi:10.1182/blood-2011-04-351957 (2011).

154 Smith, C. L. et al. IL-22 regulates iron availability in vivo through the induction of hepcidin. Journal of immunology 191, 1845-1855, doi:10.4049/jimmunol.1202716 (2013).

155 Yang, L. et al. Amelioration of high fat diet induced liver lipogenesis and hepatic steatosis by interleukin-22. Journal of hepatology 53, 339-347, doi:10.1016/j.jhep.2010.03.004 (2010).

156 Sonnenberg, G. F. et al. Pathological versus protective functions of IL-22 in airway inflammation are regulated by IL-17A. $J$ Exp Med 207, 1293-1305, doi:10.1084/jem.20092054 (2010).

$157 \mathrm{Xu}, \mathrm{W}$. et al. A soluble class II cytokine receptor, IL-22RA2, is a naturally occurring IL22 antagonist. Proc Natl Acad Sci U S A 98, 9511-9516, doi:10.1073/pnas.171303198 (2001).

158 Kotenko, S. V. et al. Identification, cloning, and characterization of a novel soluble receptor that binds IL-22 and neutralizes its activity. J Immunol 166, 7096-7103 (2001).

159 Weiss, B. et al. Cloning of murine IL-22 receptor alpha 2 and comparison with its human counterpart. Genes and immunity 5, 330-336, doi:10.1038/sj.gene.6364104 (2004). 
160 Wei, C. C., Ho, T. W., Liang, W. G., Chen, G. Y. \& Chang, M. S. Cloning and characterization of mouse IL-22 binding protein. Genes and immunity 4, 204-211, doi:10.1038/sj.gene.6363947 (2003).

161 Martin, J. C. et al. Interleukin-22 binding protein (IL-22BP) is constitutively expressed by a subset of conventional dendritic cells and is strongly induced by retinoic acid. Mucosal immunology 7, 101-113, doi:10.1038/mi.2013.28 (2014).

162 Jiang, R. et al. Interleukin-22 promotes human hepatocellular carcinoma by activation of STAT3. Hepatology 54, 900-909, doi:10.1002/hep.24486 (2011).

163 Wade, R. et al. Association between single nucleotide polymorphism-genotype and outcome of patients with chronic lymphocytic leukemia in a randomized chemotherapy trial. Haematologica 96, 1496-1503, doi:10.3324/haematol.2011.043471 (2011).

164 Beyeen, A. D. et al. IL-22RA2 associates with multiple sclerosis and macrophage effector mechanisms in experimental neuroinflammation. $J$ Immunol 185, 6883-6890, doi:10.4049/jimmunol.1001392 (2010).

165 Reimann, E. et al. The mRNA expression profile of cytokines connected to the regulation of melanocyte functioning in vitiligo skin biopsy samples and peripheral blood mononuclear cells. Human immunology 73, 393-398, doi:10.1016/j.humimm.2012.01.011 (2012).

166 Sertorio, M. et al. IL-22 and IL-22 binding protein (IL-22BP) regulate fibrosis and cirrhosis in hepatitis $\mathrm{C}$ virus and schistosome infections. Hepatology 61, 1321-1331, doi:10.1002/hep.27629 (2015).

167 Perriard, G. et al. Interleukin-22 is increased in multiple sclerosis patients and targets astrocytes. Journal of neuroinflammation 12, 119, doi:10.1186/s12974-015-0335-3 (2015).

168 Yang, X. et al. Increased urinary interleukin 22 binding protein levels correlate with lupus nephritis activity. The Journal of rheumatology 41, 1793-1800, doi:10.3899/jrheum.131292 (2014).

169 Jones, B. C., Logsdon, N. J. \& Walter, M. R. Structure of IL-22 bound to its high-affinity IL-22R1 chain. Structure 16, 1333-1344, doi:10.1016/j.str.2008.06.005 (2008).

170 Piriyapongsa, J., Polavarapu, N., Borodovsky, M. \& McDonald, J. Exonization of the LTR transposable elements in human genome. BMC genomics 8, 291, doi:10.1186/1471-21648-291 (2007).

171 Dumoutier, L., Leemans, C., Lejeune, D., Kotenko, S. V. \& Renauld, J. C. Cutting edge: STAT activation by IL-19, IL-20 and mda-7 through IL-20 receptor complexes of two types. J Immunol 167, 3545-3549 (2001).

172 Chung, Y. et al. Expression and regulation of IL-22 in the IL-17-producing CD4+ T lymphocytes. Cell research 16, 902-907, doi:10.1038/sj.cr.7310106 (2006).

173 Guo, Y. et al. Intracellular overexpression of HIV-1 Nef impairs differentiation and maturation of monocytic precursors towards dendritic cells. PloS one 7, e40179, doi:10.1371/journal.pone.0040179 (2012).

174 Berges, C. et al. A cell line model for the differentiation of human dendritic cells. Biochemical and biophysical research communications 333, 896-907, doi:10.1016/j.bbrc.2005.05.171 (2005).

175 Manicassamy, S. et al. Toll-like receptor 2-dependent induction of vitamin A-metabolizing enzymes in dendritic cells promotes $\mathrm{T}$ regulatory responses and inhibits autoimmunity. Nature medicine 15, 401-409, doi:10.1038/nm.1925 (2009). 
176 Manoharan, I. et al. TLR2-dependent activation of beta-catenin pathway in dendritic cells induces regulatory responses and attenuates autoimmune inflammation. J Immunol 193, 4203-4213, doi:10.4049/jimmunol.1400614 (2014).

177 Geissmann, F. et al. Retinoids regulate survival and antigen presentation by immature dendritic cells. J Exp Med 198, 623-634, doi:10.1084/jem.20030390 (2003).

178 Yost, C. C. et al. Activated polymorphonuclear leukocytes rapidly synthesize retinoic acid receptor-alpha: a mechanism for translational control of transcriptional events. J Exp Med 200, 671-680, doi:10.1084/jem.20040224 (2004).

179 Zeng, R. et al. Retinoic acid regulates the development of a gut-homing precursor for intestinal dendritic cells. Mucosal immunology 6, 847-856, doi:10.1038/mi.2012.123 (2013).

180 Martin, S. J., Bradley, J. G. \& Cotter, T. G. HL-60 cells induced to differentiate towards neutrophils subsequently die via apoptosis. Clinical and experimental immunology 79, 448-453 (1990).

181 Carrigan, S. O., Weppler, A. L., Issekutz, A. C. \& Stadnyk, A. W. Neutrophil differentiated HL-60 cells model Mac-1 (CD11b/CD18)-independent neutrophil transepithelial migration. Immunology 115, 108-117, doi:10.1111/j.1365-2567.2005.02131.x (2005).

182 Jacob, C. et al. DMSO-treated HL60 cells: a model of neutrophil-like cells mainly expressing PDE4B subtype. International immunopharmacology 2, 1647-1656 (2002).

183 Logsdon, N. J. et al. The IL-10R2 binding hot spot on IL-22 is located on the N-terminal helix and is dependent on N-linked glycosylation. Journal of molecular biology 342, 503514, doi:10.1016/j.jmb.2004.07.069 (2004).

184 Wu, P. W. et al. IL-22R, IL-10R2, and IL-22BP binding sites are topologically juxtaposed on adjacent and overlapping surfaces of IL-22. Journal of molecular biology 382, 11681183, doi:10.1016/j.jmb.2008.07.046 (2008).

185 Gruenberg, B. H. et al. A novel, soluble homologue of the human IL-10 receptor with preferential expression in placenta. Genes and immunity 2, 329-334, doi:10.1038/sj.gene.6363786 (2001).

186 Lill, C. M. et al. Assessment of microRNA-related SNP effects in the 3' untranslated region of the IL22RA2 risk locus in multiple sclerosis. Neurogenetics 15, 129-134, doi:10.1007/s10048-014-0396-y (2014).

187 Kotenko, S. V. et al. IFN-lambdas mediate antiviral protection through a distinct class II cytokine receptor complex. Nature immunology 4, 69-77, doi:10.1038/ni875 (2003).

188 Dellgren, C., Gad, H. H., Hamming, O. J., Melchjorsen, J. \& Hartmann, R. Human interferon-lambda3 is a potent member of the type III interferon family. Genes and immunity 10, 125-131, doi:10.1038/gene.2008.87 (2009).

189 Bolen, C. R., Ding, S., Robek, M. D. \& Kleinstein, S. H. Dynamic expression profiling of type I and type III interferon-stimulated hepatocytes reveals a stable hierarchy of gene expression. Hepatology 59, 1262-1272, doi:10.1002/hep.26657 (2014).

190 Sheppard, P. et al. IL-28, IL-29 and their class II cytokine receptor IL-28R. Nature immunology 4, 63-68, doi:10.1038/ni873 (2003).

191 Kotenko, S. V. Vol. 23 583-590 (2011).

192 Marcello, T. et al. Interferons alpha and lambda inhibit hepatitis C virus replication with distinct signal transduction and gene regulation kinetics. Gastroenterology 131, 18871898, doi:10.1053/j.gastro.2006.09.052 (2006). 
193 Voigt, E. A. \& Yin, J. Kinetic Differences and Synergistic Antiviral Effects Between Type I and Type III Interferon Signaling Indicate Pathway Independence. Journal of interferon \& cytokine research : the official journal of the International Society for Interferon and Cytokine Research 35, 734-747, doi:10.1089/jir.2015.0008 (2015).

194 Freeman, J. et al. Pegylated interferons Lambda-1a and alfa-2a display different gene induction and cytokine and chemokine release profiles in whole blood, human hepatocytes and peripheral blood mononuclear cells. Journal of viral hepatitis 21, e1-9, doi:10.1111/jvh.12243 (2014).

195 Maher, S. G. et al. IFNalpha and IFNlambda differ in their antiproliferative effects and duration of JAK/STAT signaling activity. Cancer biology \& therapy 7, 1109-1115 (2008).

196 Dumoutier, L. et al. Role of the interleukin (IL)-28 receptor tyrosine residues for antiviral and antiproliferative activity of IL-29/interferon-lambda 1: similarities with type I interferon signaling. J Biol Chem 279, 32269-32274, doi:10.1074/jbc.M404789200 (2004).

197 Doyle, S. E. et al. Interleukin-29 uses a type 1 interferon-like program to promote antiviral responses in human hepatocytes. Hepatology 44, 896-906, doi:10.1002/hep.21312 (2006).

198 Zhou, Z. et al. Type III interferon (IFN) induces a type I IFN-like response in a restricted subset of cells through signaling pathways involving both the Jak-STAT pathway and the mitogen-activated protein kinases. J Virol 81, 7749-7758, doi:10.1128/JVI.02438-06 (2007).

199 Kohli, A. et al. Distinct and overlapping genomic profiles and antiviral effects of Interferon-lambda and -alpha on HCV-infected and noninfected hepatoma cells. Journal of viral hepatitis 19, 843-853, doi:10.1111/j.1365-2893.2012.01610.x (2012).

200 Odendall, C. et al. Diverse intracellular pathogens activate type III interferon expression from peroxisomes. Nature immunology 15, 717-726, doi:10.1038/ni.2915 (2014).

201 Bender, S. et al. Activation of Type I and III Interferon Response by Mitochondrial and Peroxisomal MAVS and Inhibition by Hepatitis C Virus. PLoS Pathog 11, e1005264, doi:10.1371/journal.ppat.1005264 (2015).

202 Mordstein, M. et al. Interferon-lambda contributes to innate immunity of mice against influenza A virus but not against hepatotropic viruses. PLoS Pathog 4, e1000151, doi:10.1371/journal.ppat.1000151 (2008).

203 Mordstein, M. et al. Lambda interferon renders epithelial cells of the respiratory and gastrointestinal tracts resistant to viral infections. Journal of virology 84, 5670-5677, doi:10.1128/JVI.00272-10 (2010).

204 Crotta, S. et al. Type I and type III interferons drive redundant amplification loops to induce a transcriptional signature in influenza-infected airway epithelia. PLoS Pathog 9, e1003773, doi:10.1371/journal.ppat.1003773 (2013).

205 Jewell, N. a. et al. Lambda interferon is the predominant interferon induced by influenza a virus infection in vivo. Journal of virology 84, 11515-11522, doi:10.1128/JVI.01703-09 (2010).

206 Contoli, M. et al. Role of deficient type III interferon-lambda production in asthma exacerbations. Nature medicine 12, 1023-1026, doi:10.1038/nm1462 (2006).

207 Pott, J. et al. IFN-\{lambda $\}$ determines the intestinal epithelial antiviral host defense. $P$ Natl Acad Sci USA 108, 7944-7949, doi:10.1073/pnas.1100552108 (2011).

208 Mahlakoiv, T., Hernandez, P., Gronke, K., Diefenbach, A. \& Staeheli, P. Leukocytederived IFN-alpha/beta and epithelial IFN-lambda constitute a compartmentalized mucosal 
defense system that restricts enteric virus infections. PLoS Pathog 11, e1004782, doi:10.1371/journal.ppat.1004782 (2015).

209 Nice, T. J. et al. Interferon-lambda cures persistent murine norovirus infection in the absence of adaptive immunity. Science 347, 269-273, doi:10.1126/science.1258100 (2015).

210 Wolk, K. et al. IL-29 is produced by $\mathrm{T}(\mathrm{H}) 17$ cells and mediates the cutaneous antiviral competence in psoriasis. Science translational medicine 5, 204ra129, doi:10.1126/scitranslmed.3006245 (2013).

$211 \mathrm{Ge}, \mathrm{D}$. et al. Genetic variation in IL28B predicts hepatitis C treatment-induced viral clearance. Nature 461, 399-401, doi:10.1038/nature08309 (2009).

212 Rauch, A. et al. Genetic Variation in IL28B Is Associated With Chronic Hepatitis C and Treatment Failure: A Genome-Wide Association Study. Gastroenterology 138, 13381345, 1345.e1331-1337, doi:10.1053/j.gastro.2009.12.056 (2010).

213 Suppiah, V.et al. IL28B is associated with response to chronic hepatitis C interferon-alpha and ribavirin therapy. Nature genetics 41, 1100-1104, doi:10.1038/ng.447 (2009).

214 Tanaka, Y. et al. Genome-wide association of IL28B with response to pegylated interferonalpha and ribavirin therapy for chronic hepatitis C. Nat Genet 41, 1105-1109, doi:10.1038/ng.449 (2009).

215 Thomas, D. L. et al. Genetic variation in IL28B and spontaneous clearance of hepatitis C virus. Nature 461, 798-801, doi:10.1038/nature08463 (2009).

216 Prokunina-Olsson, L. et al. A variant upstream of IFNL3 (IL28B) creating a new interferon gene IFNL4 is associated with impaired clearance of hepatitis C virus. Nature genetics $\mathbf{4 5}$, 164-171, doi:10.1038/ng.2521 (2013).

217 McFarland, A. P. et al. The favorable IFNL3 genotype escapes mRNA decay mediated by AU-rich elements and hepatitis C virus-induced microRNAs. Nature immunology 15, 7279, doi:10.1038/ni.2758 (2014).

$218 \mathrm{Lu}, \mathrm{Y}$. F. et al. IFNL3 mRNA structure is remodeled by a functional non-coding polymorphism associated with hepatitis C virus clearance. Sci Rep 5, 16037, doi:10.1038/srep16037 (2015).

219 Lu, Y. F., Goldstein, D. B., Urban, T. J. \& Bradrick, S. S. Interferon-lambda4 is a cellautonomous type III interferon associated with pre-treatment hepatitis $\mathrm{C}$ virus burden. Virology 476, 334-340, doi:10.1016/j.virol.2014.12.020 (2015).

220 O'Brien, T. R. et al. Comparison of functional variants in IFNL4 and IFNL3 for association with HCV clearance. J Hepatol 63, 1103-1110, doi:10.1016/j.jhep.2015.06.035 (2015).

221 Peiffer, K. H. et al. IFN lambda 4 genotypes and resistance-associated variants in HCV genotype 1 and 3 infected patients. Hepatology, doi:10.1002/hep.28255 (2015).

222 Aka, P. V. et al. Association of the IFNL4-DeltaG Allele With Impaired Spontaneous Clearance of Hepatitis C Virus. J Infect Dis 209, 350-354, doi:10.1093/infdis/jit433 (2014).

223 Meissner, E. G. et al. IFNL4-DeltaG genotype is associated with slower viral clearance in hepatitis C, genotype-1 patients treated with sofosbuvir and ribavirin. J Infect Dis 209, 1700-1704, doi:10.1093/infdis/jit827 (2014).

224 Hamming, O. J. et al. Interferon lambda 4 signals via the IFN $\lambda$ receptor to regulate antiviral activity against $\mathrm{HCV}$ and coronaviruses. The EMBO journal 32, 3055-3065, doi:10.1038/emboj.2013.232 (2013).

225 Onabajo, O. O. et al. Expression of Interferon Lambda 4 Is Associated with Reduced Proliferation and Increased Cell Death in Human Hepatic Cells. Journal of interferon \& 
cytokine research : the official journal of the International Society for Interferon and Cytokine Research 35, 888-900, doi:10.1089/jir.2014.0161 (2015).

226 Noureddin, M. et al. Hepatic expression levels of interferons and interferon-stimulated genes in patients with chronic hepatitis C: A phenotype-genotype correlation study. Genes and immunity 16, 321-329, doi:10.1038/gene.2015.11 (2015).

227 Amanzada, A., Kopp, W., Spengler, U., Ramadori, G. \& Mihm, S. Interferon-lambda4 (IFNL4) transcript expression in human liver tissue samples. PloS one 8, e84026, doi:10.1371/journal.pone.0084026 (2013).

228 Terczynska-Dyla, E. et al. Reduced IFNlambda4 activity is associated with improved HCV clearance and reduced expression of interferon-stimulated genes. Nature communications 5, 5699, doi:10.1038/ncomms6699 (2014).

229 Booth, D. \& George, J. Loss of function of the new interferon IFN-lambda4 may confer protection from hepatitis C. Nat Genet 45, 119-120, doi:10.1038/ng.2537 (2013).

230 McBride, D. Genetic variant links hepatitis $\mathrm{C}$ virus clearance and treatment response. ONS Connect 28, 50 (2013).

231 Ray, K. Hepatitis: New gene IFNL4 is associated with impaired clearance of HCV. Nat Rev Gastroenterol Hepatol 10, 63, doi:10.1038/nrgastro.2013.7 (2013).

232 Bibert, S. et al. IL28B expression depends on a novel TT/-G polymorphism which improves HCV clearance prediction. The Journal of experimental medicine 210, 11091116, doi:10.1084/jem.20130012 (2013).

233 Liu, S. et al. Measuring antiviral activity of benzimidazole molecules that alter IRES RNA structure with an infectious hepatitis C virus chimera expressing Renilla luciferase. Antiviral Res 89, 54-63, doi:10.1016/j.antiviral.2010.11.004 (2011).

234 Stone, A. E. et al. Hepatitis C virus pathogen associated molecular pattern (PAMP) triggers production of lambda-interferons by human plasmacytoid dendritic cells. PLoS Pathog 9, e1003316, doi:10.1371/journal.ppat.1003316 (2013).

235 Coccia, E. M. et al. Viral infection and Toll-like receptor agonists induce a differential expression of type I and lambda interferons in human plasmacytoid and monocyte-derived dendritic cells. European journal of immunology 34, 796-805, doi:10.1002/eji.200324610 (2004).

236 Proudfoot, N. J. Ending the message: poly(A) signals then and now. Genes \& Development 25, 1770-1782, doi:10.1101/gad.17268411 (2011).

237 Keller, B. C. et al. Resistance to alpha/beta interferon is a determinant of West Nile virus replication fitness and virulence. J Virol 80, 9424-9434, doi:10.1128/JVI.00768-06 (2006).

238 Suthar, M. S. et al. IPS-1 is essential for the control of West Nile virus infection and immunity. PLoS Pathog 6, e1000757, doi:10.1371/journal.ppat.1000757 (2010). 


\section{VITA}

Chrissie was born and raised in Singapore; there she obtained her education up to GCE "A" Levels. As an undergraduate, she attended University College London in the United Kingdom, where she earned a Bachelor of Science in Immunology in 2010. She completed her undergraduate dissertation with Dr. Elizabeth Jury at UCL's Centre for Rheumatology Research, where she examined the correlation between membrane lipid orders of $\mathrm{T}$ and $\mathrm{B}$ lymphocytes and their polarization or functional phenotypes, as well as correlation with the serum lipids of rheumatic disease patients. She then joined the lab of Dr. Jean-Pierre Abastado at the Singapore Immunology Network, where she studied the T cell polarization signature in gastric tumors through 2010-2011. She enrolled in the Immunology program at the University of Washington in Fall 2011 and permanently joined the laboratory of Dr. Ram Savan in Summer 2012. Her undergraduate and graduate studies were both supported by the Agency for Science, Technology and Research (A*STAR) in Singapore including full tuition, stipend and conference travel. 